DFPD-04/TH/06

\title{
Anatomy and Phenomenology of $\mu-\tau$ Lepton Flavour Violation in the MSSM
}

\author{
Andrea Brignole ${ }^{1}$ and Anna Rossi ${ }^{2}$ \\ Dipartimento di Fisica 'G. Galilei', Università di Padova and \\ INFN, Sezione di Padova, Via Marzolo 8, I-35131 Padua, Italy
}

\begin{abstract}
We perform a detailed analysis of several $\mu-\tau$ lepton flavour violating (LFV) processes, namely $\tau \rightarrow \mu X\left(X=\gamma, e^{+} e^{-}, \mu^{+} \mu^{-}, \rho, \pi, \eta, \eta^{\prime}\right), Z \rightarrow \mu \tau$ and Higgs boson decays into $\mu \tau$. First we present a model independent operator analysis relevant to such decays, then we explicitly compute the LFV operator coefficients [and $\left(g_{\mu}-2\right)$ ] in a general unconstrained MSSM framework, allowing slepton mass matrices to have large $\mu-\tau$ entries. We systematically study the role and the interplay of dipole and non-dipole operators, showing how the rates and the mutual correlations of those LFV decays change in different regions of the MSSM parameter space. Values of the LFV branching ratios in the experimentally interesting range $10^{-9}-10^{-7}$ can be achieved. For at least two MSSM Higgs bosons, the branching ratio of the LFV decay into $\mu \tau$ can reach values of order $10^{-4}$.
\end{abstract}

\footnotetext{
${ }^{1}$ E-mail address: andrea.brignole@pd.infn.it

${ }^{2}$ E-mail address: anna.rossi@pd.infn.it
} 


\section{Introduction}

The search for lepton flavour violating processes is an important window into physics beyond the Standard Model. In the Minimal Supersymmetric extension of the Standard Model (MSSM) several such processes can have non-negligible rates, provided the mass matrices of the leptons and of the sleptons are not aligned. The experimental bounds strongly constrain the amount of misalignment in the $e-\mu$ sector, whilst the constraints are weaker for the $\mu-\tau$ or $e-\tau$ sector (see $e . g$. $[1,2])$. In this work we thoroughly explore the case of large $\mu-\tau$ lepton flavour violation (LFV) in a general unconstrained MSSM framework, assuming negligible LFV in the other sectors. The case of large $\mu-\tau$ LFV is partly motivated also by the observed atmospheric neutrino anomaly, which can be interpreted in terms of $\nu_{\mu} \rightarrow \nu_{\tau}$ oscillations with maximal mixing angle [3]. The prototype and most studied LFV process involving $\mu$ and $\tau$ is the radiative decay $\tau \rightarrow \mu \gamma$, which proceeds via dipole operators. Besides this decay, we also consider other interesting LFV processes, such as $\tau \rightarrow \mu e e, \tau \rightarrow \mu \mu \mu, \tau \rightarrow \mu \rho, \tau \rightarrow \mu \pi, \tau \rightarrow \mu \eta, \tau \rightarrow \mu \eta^{\prime}, Z \rightarrow \mu \tau$, which may have different degrees of correlation with $\tau \rightarrow \mu \gamma$. The present experimental bounds on all these decays are:

$$
\begin{aligned}
B R\left(\tau^{-} \rightarrow \mu^{-} \gamma\right) & <3.1 \times 10^{-7}[4], \\
B R\left(\tau^{-} \rightarrow \mu^{-} e^{+} e^{-}\right) & <1.9 \times 10^{-7}[5], \\
B R\left(\tau^{-} \rightarrow \mu^{-} \mu^{+} \mu^{-}\right) & <1.9 \times 10^{-7}[6,5], \\
B R\left(\tau^{-} \rightarrow \mu^{-} \rho^{0}\right) & <6.3 \times 10^{-6}[7], \\
B R\left(\tau^{-} \rightarrow \mu^{-} \pi^{0}\right) & <4.0 \times 10^{-6}[7], \\
B R\left(\tau^{-} \rightarrow \mu^{-} \eta\right) & <3.4 \times 10^{-7} \\
B R\left(Z \rightarrow \mu^{+} \tau^{-}\right) & <1.2 \times 10^{-5}
\end{aligned}
$$

(We are not aware of experimental bounds on $\tau \rightarrow \mu \eta^{\prime}$.) In the MSSM with large $\mu-\tau$ flavour violation, a combined analysis of all such processes is very instructive, especially in view of the future sensitivity on those branching ratios, which may reach $10^{-8}-10^{-9}[9,10]$. In particular, it is interesting to study the interplay between dipole and non-dipole operators. In addition, we consider the LFV decays of MSSM Higgs bosons into $\mu \tau$ [11], which are correlated to some of the above processes and can give testable signatures at future colliders.

The outline of this paper is as follows. In Section 2 we introduce the relevant effective operators and compute the branching ratios of the LFV decays. In Section 3 we focus on the MSSM, exhibit the sources of LFV in the left and right slepton mass matrices and discuss the computation of the operator coefficients [including the Higgs- $\mu-\tau$ and $\left(g_{\mu}-2\right)$ ones], whose analytical expressions are displayed in Appendix. In Section 4 we give a numerical discussion of the branching ratios in the case of large LFV in the left sector. In Section 5 we present an analogous discussion for large LFV in the right sector. Finally, in Section 6 we summarize our results. 


\section{Effective operators and branching ratios}

This section is devoted to the 'model-independent' calculation of the LFV branching ratios. Namely, first we parametrize the basic effective operators (Section 2.1), then construct appropriate effective lagrangians and compute the branching ratios in terms of the coefficients of the effective operators (Section 2.2). We also discuss correlations among different processes in cases of single-operator-dominance (Section 2.3).

\subsection{Parametrization of LFV effective operators}

Our first step, in the derivation of the LFV branching ratios, is the parametrization of the LFV operators that contain a muon, a tau and either a $Z$ boson, or a photon, or an additional $f$-fermion pair. The leading contributions to these operators arise from $d=6 S U(2)_{W} \times$ $U(1)_{Y}$-invariant operators [12], possibly with Higgs fields set at their vacuum expectation values (VEVs). It is useful to keep this in mind, although we will not write explicitly the operators in the unbroken phase (except for a few examples). We also recall that the MSSM contains two Higgs doublets $H_{1}, H_{2}$, whose VEVs define the ratio $\tan \beta=\left\langle H_{2}^{0}\right\rangle /\left\langle H_{1}^{0}\right\rangle$. We postpone the discussion of $d=4$ Higgs-muon-tau effective operators to Section 3.2. The parametrization given below assumes fermions to be on-shell, whereas gauge bosons could also be off-shell. We keep the tau mass $m_{\tau}$ at the leading order and neglect $m_{\mu}, m_{e}$ as well as the light-quark masses. We adopt two-component spinor notation, so for example $\mu$ and $\tau\left(\bar{\mu}^{c}\right.$ and $\left.\bar{\tau}^{c}\right)$ are the left-handed (right-handed) components of the muon and tau fields, respectively ${ }^{1}$. Sometimes (e.g. in Figs. 1, 2, 4) symbols like $\mu, \tau, f$ will generically refer to the particles, not to specific chiralities. Finally, it is understood that the coefficients of all the LFV operators below should carry a flavour subscript $\mu \tau$, which we omit for brevity.

\section{$\tau \mu Z$ effective operators}

We distinguish the following operators:

$$
\begin{gathered}
g_{Z} m_{Z}^{2}\left[A_{L}^{Z} \bar{\mu} \bar{\sigma}^{\mu} \tau+A_{R}^{Z} \mu^{c} \sigma^{\mu} \bar{\tau}^{c}+\text { h.c. }\right] Z_{\mu}, \\
g_{Z}\left[C_{L}^{Z} \bar{\mu} \bar{\sigma}^{\mu} \tau+C_{R}^{Z} \mu^{c} \sigma^{\mu} \bar{\tau}^{c}+\text { h.c. }\right] \square Z_{\mu}, \\
g_{Z} m_{\tau}\left[i D_{L}^{Z} \bar{\mu} \bar{\sigma}^{\mu \nu} \bar{\tau}^{c}+i D_{R}^{Z} \mu^{c} \sigma^{\mu \nu} \tau+\text { h.c. }\right] Z_{\mu \nu},
\end{gathered}
$$

where $g_{Z}=\sqrt{g^{2}+g^{\prime 2}}\left(g, g^{\prime}\right.$ being the $S U(2)_{W}$ and $U(1)_{Y}$ coupling constants, respectively) and $Z_{\mu \nu}=\partial_{\mu} Z_{\nu}-\partial_{\nu} Z_{\mu}$. The operators in (8) are chirality conserving (monopole) and have no derivatives, so they originate from $S U(2)_{W} \times U(1)_{Y}$-invariant operators with at least two

\footnotetext{
${ }^{1}$ For instance, in terms of the four-component spinors $\psi_{\mu}^{T}=\left(\mu \bar{\mu}^{c}\right), \psi_{\tau}^{T}=\left(\tau \bar{\tau}^{c}\right)$, the bilinears $\bar{\mu} \bar{\sigma}^{\nu} \tau$ and $\mu^{c} \sigma^{\nu} \bar{\tau}^{c}$ correspond to $\bar{\psi}_{\mu} \gamma^{\nu} P_{L} \psi_{\tau}$ and $\bar{\psi}_{\mu} \gamma^{\nu} P_{R} \psi_{\tau}\left[P_{L, R}=\frac{1}{2}\left(1 \mp \gamma^{5}\right)\right]$ respectively. We take $\sigma^{\mu} \equiv(1, \boldsymbol{\sigma}), \bar{\sigma}^{\mu} \equiv$ $(1,-\boldsymbol{\sigma}), \sigma^{\mu \nu} \equiv \frac{1}{4}\left(\sigma^{\mu} \bar{\sigma}^{\nu}-\sigma^{\nu} \bar{\sigma}^{\mu}\right), \bar{\sigma}^{\mu \nu} \equiv \frac{1}{4}\left(\bar{\sigma}^{\mu} \sigma^{\nu}-\bar{\sigma}^{\nu} \sigma^{\mu}\right)$ and $g_{\mu \nu}=\operatorname{diag}(+1,-1,-1,-1)$, where $\boldsymbol{\sigma}=\left(\sigma^{1}, \sigma^{2}, \sigma^{3}\right)$ are the $2 \times 2$ Pauli matrices.
} 
Higgs fields, which reflects in the factor $m_{Z}^{2}$ we have extracted out. For instance, the leading contributions to (8) come from operators such as:

$$
\left(\bar{L}_{\mu} \bar{\sigma}^{\mu} L_{\tau}\right)\left(H_{1}^{\dagger} i D_{\mu} H_{1}\right), \quad\left(\bar{L}_{\mu} \bar{\sigma}^{\mu} \sigma^{a} L_{\tau}\right)\left(H_{1}^{\dagger} \sigma^{a} i D_{\mu} H_{1}\right), \quad\left(\mu^{c} \sigma^{\mu} \bar{\tau}^{c}\right)\left(H_{1}^{\dagger} i D_{\mu} H_{1}\right)
$$

and analogous ones with $H_{1}^{\dagger} H_{1}$ replaced by $H_{2}^{\dagger} H_{2}$ or $H_{2} H_{1}$ (+h.c.), where $L_{\mu}, L_{\tau}$ are the $S U(2)_{W}$ lepton doublets. The operators in (9) are also chirality conserving (monopole) and have two derivatives, so they originate from $S U(2)_{W} \times U(1)_{Y}$-invariant operators with no Higgs fields at the leading order. The operators in (10) are chirality flipping (dipole) and come from $S U(2)_{W} \times U(1)_{Y}$-invariant operators with at least one Higgs field, hence we have pulled out a factor $m_{\tau}$. In the case of virtual $Z$, only the $A^{Z}$-operators need to be considered since the remaining ones (and other ones not listed above) give suppressed contributions. In the case of real $Z$, the $A^{Z}$ - and $C^{Z}$-operators give contributions of the same order ${ }^{2}$ (notice that $\square \rightarrow-m_{Z}^{2}$ ). The $D^{Z}$-operators give comparable contributions if the $m_{\tau}$ suppression is compensated by a large $\tan \beta$ factor, induced by the Higgs field $H_{2}$ (notice that $50 \times m_{\tau} \sim m_{Z}$ ).

\section{$\underline{\tau \mu \gamma}$ effective operators}

We distinguish the following operators:

$$
\begin{gathered}
e\left[C_{L}^{\gamma} \bar{\mu} \bar{\sigma}^{\mu} \tau+C_{R}^{\gamma} \mu^{c} \sigma^{\mu} \bar{\tau}^{c}+\text { h.c. }\right] \square A_{\mu}, \\
e m_{\tau}\left[i D_{L}^{\gamma} \bar{\mu} \bar{\sigma}^{\mu \nu} \bar{\tau}^{c}+i D_{R}^{\gamma} \mu^{c} \sigma^{\mu \nu} \tau+\text { h.c. }\right] F_{\mu \nu} .
\end{gathered}
$$

The operators in eqs. (12),(13) are analogous to the $C^{Z}$ and $D^{Z}$ operators, respectively. We recall that the $Z$ and photon operators have a common origin in terms of $S U(2)_{W} \times U(1)_{Y^{-}}$ invariant operators. However, the $A^{Z}$-operators (8) have no photon counterpart because of the unbroken $U(1)_{e m}$ symmetry. In the case of virtual photon, both the monopole and dipole operators contribute, whereas in the case of real photon only the latter ones do.

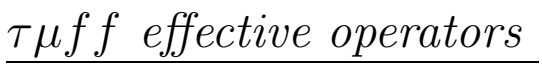

We parametrize four-fermion operators as follows ${ }^{3}$ :

$$
\sum_{f}\left[\left(\bar{\mu} \bar{\sigma}^{\mu} \tau\right)\left(B_{L}^{f_{L}} \bar{f} \bar{\sigma}_{\mu} f+B_{L}^{f_{R}} f^{c} \sigma_{\mu} \bar{f}^{c}\right)+\left(\mu^{c} \sigma^{\mu} \bar{\tau}^{c}\right)\left(B_{R}^{f_{L}} \bar{f} \bar{\sigma}_{\mu} f+B_{R}^{f_{R}} f^{c} \sigma_{\mu} \bar{f}^{c}\right)+\text { h.c. }\right] .
$$

Here we have retained chirality conserving operators only and neglected chirality flipping ones, e.g. $\left(\mu^{c} \tau\right)\left(f f^{c}\right)$, because the latter are expected to suffer from a double chiral suppression and so would give subleading contributions to our LFV processes (in particular this holds in the MSSM, even for large $\tan \beta$ ). We emphasize that eq. (14) does not yet include either the contributions induced by $Z$ and photon exchange, to be added in the next section, or the chirality flipping operators induced by Higgs exchange, which are relevant for large $\tan \beta$ and will be discussed in Section 3.2.

\footnotetext{
${ }^{2}$ Sometimes this feature has been overlooked in the literature, by assuming the chirality conserving operators to be dominated by the zero-momentum component, i.e. $A^{Z}$ in our notation (see e.g. [13]).

${ }^{3}$ Another discussion on the phenomenological implications of $\mu-\tau$ LFV four-fermion operators appeared in $[14]$.
} 


\subsection{Effective lagrangians and branching ratios}

All the coefficients of the operators listed above $\left(A^{Z}, C^{Z}, D^{Z}, C^{\gamma}, D^{\gamma}, B^{f}\right)$ have mass dimension -2 , and encode information on the underlying physics. Such coefficients are the building blocks for the amplitudes of our LFV processes, as schematically shown in Fig. 1. Now we present for each LFV decay the relevant lagrangian and the corresponding branching ratio.
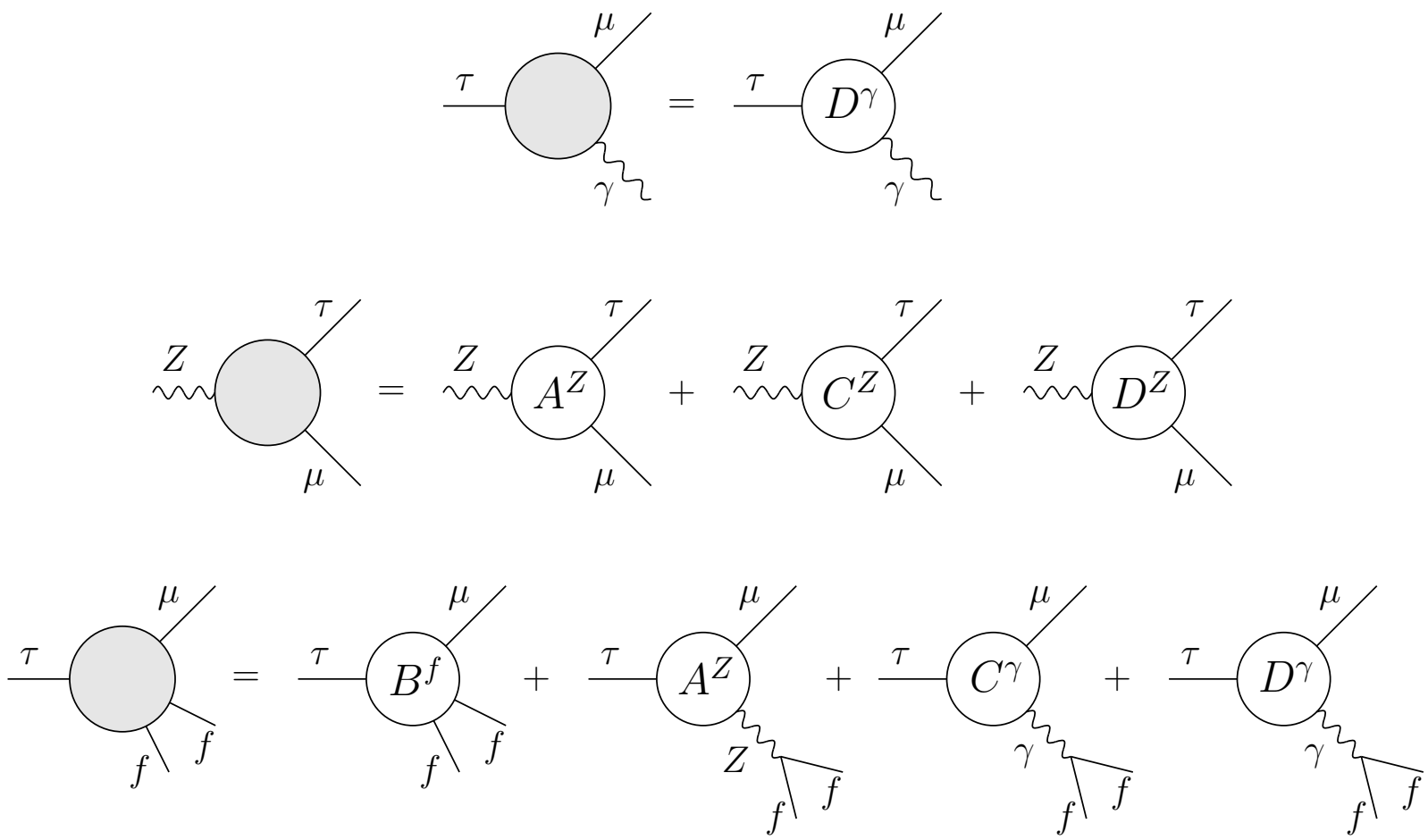

Figure 1: The different contributions to $\tau \rightarrow \mu \gamma, Z \rightarrow \mu \tau$ and $\tau \rightarrow \mu f f$ decays.

$\underline{Z \rightarrow \mu \tau}$

The effective lagrangian for the $Z \rightarrow \mu \tau$ decay is easily obtained from eqs. (8-10):

$$
\mathcal{L}_{Z \mu \tau}^{\mathrm{eff}}=g_{Z}\left[m_{Z}^{2}\left(F_{L}^{Z} \bar{\mu} \bar{\sigma}^{\mu} \tau+F_{R}^{Z} \mu^{c} \sigma^{\mu} \bar{\tau}^{c}\right) Z_{\mu}-2 m_{\tau}\left(D_{L}^{Z} \bar{\mu} \bar{\sigma}^{\mu \nu} \bar{\tau}^{c}+D_{R}^{Z} \mu^{c} \sigma^{\mu \nu} \tau\right) i \partial_{\nu} Z_{\mu}\right]+\text { h.c. }
$$

where

$$
F_{L(R)}^{Z}=A_{L(R)}^{Z}-C_{L(R)}^{Z}
$$

The branching ratio reads as:

$$
B R\left(Z \rightarrow \mu^{+} \tau^{-}\right)=c m_{Z}^{4}\left[\left|F_{L}^{Z}\right|^{2}+\left|F_{R}^{Z}\right|^{2}+\frac{1}{2}\left|\frac{m_{\tau}}{m_{Z}} D_{L}^{Z}\right|^{2}+\frac{1}{2}\left|\frac{m_{\tau}}{m_{Z}} D_{R}^{Z}\right|^{2}\right] B R\left(Z \rightarrow \ell^{+} \ell^{-}\right)
$$


where $c \equiv\left(1 / 4-s_{W}^{2}+2 s_{W}^{4}\right)^{-1} \simeq 7.9, B R\left(Z \rightarrow \ell^{+} \ell^{-}\right) \simeq 3.4 \%$ and lepton masses have been neglected in the kinematics ${ }^{4}$.

$\tau \rightarrow \mu \gamma$

In this case the expression of the effective lagrangian is already in eq. (13). The related branching ratio is:

$$
B R\left(\tau^{-} \rightarrow \mu^{-} \gamma\right)=\frac{48 \pi^{3} \alpha}{G_{F}^{2}}\left[\left|D_{L}^{\gamma}\right|^{2}+\left|D_{R}^{\gamma}\right|^{2}\right] B R\left(\tau^{-} \rightarrow \mu^{-} \bar{\nu}_{\mu} \nu_{\tau}\right)
$$

where $\alpha=e^{2} /(4 \pi)$ is the fine-structure constant, $G_{F}$ is the Fermi constant and $B R\left(\tau^{-} \rightarrow\right.$ $\left.\mu^{-} \bar{\nu}_{\mu} \nu_{\tau}\right) \simeq 17 \%$.

$\tau \rightarrow \mu \ell^{+} \ell^{-}, \tau \rightarrow \mu \rho, \tau \rightarrow \mu P\left(P=\pi, \eta, \eta^{\prime}\right)$

The four-fermion effective lagrangian relevant for these decays is obtained by combining the contributions in eq. (14) with those induced by $Z$ and photon exchange (see also Fig. 1). Other contributions, induced by Higgs boson exchange, will be discussed in Section 3.2. We have:

$$
\begin{aligned}
\mathcal{L}_{\tau \mu f f}^{\mathrm{eff}} & =\sum_{f}\left[\left(\bar{\mu} \bar{\sigma}^{\mu} \tau\right)\left(F_{L}^{f_{L}} \bar{f} \bar{\sigma}_{\mu} f+F_{L}^{f_{R}} f^{c} \sigma_{\mu} \bar{f}^{c}\right)+\left(\mu^{c} \sigma^{\mu} \bar{\tau}^{c}\right)\left(F_{R}^{f_{L}} \bar{f} \bar{\sigma}_{\mu} f+F_{R}^{f_{R}} f^{c} \sigma_{\mu} \bar{f}^{c}\right)\right] \\
& -2 e^{2}\left(D_{L}^{\gamma} \bar{\mu} \bar{\sigma}^{\mu \nu} \bar{\tau}^{c}+D_{R}^{\gamma} \mu^{c} \sigma^{\mu \nu} \tau\right) \frac{m_{\tau} i \partial_{\nu}}{\square} \sum_{f} Q_{f}\left(\bar{f} \bar{\sigma}_{\mu} f+f^{c} \sigma_{\mu} \bar{f}^{c}\right)+\text { h.c. }
\end{aligned}
$$

where

$$
\begin{aligned}
& F_{L(R)}^{f_{L}}=B_{L(R)}^{f_{L}}+\left(T_{f_{L}}^{3}-Q_{f} s_{W}^{2}\right) g_{Z}^{2} A_{L(R)}^{Z}+Q_{f} e^{2} C_{L(R)}^{\gamma} \\
& F_{L(R)}^{f_{R}}=B_{L(R)}^{f_{R}}-Q_{f} s_{W}^{2} g_{Z}^{2} A_{L(R)}^{Z}+Q_{f} e^{2} C_{L(R)}^{\gamma} .
\end{aligned}
$$

For the sake of brevity, we will often refer to the operators in the first line of eq. (19) as 'monopole' ones, to be contrasted with the 'dipole' ones in the second line.

The effective lagrangian (19) can be applied either to leptonic transitions, such as the decays $\tau^{-} \rightarrow \mu^{-} e^{+} e^{-}$and $\tau^{-} \rightarrow \mu^{-} \mu^{+} \mu^{-}$, or to semileptonic transitions involving hadrons, after taking the appropriate matrix elements of quark bilinears. In the processes involving a neutral pseudoscalar meson $P\left(P=\pi, \eta, \eta^{\prime}\right)$ the relevant matrix elements are $\left\langle 0\left|J_{5}^{\mu a}(0)\right| P(p)\right\rangle=i f_{P}^{a} p^{\mu}$ $(a=0,3,8)$, where $f_{P}^{a}$ are the decay constants and $J_{5}^{\mu a}$ are the axial-vector currents ${ }^{5}$. Among the neutral vector mesons, we have selected the $\rho$ meson and so the relevant matrix element is $\left\langle 0\left|J^{\mu 3}(0)\right| \rho\right\rangle=\kappa_{\rho} m_{\rho}^{2} \epsilon^{\mu}$, where $J^{\mu 3}$ is the third component of the vector current, $\kappa_{\rho} \simeq 0.2$ and $m_{\rho}, \epsilon^{\mu}$ are the $\rho$ mass and polarization vector, respectively. Our results can be easily extended to the other vector mesons, $\phi$ and $\omega$.

\footnotetext{
${ }^{4}$ Notice that the factors $m_{\tau}$ in eq. (17) come from the $D^{Z}$-operators, which may contain a (compensating) large $\tan \beta$ factor, as mentioned above.

${ }^{5}$ In terms of four-component spinors $\psi_{q}^{T}=\left(q \bar{q}^{c}\right)(q=u, d, s)$, collected in a triplet $\Psi^{T}=\left(\psi_{u}^{T} \psi_{d}^{T} \psi_{s}^{T}\right)$, the vector and axial-vector currents read as $J^{\mu a}=\bar{\Psi} \gamma^{\mu} \frac{\lambda^{a}}{2} \Psi$ and $J_{5}^{\mu a}=\bar{\Psi} \gamma^{\mu} \gamma^{5} \frac{\lambda^{a}}{2} \Psi(a=0,1, \ldots, 8)$, respectively. The neutral currents contain the matrices $\lambda^{0}=\sqrt{\frac{2}{3}} \operatorname{diag}(1,1,1), \lambda^{3}=\operatorname{diag}(1,-1,0), \lambda^{8}=\frac{1}{\sqrt{3}} \operatorname{diag}(1,1,-2)$.
} 
- $\tau \rightarrow \mu e e, \tau \rightarrow \mu \mu \mu$

The branching ratios are directly computed from eq. (19):

$$
\begin{aligned}
B R\left(\tau^{-} \rightarrow \mu^{-} e^{+} e^{-}\right) & =\frac{1}{8 G_{F}^{2}}\left[\left|F_{L}^{e_{L}}\right|^{2}+\left|F_{L}^{e_{R}}\right|^{2}+\left|F_{R}^{e_{L}}\right|^{2}+\left|F_{R}^{e_{R}}\right|^{2}\right. \\
& +4 e^{2} \operatorname{Re}\left(D_{L}^{\gamma}\left(\bar{F}_{L}^{e_{L}}+\bar{F}_{L}^{e_{R}}\right)+D_{R}^{\gamma}\left(\bar{F}_{R}^{e_{L}}+\bar{F}_{R}^{e_{R}}\right)\right) \\
& \left.+8 e^{4}\left(\left|D_{L}^{\gamma}\right|^{2}+\left|D_{R}^{\gamma}\right|^{2}\right)\left(\log \frac{m_{\tau}^{2}}{m_{e}^{2}}-3\right)\right] B R\left(\tau^{-} \rightarrow \mu^{-} \bar{\nu}_{\mu} \nu_{\tau}\right), \\
B R\left(\tau^{-} \rightarrow \mu^{-} \mu^{+} \mu^{-}\right)= & \frac{1}{8 G_{F}^{2}}\left[2\left|F_{L}^{\mu_{L}}\right|^{2}+\left|F_{L}^{\mu_{R}}\right|^{2}+\left|F_{R}^{\mu_{L}}\right|^{2}+2\left|F_{R}^{\mu_{R}}\right|^{2}\right. \\
& +4 e^{2} \operatorname{Re}\left(D_{L}^{\gamma}\left(2 \bar{F}_{L}^{\mu_{L}}+\bar{F}_{L}^{\mu_{R}}\right)+D_{R}^{\gamma}\left(\bar{F}_{R}^{\mu_{L}}+2 \bar{F}_{R}^{\mu_{R}}\right)\right) \\
& \left.+8 e^{4}\left(\left|D_{L}^{\gamma}\right|^{2}+\left|D_{R}^{\gamma}\right|^{2}\right)\left(\log \frac{m_{\tau}^{2}}{m_{\mu}^{2}}-\frac{11}{4}\right)\right] B R\left(\tau^{-} \rightarrow \mu^{-} \bar{\nu}_{\mu} \nu_{\tau}\right) .
\end{aligned}
$$

The electron mass $m_{e}$ and the muon mass $m_{\mu}$ regularize an infrared singularity in phase space in $\tau \rightarrow \mu e e$ and $\tau \rightarrow \mu \mu \mu$, respectively.

- $\tau \rightarrow \mu \rho$

The effective lagrangian for $\tau \rightarrow \mu \rho$ is also obtained from eq. (19):

$$
\mathcal{L}_{\tau \mu \rho}^{\mathrm{eff}}=\kappa_{\rho}\left[m_{\rho}^{2}\left(F_{L}^{\rho} \bar{\mu} \bar{\sigma}^{\mu} \tau+F_{R}^{\rho} \mu^{c} \sigma^{\mu} \bar{\tau}^{c}\right) \rho_{\mu}^{0}+2 e^{2} m_{\tau}\left(D_{L}^{\gamma} \bar{\mu} \bar{\sigma}^{\mu \nu} \bar{\tau}^{c}+D_{R}^{\gamma} \mu^{c} \sigma^{\mu \nu} \tau\right) i \partial_{\nu} \rho_{\mu}^{0}\right]+\text { h.c. }
$$

where

$$
\begin{aligned}
F_{L(R)}^{\rho} & =\frac{1}{2}\left(F_{L(R)}^{u_{L}}-F_{L(R)}^{d_{L}}\right)+\frac{1}{2}\left(F_{L(R)}^{u_{R}}-F_{L(R)}^{d_{R}}\right) \\
& =\frac{1}{2}\left[\left(B_{L(R)}^{u_{L}}-B_{L(R)}^{d_{L}}\right)+\left(B_{L(R)}^{u_{R}}-B_{L(R)}^{d_{R}}\right)+\left(1-2 s_{W}^{2}\right) g_{Z}^{2} A_{L(R)}^{Z}+2 e^{2} C_{L(R)}^{\gamma}\right] .
\end{aligned}
$$

The related branching ratio is:

$$
\begin{aligned}
B R\left(\tau^{-} \rightarrow \mu^{-} \rho^{0}\right) & =\frac{1}{4 G_{F}^{2} c_{c}^{2}}\left[\left|F_{L}^{\rho}\right|^{2}+\left|F_{R}^{\rho}\right|^{2}-\frac{6 e^{2}}{1+2 x_{\rho}} \operatorname{Re}\left(D_{L}^{\gamma} \bar{F}_{L}^{\rho}+D_{R}^{\gamma} \bar{F}_{R}^{\rho}\right)\right. \\
& \left.+\frac{e^{4}\left(2+x_{\rho}\right)}{x_{\rho}\left(1+2 x_{\rho}\right)}\left(\left|D_{L}^{\gamma}\right|^{2}+\left|D_{R}^{\gamma}\right|^{2}\right)\right] B R\left(\tau^{-} \rightarrow \nu_{\tau} \rho^{-}\right)
\end{aligned}
$$

where $c_{c}^{2} \equiv \cos ^{2} \theta_{c} \simeq 0.95, x_{\rho} \equiv m_{\rho}^{2} / m_{\tau}^{2} \simeq 0.19$ and $B R\left(\tau^{-} \rightarrow \nu_{\tau} \rho^{-}\right) \simeq 25 \%$. 
- $\tau \rightarrow \mu \pi$

From eq. (19) we also derive the effective lagrangian for $\tau \rightarrow \mu \pi$. It does not receive contributions from photon exchange, and reads as:

$$
\mathcal{L}_{\tau \mu \pi}^{\mathrm{eff}}=f_{\pi}\left(F_{L}^{\pi} \bar{\mu} \bar{\sigma}^{\mu} \tau+F_{R}^{\pi} \mu^{c} \sigma^{\mu} \bar{\tau}^{c}\right) \partial_{\mu} \pi^{0}+\text { h.c. }
$$

where $f_{\pi} \equiv f_{\pi}^{3} \simeq 92 \mathrm{MeV}$ (we neglect $\left.f_{\pi}^{0}, f_{\pi}^{8}\right)$ and

$$
\begin{aligned}
F_{L(R)}^{\pi} & =\frac{1}{2}\left(F_{L(R)}^{u_{L}}-F_{L(R)}^{d_{L}}\right)-\frac{1}{2}\left(F_{L(R)}^{u_{R}}-F_{L(R)}^{d_{R}}\right) \\
& =\frac{1}{2}\left[\left(B_{L(R)}^{u_{L}}-B_{L(R)}^{d_{L}}\right)-\left(B_{L(R)}^{u_{R}}-B_{L(R)}^{d_{R}}\right)+g_{Z}^{2} A_{L(R)}^{Z}\right] .
\end{aligned}
$$

The branching ratio is:

$$
B R\left(\tau^{-} \rightarrow \mu^{-} \pi^{0}\right)=\frac{1}{4 G_{F}^{2} c_{c}^{2}}\left[\left|F_{L}^{\pi}\right|^{2}+\left|F_{R}^{\pi}\right|^{2}\right] B R\left(\tau^{-} \rightarrow \nu_{\tau} \pi^{-}\right)
$$

where $B R\left(\tau^{-} \rightarrow \nu_{\tau} \pi^{-}\right) \simeq 11 \%$.

- $\tau \rightarrow \mu \eta, \tau \rightarrow \mu \eta^{\prime}$

Each of the two mesons $\eta$ and $\eta^{\prime}$ has both octet and singlet $S U(3)_{\text {flav }}$ components. The relevant decay constants are $f_{P}^{a}$, where $P=\eta, \eta^{\prime}$ and $a=8,0$ (we neglect $f_{P}^{3}$ ) $[15,16]$. For the ratios $f_{P}^{a} / f_{\pi}$ we take the following numerical values [16]: $f_{\eta}^{8} \sim 1.2 f_{\pi}, f_{\eta}^{0} \sim$ $0.2 f_{\pi}, f_{\eta^{\prime}}^{8} \sim-0.45 f_{\pi}, f_{\eta^{\prime}}^{0} \sim 1.15 f_{\pi}$.

The effective lagrangian for $\tau \rightarrow \mu \eta$, which does not receive contributions from photon exchange, is derived from eq. (19). It reads as:

$$
\mathcal{L}_{\tau \mu \eta}^{\mathrm{eff}}=\left[\left(f_{\eta}^{8} F_{L}^{\eta, 8}+f_{\eta}^{0} F_{L}^{\eta, 0}\right) \bar{\mu} \bar{\sigma}^{\mu} \tau+\left(f_{\eta}^{8} F_{R}^{\eta, 8}+f_{\eta}^{0} F_{R}^{\eta, 0}\right) \mu^{c} \sigma^{\mu} \bar{\tau}^{c}\right] \partial_{\mu} \eta+\text { h.c. }
$$

where

$$
\begin{aligned}
F_{L(R)}^{\eta, 8} & =\frac{1}{2 \sqrt{3}}\left(F_{L(R)}^{u_{L}}+F_{L(R)}^{d_{L}}-2 F_{L(R)}^{s_{L}}\right)-\frac{1}{2 \sqrt{3}}\left(F_{L(R)}^{u_{R}}+F_{L(R)}^{d_{R}}-2 F_{L(R)}^{s_{R}}\right) \\
& =\frac{1}{2 \sqrt{3}}\left[\left(B_{L(R)}^{u_{L}}+B_{L(R)}^{d_{L}}-2 B_{L(R)}^{s_{L}}\right)-\left(B_{L(R)}^{u_{R}}+B_{L(R)}^{d_{R}}-2 B_{L(R)}^{s_{R}}\right)+g_{Z}^{2} A_{L(R)}^{Z}\right] \\
F_{L(R)}^{\eta, 0} & =\frac{1}{\sqrt{6}}\left(F_{L(R)}^{u_{L}}+F_{L(R)}^{d_{L}}+F_{L(R)}^{s_{L}}\right)-\frac{1}{\sqrt{6}}\left(F_{L(R)}^{u_{R}}+F_{L(R)}^{d_{R}}+F_{L(R)}^{s_{R}}\right) \\
& =\frac{1}{\sqrt{6}}\left[\left(B_{L(R)}^{u_{L}}+B_{L(R)}^{d_{L}}+B_{L(R)}^{s_{L}}\right)-\left(B_{L(R)}^{u_{R}}+B_{L(R)}^{d_{R}}+B_{L(R)}^{s_{R}}\right)-\frac{1}{2} g_{Z}^{2} A_{L(R)}^{Z}\right] .
\end{aligned}
$$

The branching ratio can be expressed as:

$$
B R\left(\tau^{-} \rightarrow \mu^{-} \eta\right)=\frac{1}{4 G_{F}^{2} c_{c}^{2}}\left[\left|\frac{f_{\eta}^{8}}{f_{\pi}} F_{L}^{\eta, 8}+\frac{f_{\eta}^{0}}{f_{\pi}} F_{L}^{\eta, 0}\right|^{2}+(L \rightarrow R)\right]\left(1-x_{\eta}\right)^{2} B R\left(\tau^{-} \rightarrow \nu_{\tau} \pi^{-}\right)
$$


where $x_{\eta} \equiv m_{\eta}^{2} / m_{\tau}^{2} \simeq 9.5 \times 10^{-2}$ and the ratio $m_{\pi}^{2} / m_{\tau}^{2}$ has been neglected. For the decay $\tau \rightarrow \mu \eta^{\prime}$, one has to make the replacement $\eta \rightarrow \eta^{\prime}$ in eqs. (30), (31), (32) and (33). We anticipate that the equalities $F_{L(R)}^{\eta, 8}=F_{L(R)}^{\eta^{\prime}, 8}, F_{L(R)}^{\eta, 0}=F_{L(R)}^{\eta^{\prime}, 0}$, which follow from eq. (19), will not hold in the presence of sizeable Higgs-mediated contributions [see Section 3.2].

\subsection{Correlations}

In the previous subsection we have seen that, on the one hand, each operator can contribute to different processes, hence correlations exist. On the other hand, the correlation pattern is not trivial because each process can get contributions from different operators, so making general predictions is not straightforward. Nevertheless, it is interesting to see what happens when several processes are dominated by the same operator. Such a situation may or may not be realized in a specific underlying model or in a specific portion of the model parameter space.

$D^{\gamma}$-dominance. If photon dipole contributions are the dominant ones in the decays $\tau \rightarrow$ $\mu e e, \tau \rightarrow \mu \mu \mu$ and $\tau \rightarrow \mu \rho$, then such processes can directly be compared to $\tau \rightarrow \mu \gamma$ :

$$
\begin{aligned}
\frac{B R\left(\tau^{-} \rightarrow \mu^{-} e^{+} e^{-}\right)}{B R\left(\tau^{-} \rightarrow \mu^{-} \gamma\right)} & \simeq \frac{\alpha}{3 \pi}\left(\log \frac{m_{\tau}^{2}}{m_{e}^{2}}-3\right) \simeq 10^{-2} \\
\frac{B R\left(\tau^{-} \rightarrow \mu^{-} \mu^{+} \mu^{-}\right)}{B R\left(\tau^{-} \rightarrow \mu^{-} \gamma\right)} & \simeq \frac{\alpha}{3 \pi}\left(\log \frac{m_{\tau}^{2}}{m_{\mu}^{2}}-\frac{11}{4}\right) \simeq 2.2 \times 10^{-3} \\
\frac{B R\left(\tau^{-} \rightarrow \mu^{-} \rho^{0}\right)}{B R\left(\tau^{-} \rightarrow \mu^{-} \gamma\right)} & \simeq \frac{\alpha}{12 \pi c_{c}^{2}} \frac{\left(2+x_{\rho}\right)}{x_{\rho}\left(1+2 x_{\rho}\right)} \frac{B R\left(\tau^{-} \rightarrow \nu_{\tau} \rho^{-}\right)}{B R\left(\tau^{-} \rightarrow \mu^{-} \bar{\nu}_{\mu} \nu_{\tau}\right)} \simeq 2.5 \times 10^{-3}
\end{aligned}
$$

In particular, the present bound (1) on $\tau \rightarrow \mu \gamma$ would imply the following bounds:

$$
\begin{aligned}
B R\left(\tau^{-} \rightarrow \mu^{-} e^{+} e^{-}\right) & \lesssim 3 \times 10^{-9}, \\
B R\left(\tau^{-} \rightarrow \mu^{-} \mu^{+} \mu^{-}\right) & \lesssim 7 \times 10^{-10}, \\
B R\left(\tau^{-} \rightarrow \mu^{-} \rho^{0}\right) & \lesssim 8 \times 10^{-10}
\end{aligned}
$$

$C^{\gamma}$-dominance. Suppose now that $D^{\gamma}$-contributions are suppressed and that monopole contributions to $\tau \rightarrow \mu e e, \tau \rightarrow \mu \mu \mu, \tau \rightarrow \mu \rho$ are dominated by $C^{\gamma}$-operators. Then:

$$
B R\left(\tau^{-} \rightarrow \mu^{-} \rho^{0}\right) \simeq B R\left(\tau^{-} \rightarrow \mu^{-} \mu^{+} \mu^{-}\right) \simeq 1.5 \times B R\left(\tau^{-} \rightarrow \mu^{-} e^{+} e^{-}\right) .
$$

$A^{Z}$-dominance. Suppose again that dipole contributions are suppressed and that monopole contributions to $\tau \rightarrow \mu e e, \tau \rightarrow \mu \mu \mu, \tau \rightarrow \mu \rho, \tau \rightarrow \mu \pi, \tau \rightarrow \mu \eta, \tau \rightarrow \mu \eta^{\prime}$ and $Z \rightarrow \mu \tau$ are dominated by $A^{Z}$-operators. Then:

$$
\begin{aligned}
B R\left(Z \rightarrow \mu^{+} \tau^{-}\right) & \simeq 3 \times B R\left(\tau^{-} \rightarrow \mu^{-} e^{+} e^{-}\right) \\
B R\left(\tau^{-} \rightarrow \mu^{-} \rho^{0}\right) & \simeq 1.8 \times B R\left(\tau^{-} \rightarrow \mu^{-} e^{+} e^{-}\right) \\
B R\left(\tau^{-} \rightarrow \mu^{-} \pi^{0}\right) & \simeq 2.7 \times B R\left(\tau^{-} \rightarrow \mu^{-} e^{+} e^{-}\right)
\end{aligned}
$$




$$
\begin{aligned}
B R\left(\tau^{-} \rightarrow \mu^{-} \eta\right) & \simeq 0.8 \times B R\left(\tau^{-} \rightarrow \mu^{-} e^{+} e^{-}\right) \\
B R\left(\tau^{-} \rightarrow \mu^{-} \eta^{\prime}\right) & \simeq 0.7 \times B R\left(\tau^{-} \rightarrow \mu^{-} e^{+} e^{-}\right) \\
B R\left(\tau^{-} \rightarrow \mu^{-} \mu^{+} \mu^{-}\right) & \simeq\left(\begin{array}{l}
1.6 \\
1.4
\end{array}\right) \times B R\left(\tau^{-} \rightarrow \mu^{-} e^{+} e^{-}\right) .
\end{aligned}
$$

The result for $\tau \rightarrow \mu \mu \mu$ depends on the relative amount of $A_{L}^{Z}$ and $A_{R}^{Z}$ contributions: the upper (lower) estimate refers to the case of pure $A_{L}^{Z}\left(A_{R}^{Z}\right)$ dominance, with $A_{R}^{Z}=0$ $\left(A_{L}^{Z}=0\right)$.

$B^{f}$-dominance. Finally, consider the case in which all contributions are suppressed except for those $B^{f}$-induced. Then correlations are in general weakened or lost, since different $B^{f}$ coefficients appear in different processes. In some specific case, however, simple correlations may emerge. For example, consider the limit of flavour universality in the down-quark sector, i.e. $B_{L(R)}^{d_{L}}=B_{L(R)}^{s_{L}}$ and $B_{L(R)}^{d_{R}}=B_{L(R)}^{s_{R}}$ (which holds in the MSSM with down-squark mass universality). Then we obtain $B R(\tau \rightarrow \mu \pi) \sim 3 \times B R(\tau \rightarrow \mu \eta)$ by neglecting $f_{\eta}^{0}$ in eq. (33). Another example of correlation is that between $\tau \rightarrow \mu \rho$ and $\tau \rightarrow \mu \pi$ in the absence of LFV in the left sector $\left(B_{L}^{u_{L(R)}}=B_{L}^{d_{L(R)}}=0\right)$. In this case, in the $S U(2)_{W}$-symmetric limit $\left(B_{R}^{u_{L}}=B_{R}^{d_{L}}\right)$, both decays only depend on the combination $B_{R}^{u_{R}}-B_{R}^{d_{R}}$, hence $B R(\tau \rightarrow \mu \rho) \sim 2.3 \times B R(\tau \rightarrow \mu \pi)$.

\section{The MSSM framework}

In the above sections we have given a model-independent description of effective operators that contribute to our selected set of LFV processes. Here our purpose is to compute the coefficients of such operators in the framework of the MSSM. The source of LFV in the MSSM is the potential misalignment between the lepton and slepton mass matrices. More precisely, in the superfield basis in which the charged lepton mass matrix is diagonal, the sources of LFV are the off-diagonal entries of the soft-breaking matrices $\tilde{\mathcal{M}}_{L}^{2}, \tilde{\mathcal{M}}_{R}^{2}$ and $\tilde{\mathcal{A}}$. If we restrict our attention to the second/third generation, the latter matrices have the form (see also Appendix A.1):

$$
\tilde{\mathcal{M}}_{L}^{2}=\left(\begin{array}{cc}
\tilde{m}_{L \mu \mu}^{2} & \tilde{m}_{L \mu \tau}^{2} \\
\tilde{m}_{L \mu \tau}^{2 *} & \tilde{m}_{L \tau \tau}^{2}
\end{array}\right), \quad \tilde{\mathcal{M}}_{R}^{2}=\left(\begin{array}{cc}
\tilde{m}_{R \mu \mu}^{2} & \tilde{m}_{R \mu \tau}^{2} \\
\tilde{m}_{R \mu \tau}^{2 *} & \tilde{m}_{R \tau \tau}^{2}
\end{array}\right), \quad \tilde{\mathcal{A}}=\left(\begin{array}{cc}
h_{\mu} A_{\mu} & h_{\tau} A_{\mu \tau}^{R} \\
h_{\tau} A_{\mu \tau}^{L} & h_{\tau} A_{\tau}
\end{array}\right) .
$$

Notice that we have parametrized the entries of $\tilde{\mathcal{A}}$ by extracting a suitable Yukawa coupling (either $h_{\mu}$ or $\left.h_{\tau}\right)$. We will use the symbols $(\mathrm{LFV})_{L}$ and $(\mathrm{LFV})_{R}$ to characterize LFV in the left and right sectors, respectively:

$$
\begin{array}{llll}
(\mathrm{LFV})_{L}: & \tilde{m}_{L \mu \tau}^{2} \neq 0 \quad \text { and } / \text { or } & A_{\mu \tau}^{L} \neq 0, \\
(\mathrm{LFV})_{R}: & \tilde{m}_{R \mu \tau}^{2} \neq 0 \quad \text { and/or } & A_{\mu \tau}^{R} \neq 0 .
\end{array}
$$

In the case of pure $(\mathrm{LFV})_{L}\left[(\mathrm{LFV})_{R}\right]$ the parameter $A_{\mu \tau}^{R}\left(A_{\mu \tau}^{L}\right)$ is expected to be suppressed by $h_{\mu} / h_{\tau}$ with respect to $A_{\mu \tau}^{L}\left(A_{\mu \tau}^{R}\right)$. 
In Section 3.1 we describe some general features of the MSSM contributions to the operator coefficients listed in Section 2.1. We also present the Higgs- $\mu-\tau$ effective operators and discuss their impact on $\tau \rightarrow 3 \mu, \tau \rightarrow \mu \pi, \tau \rightarrow \mu \eta, \tau \rightarrow \mu \eta^{\prime}$ (Section 3.2). Finally, we discuss the effect of LFV on the muon anomalous magnetic moment (Section 3.3). The explicit results of the calculations for the operator coefficients are presented in Appendices A.2-A.10.

\subsection{General aspects of operator contributions}

The leading contributions to the coefficients $A^{Z}, C^{Z}, C^{\gamma}, D^{Z}, D^{\gamma}, B^{f}$ arise from one-loop diagrams that involve the exchange of gauginos, Higgsinos and sleptons (and also $\tilde{f}$ sfermions in the case of $B^{f}$ ). These diagrams are shown schematically in Fig. 2 and in more detail in Appendices A.3-A.8, where the explicit analytical results for the coefficients are also presented. The relevant parameters involved are those in eq. (47) (or equivalent ones, see below), the $S U(2)_{W} \times U(1)_{Y}$ gaugino masses $\left(M_{2}, M_{1}\right)$, the $\mu$ parameter $^{6}$, other sfermion masses and $\tan \beta$. We neglect contributions to the LFV operator coefficients coming from other sources ${ }^{7}$.

In the diagrams in Fig. 2, the flavour transition occurs along the slepton line. The generic behaviour of the related operator coefficients at the linear order in the LFV parameters $\tilde{m}_{\mu \tau}^{2}, A_{\mu \tau}$ (the so-called mass-insertion approximation [18]) goes as follows:

$$
\begin{aligned}
A^{Z}, C^{\gamma}, C^{Z}, B^{f} / g_{w}^{2} & \sim \frac{g_{w}^{2}}{16 \pi^{2}} \cdot \frac{1}{M_{S}^{2}} \cdot\left(\frac{\tilde{m}_{\mu \tau}^{2}}{M_{S}^{2}}\right) \\
D^{\gamma}, D^{Z} & \sim \frac{g_{w}^{2}}{16 \pi^{2}} \cdot \frac{1}{M_{S}^{2}} \cdot\left(\frac{\tilde{m}_{\mu \tau}^{2}}{M_{S}^{2}} \text { or } \frac{\tilde{m}_{\mu \tau}^{2}}{M_{S}^{2}} \tan \beta \text { or } \frac{A_{\mu \tau}}{M_{S}}\right)
\end{aligned}
$$

where the constant $g_{w}$ can be either $g$ or $g^{\prime}$ and $M_{S}$ is some effective sparticle mass. These relations show that generically all the coefficients have the same order of magnitude (except for a possible $\tan \beta$-enhancement in the dipole coefficients), are suppressed by $M_{S}^{2}$ and are proportional to the relative amount of LFV. Nevertheless, detailed computations are, of course, necessary to single out the specific dependence of each coefficient on the MSSM parameters. Upon addressing such a computation one is concerned, in particular, with the treatment of LFV. Since LFV in the second/third generation may not be a small effect, we choose to go beyond the mass-insertion approximation. Specifically, we diagonalize the mass matrices $\tilde{\mathcal{M}}_{L}^{2}, \tilde{\mathcal{M}}_{R}^{2}$ and work with their mass eigenstates, i.e. our results are expressed in terms of the corresponding eigenvalues $\tilde{m}_{L_{\alpha}}^{2}, \tilde{m}_{R_{\alpha}}^{2}(\alpha=2,3)$ and mixing angles $\theta_{L}, \theta_{R}$. In particular, large $(\mathrm{LFV})_{L}$ in $\tilde{\mathcal{M}}_{L}^{2}$ means $\tilde{m}_{L \mu \tau}^{2} \sim \tilde{m}_{L \mu \mu}^{2} \sim \tilde{m}_{L \tau \tau}^{2}$, which in turn implies $\theta_{L} \sim \mathcal{O}(1)$ and $\left(\tilde{m}_{L_{2}}^{2}-\tilde{m}_{L_{3}}^{2}\right) /\left(\tilde{m}_{L_{2}}^{2}+\tilde{m}_{L_{3}}^{2}\right) \sim$ $\mathcal{O}(1)$ (with $\tilde{m}_{L_{2}}^{2}, \tilde{m}_{L_{3}}^{2}$ of the same order or hierarchical). Analogous relations hold for the case of large $(\mathrm{LFV})_{R}$. Another aspect we have to deal with is the inclusion of electroweak breaking effects. We choose to treat such effects at lowest order, i.e. the only Higgs insertions we consider are those explicitly depicted in the diagrams in Fig. 2 (and also in Appendix), which

\footnotetext{
${ }^{6}$ We take a superpotential term of the form $W=\mu H_{1} H_{2}=\mu\left(H_{1}^{0} H_{2}^{0}-H_{1}^{-} H_{2}^{+}\right)$, so our sign convention for the $\mu$ parameter is opposite to the one commonly used (for this and other conventions see also Appendix A.1).

${ }^{7}$ Additional contributions arise, for instance, from the interactions of the MSSM fields with the goldstino supermultiplet [17]. Such effects are suppressed, unless the supersymmetry breaking scale $\sqrt{F}$ is close to the electroweak scale.
} 

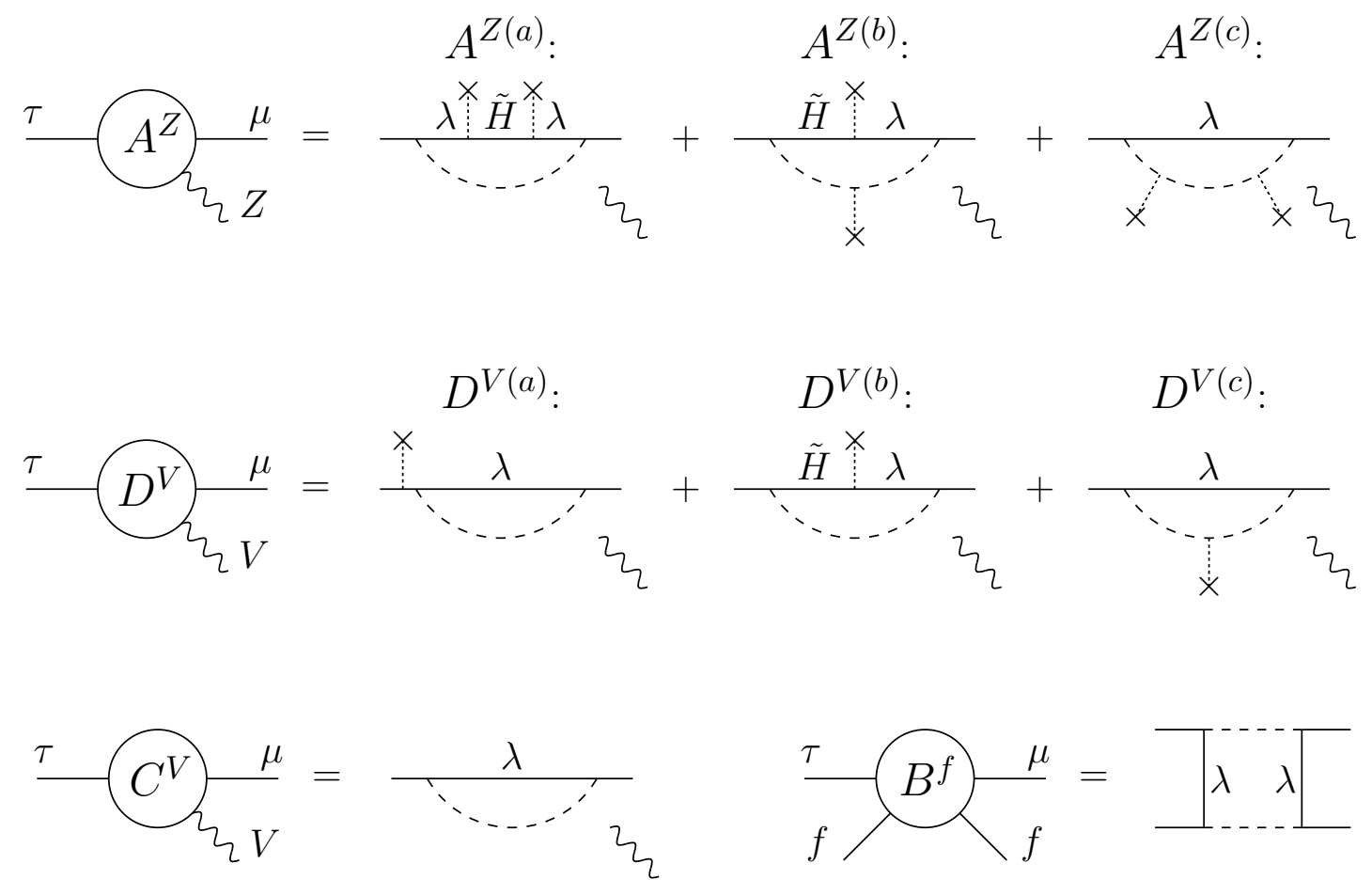

Figure 2: Topologies of the MSSM diagrams contributing to the coefficients $A^{Z}, D^{V}, C^{V}(V=\gamma, Z)$ and $B^{f}$. Internal solid lines denote gauginos $(\lambda)$ or higgsinos $(\tilde{H})$. Dashed lines denote sleptons (or other sfermions in the box diagrams). Dotted lines with a cross denote Higgs insertions.

also corresponds to take only the $d=6$ operators in the $S U(2)_{W} \times U(1)_{Y^{-}}$-unbroken phase. We believe that working in this approximation is more transparent and appealing from the theoretical point of view, although this may imply some loss of numerical accuracy when $M_{S}^{2}$ is very close to $m_{Z}^{2}$.

A few additional comments are in order about some operator coefficients. In particular, we recall that $A^{Z}$ is associated to operators like those in eq. (11), where the $Z_{\mu}$ field is contained in a covariant derivative acting on a Higgs field $\left(D_{\mu} H\right)$. Thus the operator coefficient can also be extracted from the part of the operator containing an ordinary derivative $\left(\partial_{\mu} H\right)$, that is from diagrams with momentum dependent Higgs lines and no $Z$ lines. This method, which we have used, has the advantage that one need not compute LFV diagrams corresponding to wavefunction renormalization of lepton fields, which are associated to operators like $\left(\bar{L}_{\mu} \bar{\sigma}^{\nu} D_{\nu} L_{\tau}\right)$ with or without Higgs fields attached. Also notice that $A^{Z}$ receives contributions of three different types:

$$
A_{L, R}^{Z}=A_{L, R}^{Z(a)}+A_{L, R}^{Z(b)}+A_{L, R}^{Z(c)}
$$

The terms $A_{L, R}^{Z(b)}, A_{L, R}^{Z(c)}$ are parametrically suppressed by $h_{\tau}^{2} / g_{w}^{2}$ with respect to $A_{L, R}^{Z(a)}$, hence those contributions can only be relevant for large $\tan \beta$. Also the dipole coefficients $D^{V}(V=\gamma, Z)$ 
receive contributions of three different types:

$$
D_{L, R}^{V}=D_{L, R}^{V(a)}+D_{L, R}^{V(b)}+D_{L, R}^{V(c)}
$$

Notice that the diagrams contributing to $D_{L, R}^{V(a)}$ can only contain the Higgs field $H_{1}$, which comes from the $\tau$ equation of motion, whereas either $H_{1}$ or $H_{2}$ can appear in the diagrams contributing to $D_{L, R}^{V(b)}, D_{L, R}^{V(c)}$. As a consequence only the latter coefficients can receive a $\tan \beta$ enhancement. In the case of $D^{Z}$ it is sufficient to compute these $\tan \beta$-enhanced terms, as they are the only ones which can give contributions comparable to the monopole ones in the $Z \rightarrow \mu \tau$ decay [see eq. (17)]. Notice that $\tan \beta$-enhanced terms have a more dramatic effect in the case of $D^{\gamma}$ [19], since they generically make the dipole operators dominate over the monopole ones in decays such as $\tau \rightarrow \mu e e, \tau \rightarrow \mu \mu \mu$ and $\tau \rightarrow \mu \rho$.

\subsection{Higgs-muon-tau effective interactions}

At large $\tan \beta$, another class of LFV interactions is relevant, namely those between a $\mu-\tau$ pair and Higgs bosons. For our purposes it is sufficient to focus on the leading effects, which arise from these dimension-four operators:

$$
-h_{\tau} \Delta_{L}^{*} H_{2}^{0 *} \tau^{c} \mu-h_{\tau} \Delta_{R} H_{2}^{0 *} \mu^{c} \tau+\text { h.c. }
$$

The corresponding diagrams are sketched in Fig. 3 and shown in more detail in Appendix A.9,
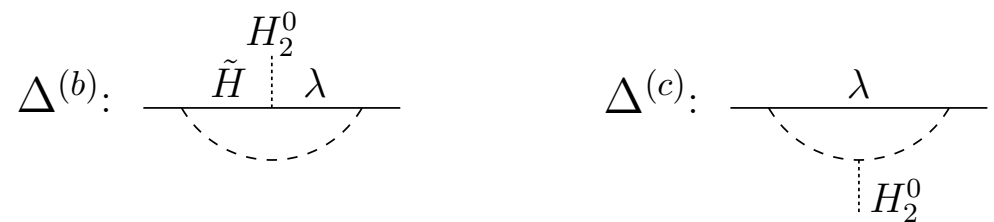

Figure 3: Topologies of the MSSM diagrams contributing to the $\Delta$ coefficients. Dashed lines denote sleptons.

where the explicit expressions of the dimensionless coefficients $\Delta_{L, R}=\Delta_{L, R}^{(b)}+\Delta_{L, R}^{(c)}$ are given ${ }^{8}$. In the mass-eigenstate basis for both leptons and Higgs bosons, the LFV couplings read as:

$$
\mathcal{L}_{\text {Higgs } \mu \tau}^{\text {eff }}=-\frac{h_{\tau}}{\sqrt{2} \mathrm{c}_{\beta}}\left(\Delta_{L}^{*} \tau^{c} \mu+\Delta_{R} \mu^{c} \tau\right)\left[\mathrm{c}_{\beta-\alpha} h-\mathrm{s}_{\beta-\alpha} H-\mathrm{i} A\right]+\text { h.c. },
$$

where $A$ is the physical CP-odd Higgs field, $\alpha$ is the mixing angle in the CP-even Higgs sector $\left[\sqrt{2} \operatorname{Re}\left(H_{1}^{0}-\left\langle H_{1}^{0}\right\rangle\right)=\mathrm{c}_{\alpha} H-\mathrm{s}_{\alpha} h, \sqrt{2} \operatorname{Re}\left(H_{2}^{0}-\left\langle H_{2}^{0}\right\rangle\right)=\mathrm{s}_{\alpha} H+\mathrm{c}_{\alpha} h\right]$, and a short-hand notation is used for $\mathrm{c}_{\xi}=\cos \xi, \mathrm{s}_{\xi}=\sin \xi(\xi=\alpha, \beta, \beta-\alpha)$. The effective couplings (55) directly contribute

\footnotetext{
${ }^{8}$ We should add that most of the properties discussed in this section do not rely on the specific MSSM origin of $\Delta_{L, R}$. The discussion can also apply, more generally, to two-Higgs-doublet models in which lepton masses are mainly generated through Yukawa couplings to $H_{1}$, with smaller contributions from $H_{2}$.
} 
to LFV decays of the neutral Higgs bosons, $A, H, h \rightarrow \mu \tau$ [11], and also induce Higgs mediated contributions to several LFV $\tau$ decays, such as $\tau \rightarrow 3 \mu[20,11], \tau \rightarrow \mu \eta[21], \tau \rightarrow \mu \eta^{\prime}$ and $\tau \rightarrow \mu \pi$. The $\Delta$-contributions to all such processes are displayed in Fig. 4 . Here we discuss each of them.
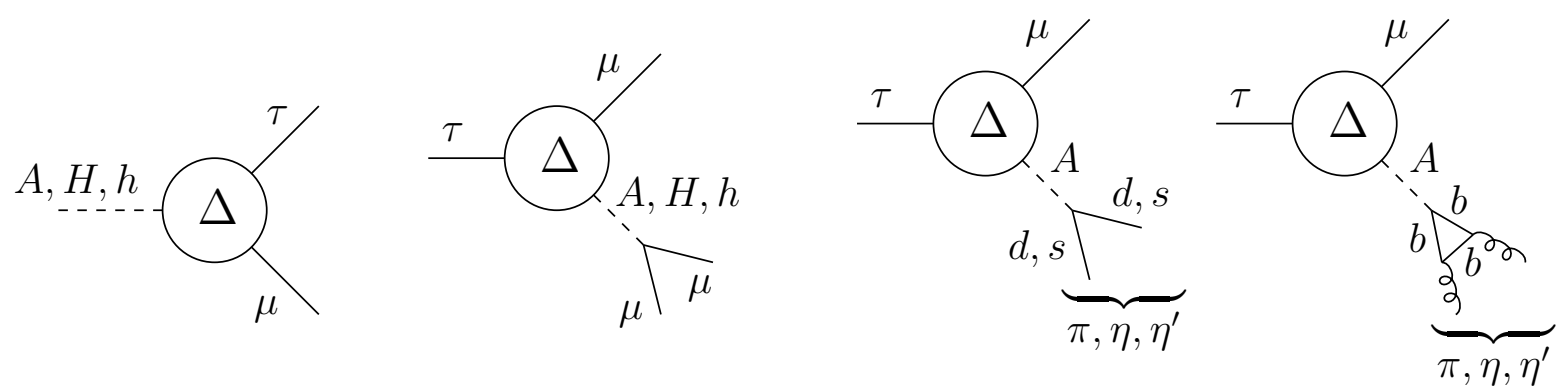

Figure 4: $\Delta$-contributions to the Higgs boson decays $A, H, h \rightarrow \mu \tau$ and to the decays $\tau \rightarrow 3 \mu, \tau \rightarrow \mu \pi$, $\tau \rightarrow \mu \eta, \tau \rightarrow \mu \eta^{\prime}$. In the last diagram, curly lines denote gluons.

- $\underline{\text { Higgs } \rightarrow \mu \tau}$

Concerning the Higgs boson decays, we have

$$
B R\left(A \rightarrow \mu^{+} \tau^{-}\right)=\tan ^{2} \beta\left(\left|\Delta_{L}\right|^{2}+\left|\Delta_{R}\right|^{2}\right) B R\left(A \rightarrow \tau^{+} \tau^{-}\right),
$$

where we have approximated $1 / \mathrm{c}_{\beta}^{2} \simeq \tan ^{2} \beta$ since non-negligible effects can only arise in the large $\tan \beta$ limit. If $A$ is replaced with $H$ [or $h]$ in eq. (56), the r.h.s. should also be multiplied by a factor $\left(\mathrm{c}_{\beta-\alpha} / \mathrm{s}_{\alpha}\right)^{2}\left[\right.$ or $\left.\left(\mathrm{s}_{\beta-\alpha} / \mathrm{c}_{\alpha}\right)^{2}\right]$. These LFV decays and the related phenomenology have been extensively investigated in [11] (for other studies in two-Higgs doublet models see e.g. [22]). We recall that $B R(A \rightarrow \mu \tau)$ can reach values of order $10^{-4}$. The same holds for the 'non-standard' CP-even Higgs boson (either $H$ or $h$, depending on $\left.m_{A}\right)$.

- $\tau \rightarrow \mu \mu \mu$

Consider now the implications of virtual Higgs exchange for $\tau \rightarrow \mu \mu \mu$. The effective lagrangian $\mathcal{L}_{\tau \mu \mu \mu}^{\text {eff }}$ [see eq. (19)] receives the following additional contribution from Higgs exchange:

$$
\delta \mathcal{L}_{\tau \mu \mu \mu}^{\mathrm{eff}}=-\sqrt{2} G_{F} m_{\mu} m_{\tau}\left(\mathcal{C}_{+} \bar{\mu} \bar{\mu}^{c}+\mathcal{C}_{-} \mu^{c} \mu\right)\left(\Delta_{R} \mu^{c} \tau+\Delta_{L}^{*} \mu \tau^{c}\right)+\text { h.c. },
$$

where the $\mathcal{C}_{ \pm}$coefficients are:

$$
\mathcal{C}_{ \pm}=\frac{1}{\mathrm{c}_{\beta}^{3}}\left(\frac{\mathrm{s}_{\alpha} \mathrm{c}_{\beta-\alpha}}{m_{h}^{2}}+\frac{\mathrm{c}_{\alpha} \mathrm{s}_{\beta-\alpha}}{m_{H}^{2}} \pm \frac{\mathrm{s}_{\beta}}{m_{A}^{2}}\right) .
$$


The operators proportional to $\mathcal{C}_{+}$can be Fierz-rearranged and cast in a form already exhibited in eq. (19). In other words, these contributions can equivalently be regarded as a shift in $F_{L}^{\mu_{R}}$ and $F_{R}^{\mu_{L}}$, that is

$$
\delta F_{L}^{\mu_{R}}=\frac{G_{F}}{\sqrt{2}} m_{\mu} m_{\tau} \mathcal{C}_{+} \Delta_{R}, \quad \delta F_{R}^{\mu_{L}}=\frac{G_{F}}{\sqrt{2}} m_{\mu} m_{\tau} \mathcal{C}_{+} \Delta_{L},
$$

and the branching ratio (23) is accordingly affected. The operators proportional to $\mathcal{C}_{-}$have a different chiral structure and give an extra contribution $\left(m_{\mu} m_{\tau} \mathcal{C}_{-}\right)^{2}\left(\left|\Delta_{L}\right|^{2}+\right.$ $\left.\left|\Delta_{R}\right|^{2}\right) / 32$ to $B R(\tau \rightarrow 3 \mu) / B R(\tau \rightarrow \mu \nu \nu)$. Notice, however, that the Higgs-mediated contributions are potentially relevant only for large $\tan \beta$, so that the lepton mass suppression is overcome. More precisely, the truly enhanced operators are those proportional to $\mathcal{C}_{+}$, which becomes $\mathcal{C}_{+} \simeq 2 \tan ^{3} \beta / m_{A}^{2}$. In the limit in which the Higgs-mediated contribution dominates over the other ones (in particular, the dipole one), the decay $\tau \rightarrow 3 \mu$ is directly correlated to the decay $A \rightarrow \mu \tau[11]$ :

$$
B R\left(\tau^{-} \rightarrow \mu^{-} \mu^{+} \mu^{-}\right) \sim 4 \times 10^{-5}\left(\frac{\tan \beta}{40}\right)^{4}\left(\frac{100 \mathrm{GeV}}{m_{A}}\right)^{4} \frac{B R\left(A \rightarrow \mu^{+} \tau^{-}\right)}{B R\left(A \rightarrow \tau^{+} \tau^{-}\right)}
$$

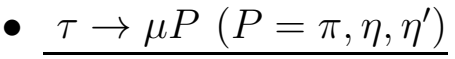

Now consider the implications of virtual Higgs exchange for the decays $\tau \rightarrow \mu P$, where $P$ is a neutral pseudoscalar meson $\left(P=\pi, \eta, \eta^{\prime}\right)$. Since we assume CP conservation in the Higgs sector, only the exchange of the $A$ Higgs boson is relevant. Moreover, in the large $\tan \beta$ limit, only the $A$ couplings to down-type quarks are important. These can be written as:

$$
-i\left(\sqrt{2} G_{F}\right)^{1 / 2} \tan \beta A\left(\xi_{d} m_{d} d^{c} d+\xi_{s} m_{s} s^{c} s+\xi_{b} m_{b} b^{c} b\right)+\text { h.c. }
$$

The parameters $\xi_{d}, \xi_{s}, \xi_{b}$ are equal to one at tree level, but can significantly deviate from this value because of higher order corrections proportional to $\tan \beta[23,24]$, generated by integrating out superpartners ${ }^{9}$. At energies below the bottom mass, the $b$-quark can be integrated out, so in eq. (61) the bilinear $-i m_{b} b^{c} b+$ h.c. is effectively replaced by the gluon operator $\Omega=\frac{g_{s}^{2}}{64 \pi^{2}} \epsilon^{\mu \nu \rho \sigma} G_{\mu \nu}^{a} G_{\rho \sigma}^{a}$, where $g_{s}$ and $G_{\mu \nu}^{a}$ are the $S U(3)_{C}$ coupling constant and field strength, respectively (see Fig. 4). The effective lagrangian due to $A$ boson exchange, relevant for $\tau \rightarrow \mu P\left(P=\pi, \eta, \eta^{\prime}\right)$, reads:

$$
\delta \mathcal{L}^{\mathrm{eff}}=i \sqrt{2} G_{F} m_{\tau} \frac{\tan ^{3} \beta}{m_{A}^{2}}\left[\xi_{d} m_{d} j_{d}^{5}+\xi_{s} m_{s} j_{s}^{5}+\xi_{b} \Omega\right]\left(\Delta_{R} \mu^{c} \tau+\Delta_{L}^{*} \mu \tau^{c}\right)+\text { h.c. },
$$

\footnotetext{
${ }^{9}$ In the limit of quark flavour conservation, each $\xi_{q}(q=d, s, b)$ has the form $\xi_{q}=\left(1+\Delta_{q} \tan \beta\right)^{-1}$, where $\Delta_{q}$ appears in the loop-generated term $-h_{q} \Delta_{q} H_{2}^{0 *} q^{c} q+$ h.c.. We recall that the leading contribution to $\Delta_{q}$ arises from a gluino-squark loop and reads $\Delta_{q} \simeq-\frac{2 \alpha_{s}}{3 \pi} \mu M_{3} I_{3}\left(M_{3}^{2}, \tilde{m}_{\tilde{q}_{L}}^{2}, \tilde{m}_{\tilde{q}_{R}}^{2}\right)$. A Higgsino-stop loop also contributes to $\Delta_{b}$ through a term $-\frac{\alpha_{t}}{4 \pi} \mu A_{t} I_{3}\left(\mu^{2}, \tilde{m}_{\tilde{t}_{L}}^{2}, \tilde{m}_{\tilde{t}_{R}}^{2}\right)$.
} 
where we have defined the quark pseudoscalar densities ${ }^{10} j_{q}^{5}=i\left(\bar{q} \bar{q}^{c}-q^{c} q\right)$, with $q=d, s$, and approximated $1 / \mathrm{c}_{\beta}^{2} \simeq \tan ^{2} \beta$. The matrix elements $\left\langle 0\left|j_{q}^{5}\right| P\right\rangle$ and $\langle 0|\Omega| P\rangle$ can be determined along the lines of [25], i.e. by taking the matrix elements of $\partial_{\mu} J_{5}^{\mu 3}, \partial_{\mu} J_{5}^{\mu 8}, \partial_{\mu} J_{5}^{\mu 0}$ (the latter divergence is anomalous and contains the term $-\sqrt{6} \Omega$ ). We find:

$$
\begin{aligned}
\left\langle 0\left|m_{d} j_{d}^{5}\right| \pi\right\rangle & =-\frac{f_{\pi} m_{\pi}^{2}}{1+z}, \quad & \left\langle 0\left|m_{s} j_{s}^{5}\right| \pi\right\rangle=\langle 0|\Omega| \pi\rangle=-\frac{1}{2}\left(\frac{1-z}{1+z}\right) f_{\pi} m_{\pi}^{2}, \\
\left\langle 0\left|m_{s} j_{s}^{5}\right| \eta\right\rangle & =-\frac{\sqrt{3}}{2} f_{\eta}^{8} m_{\eta}^{2}, & \langle 0|\Omega| \eta\rangle=-\frac{1}{\sqrt{6}}\left(\frac{1}{\sqrt{2}} f_{\eta}^{8}+f_{\eta}^{0}\right) m_{\eta}^{2}, \\
\left\langle 0\left|m_{s} j_{s}^{5}\right| \eta^{\prime}\right\rangle & =-\frac{\sqrt{3}}{2} f_{\eta^{\prime}}^{8} m_{\eta^{\prime}}^{2}, & \left\langle 0|\Omega| \eta^{\prime}\right\rangle=-\frac{1}{\sqrt{6}}\left(f_{\eta^{\prime}}^{0}+\frac{1}{\sqrt{2}} f_{\eta^{\prime}}^{8}\right) m_{\eta^{\prime}}^{2},
\end{aligned}
$$

where $z=m_{u} / m_{d}$. In deriving the matrix elements with $\eta, \eta^{\prime}$ we have neglected $m_{u}, m_{d}$. All these results translate into additional contributions to the effective meson lagrangians $\mathcal{L}_{\tau \mu \pi}^{\text {eff }}, \mathcal{L}_{\tau \mu \eta}^{\text {eff }}, \mathcal{L}_{\tau \mu \eta^{\prime}}^{\text {eff }}$ in Section 2.2. Such contributions can be interpreted as shifts in $F_{L(R)}^{\pi}$, $F_{L(R)}^{\eta, 8}, F_{L(R)}^{\eta, 0}, F_{L(R)}^{\eta^{\prime}, 8}, F_{L(R)}^{\eta^{\prime}, 0}$, that is

$$
\begin{aligned}
\delta F_{L(R)}^{\pi} & =-\sqrt{2} G_{F} m_{\pi}^{2}\left(\xi_{d} \frac{1}{1+z}+\frac{\xi_{s}+\xi_{b}}{2} \frac{1-z}{1+z}\right) \frac{\tan ^{3} \beta}{m_{A}^{2}} \Delta_{L(R)}, \\
\delta F_{L(R)}^{\eta, 8} & =-\sqrt{\frac{3}{2}} G_{F} m_{\eta}^{2}\left(\xi_{s}+\frac{1}{3} \xi_{b}\right) \frac{\tan ^{3} \beta}{m_{A}^{2}} \Delta_{L(R)} \\
\delta F_{L(R)}^{\eta, 0} & =-\frac{1}{\sqrt{3}} G_{F} m_{\eta}^{2} \xi_{b} \frac{\tan ^{3} \beta}{m_{A}^{2}} \Delta_{L(R)}
\end{aligned}
$$

and $\delta F_{L(R)}^{\eta^{\prime}, 8}, \delta F_{L(R)}^{\eta^{\prime}, 0}$ can be obtained from $\delta F_{L(R)}^{\eta, 8}, \delta F_{L(R)}^{\eta, 0}$ by the substitution $\eta \rightarrow \eta^{\prime}$. We have performed a cross-check by using the approach of chiral perturbation theory and obtained results that are consistent with those above.

In the limit in which the processes $\tau \rightarrow 3 \mu$ and $\tau \rightarrow \mu \eta$ are both dominated by Higgsexchange, these decays are related as:

$$
\frac{B R\left(\tau^{-} \rightarrow \mu^{-} \eta\right)}{B R\left(\tau^{-} \rightarrow \mu^{-} \mu^{+} \mu^{-}\right)} \simeq 36 \pi^{2}\left(\frac{f_{\eta}^{8} m_{\eta}^{2}}{m_{\mu} m_{\tau}^{2}}\right)^{2}\left(1-x_{\eta}\right)^{2}\left[\xi_{s}+\frac{\xi_{b}}{3}\left(1+\sqrt{2} \frac{f_{\eta}^{0}}{f_{\eta}^{8}}\right)\right]^{2}
$$

For $\xi_{s} \sim \xi_{b} \sim 1$ this ratio is about 5 , but it could also be a few times larger or smaller than that, depending on the actual values of $\xi_{s}, \xi_{b}$. Our result confirms the relevance of Higgs-exchange in $\tau \rightarrow \mu \eta$, previously emphasized in [21]. In the latter paper, however, neither the contribution of the (bottom-loop induced) gluon operator $\Omega$ nor the factors $\xi_{q}$ were included. Moreover, if these effects were disregarded, the ratio (69) would be 3 times smaller than the one found in [21] (notice that $F_{\eta}^{8}$ in [21] corresponds to $\sqrt{2} f_{\eta}^{8}$ ).

\footnotetext{
${ }^{10}$ In four-component notation the pseudoscalar densities read as $j_{q}^{5}=i \bar{\psi}_{q} \gamma^{5} \psi_{q}$ with $\psi_{q}^{T}=\left(\begin{array}{ll}q & \bar{q}^{c}\end{array}\right)$.
} 
Finally, let us compare $\tau \rightarrow \mu \eta^{\prime}$ and $\tau \rightarrow \mu \pi$ with $\tau \rightarrow \mu \eta$ in the limit of Higgs-exchange domination:

$$
\begin{aligned}
& \frac{B R\left(\tau^{-} \rightarrow \mu^{-} \eta^{\prime}\right)}{B R\left(\tau^{-} \rightarrow \mu^{-} \eta\right)} \simeq \frac{2}{9}\left(\frac{f_{\eta^{\prime}}^{0}}{f_{\eta}^{8}}\right)^{2} \frac{m_{\eta^{\prime}}^{4}}{m_{\eta}^{4}}\left(\frac{1-x_{\eta^{\prime}}}{1-x_{\eta}}\right)^{2}\left[\frac{1+\frac{3}{\sqrt{2}} \frac{f_{\eta^{\prime}}^{8}}{f_{\eta^{\prime}}^{0}}\left(\frac{\xi_{s}}{\xi_{b}}+\frac{1}{3}\right)}{\frac{\xi_{s}}{\xi_{b}}+\frac{1}{3}+\frac{\sqrt{2}}{3} \frac{f_{\eta}^{0}}{f_{\eta}^{8}}}\right]^{2}, \\
& \frac{B R\left(\tau^{-} \rightarrow \mu^{-} \pi\right)}{B R\left(\tau^{-} \rightarrow \mu^{-} \eta\right)} \simeq \frac{4}{3}\left(\frac{f_{\pi}}{f_{\eta}^{8}}\right)^{2} \frac{m_{\pi}^{4}}{m_{\eta}^{4}}\left(1-x_{\eta}\right)^{-2}\left[\frac{\frac{\xi_{d}}{\xi_{b}} \frac{1}{1+z}+\frac{1}{2}\left(1+\frac{\xi_{s}}{\xi_{b}}\right) \frac{1-z}{1+z}}{\frac{\xi_{s}}{\xi_{b}}+\frac{1}{3}+\frac{\sqrt{2}}{3} \frac{f_{\eta}^{0}}{f_{\eta}^{8}}}\right]^{2} .
\end{aligned}
$$

Both ratios are suppressed, although for different reasons. The ratio $B R\left(\tau \rightarrow \mu \eta^{\prime}\right) / B R(\tau \rightarrow$ $\mu \eta)$, which seems to be $\mathcal{O}(1)$, is much smaller because the singlet and octet contributions to $\tau \rightarrow \mu \eta^{\prime}$ tend to cancel against each other (we recall that $f_{\eta^{\prime}}^{8} / f_{\eta^{\prime}}^{0} \sim-0.4$ ). For $\xi_{s} / \xi_{b} \sim 1$, the ratio $(70)$ is $6 \times 10^{-3}$. The ratio $B R(\tau \rightarrow \mu \pi) / B R(\tau \rightarrow \mu \eta)$ is small because it is parametrically suppressed by $m_{\pi}^{4} / m_{\eta}^{4} \sim 10^{-2}$. The actual numerical value is sensitive to the parameter $z=m_{u} / m_{d}$, and to the ratios $\xi_{d} / \xi_{b}, \xi_{s} / \xi_{b}$. In the case $\xi_{d} / \xi_{b} \sim \xi_{s} / \xi_{b} \sim 1$, if we let $z$ vary between 0.2 and 0.7 [7] the ratio (71) ranges from $4 \times 10^{-3}$ to $10^{-3}$. These results, combined with the present bound (6) on $\tau \rightarrow \mu \eta$, imply that the Higgs mediated contribution to $B R\left(\tau \rightarrow \mu \eta^{\prime}\right)$ and $B R(\tau \rightarrow \mu \pi)$ can reach $\mathcal{O}\left(10^{-9}\right)$.

\subsection{Muon anomalous magnetic moment}

The flavour changing dipole operators in eq. (13) have an obvious flavour conserving counterpart, which for the muon is

$$
e m_{\mu} i D_{\mu}^{\gamma} \mu^{c} \sigma^{\rho \sigma} \mu F_{\rho \sigma}+\text { h.c. }
$$

The coefficient $D_{\mu}^{\gamma}$ is directly related to the anomalous magnetic moment of the muon: $a_{\mu} \equiv$ $\left(g_{\mu}-2\right) / 2=2 m_{\mu}^{2} \operatorname{Re} D_{\mu}^{\gamma}$. In the MSSM the coefficient $D_{\mu}^{\gamma}$ receives three types of one-loop contributions from superparticle exchange, analogous to those shown in Fig. 2. In turn the (a)type and (b)-type contributions can be further distinguished according to the chirality (either $L$ or $R$ ) of the sleptons circulating in the loop. In the (c)-type contributions both $L$ and $R$ sleptons are simultaneously present. Therefore we can express the superparticle contribution to $a_{\mu}$, denoted as $a_{\mu}^{\mathrm{MSSM}}$, as the sum of five terms:

$$
a_{\mu}^{\mathrm{MSSM}}=a_{\mu L}^{(a)}+a_{\mu R}^{(a)}+a_{\mu L}^{(b)}+a_{\mu R}^{(b)}+a_{\mu L R}^{(c)} .
$$

Non-vanishing contributions to $a_{\mu}^{\mathrm{MSSM}}$ arise even in the absence of LFV, of course [26, 27]. In this case the only sleptons involved are $\tilde{L}_{\mu}=\left(\tilde{\nu}_{\mu}, \tilde{\mu}_{L}\right)$ and $\tilde{\mu}_{R}$, and the contributions to $a_{\mu}$ are generically proportional to $\left(g_{w}^{2} / 16 \pi^{2}\right)\left(m_{\mu}^{2} / M_{S}^{2}\right)$, possibly with an extra $\tan \beta$ factor. In the case of mixing between second and third slepton generations the no-mixing results need to be generalized (see Appendix A.10). One of the effects of LFV is that (c)-type contributions have extra terms proportional to $m_{\tau} / m_{\mu}$, as already observed in [27, 28]. These contributions are potentially the largest ones if $(\mathrm{LFV})_{L}$ and $(\mathrm{LFV})_{R}$ are both large. However, the latter situation does not seem very natural if the smallness of $m_{\mu} / m_{\tau}$ is to be explained by an underlying 
flavour symmetry. In fact, we rather expect LFV to be large in at most one sector $(L$ or $R)$. In such a case the apparent $m_{\tau} / m_{\mu}$-enhancement is compensated by suppressing factors, so that the size of such extra contributions to $a_{\mu}^{\mathrm{MSSM}}$ does not exceed that of the other ones.

We also recall that, at present, it is not clear whether a discrepancy exists between the experimental determination of $a_{\mu}$ and the Standard Model prediction (see e.g. [29]). Hence some caution is needed in deriving constraints on the MSSM parameter space [30]. 


\section{$4 \quad$ Large $(\mathrm{LFV})_{L}:$ numerical analysis}

In this section we perform a detailed numerical analysis in the case of large $(\mathrm{LFV})_{L}$, assuming vanishing $(\mathrm{LFV})_{R}$, i.e. $\tilde{m}_{R \mu \tau}^{2}=A_{\mu \tau}^{R}=0$. We remark that all operator coefficients depend on $\tilde{m}_{L_{\alpha}}, \theta_{L}$ and gaugino masses. Some coefficients also depend on additional parameters. In particular, $A_{L}^{Z(a)}$ and $D_{L}^{\gamma(b)}$ depend on $\mu$ and $\beta ; D_{L}^{\gamma(c)}$ depends on $\mu, \beta, \tilde{m}_{\tilde{\tau}_{R}}, A_{\tau}, A_{\mu \tau}^{L} ; B_{L}^{f}$ depends on $\tilde{m}_{\tilde{f}}$.

The lightest eigenvalue of $\tilde{\mathcal{M}}_{L}^{2}$ is conventionally chosen to be $\tilde{m}_{L_{3}}^{2}$ (although our formulae in Appendix do not depend on such a choice). To enhance $(\mathrm{LFV})_{L}$, in all our numerical examples we will take maximal mixing, $\theta_{L}=\pi / 4$ (i.e. $\tilde{m}_{L \mu \mu}^{2}=\tilde{m}_{L \tau \tau}^{2}$ in $\tilde{\mathcal{M}}_{L}^{2}$ ), and widely split eigenvalues. The mass parameters will be varied in such a way that charged sparticle masses be $\gtrsim 100 \mathrm{GeV}$, to respect the LEP constraints [7,31]. We will also check that neutralino production at LEP be either kinematically forbidden or adequately suppressed [32]. Incidentally, we should add that we have performed such checks using the full mass eigenvalues (not just those at zero-th order in electroweak breaking). For simplicity, we take a common (soft-breaking) mass $\tilde{m}_{\tilde{e}}$ for selectrons of both chiralities, as well as a common mass $\tilde{m}_{\tilde{q}}$ for first and second generation squarks of both chiralities.

\section{1 $(\text { LFV })_{L}$ with small $\tan \beta$}

The decay $\tau \rightarrow \mu \gamma$ poses significant constraints on the MSSM parameter space, especially for large $(\mathrm{LFV})_{L}$. Moreover, the decays $\tau \rightarrow \mu e e, \tau \rightarrow \mu \mu \mu, \tau \rightarrow \mu \rho$ are often dominated by the dipole contribution. One of our purposes is to study to which extent the latter property holds in the MSSM parameter space or, more generally, to study the interplay between dipole and non-dipole contributions. We start our numerical analysis by considering in more detail the contributions to the dipole operator, taking into account that the present bound (1) on $\tau \rightarrow \mu \gamma$ translates into the bound $\left|D_{L}^{\gamma}\right| \lesssim 5 \times 10^{-9} \mathrm{GeV}^{-2}$.
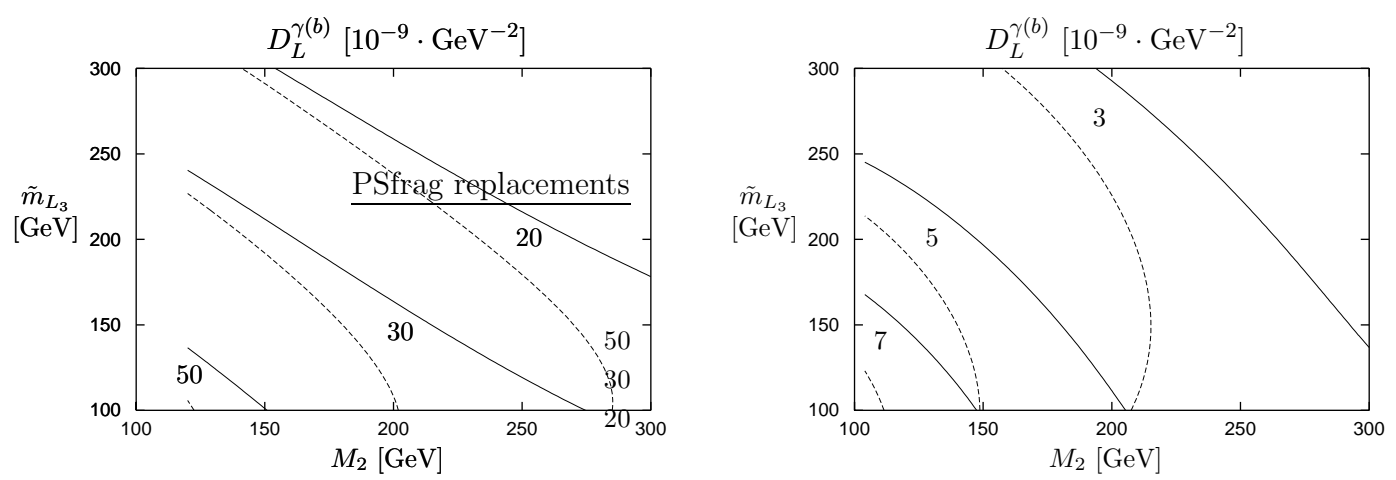

Figure 5: $D_{L}^{\gamma(b)}$ isocontours with $\tan \beta=3, \tilde{m}_{L_{2}}=1 \mathrm{TeV}, \theta_{L}=\pi / 4$ and $\mu=140 \mathrm{GeV}(1 \mathrm{TeV})$ in the left (right) panel. The solid and dashed lines refer to $M_{1}=100 \mathrm{GeV}$ and $M_{1}=-100 \mathrm{GeV}$, respectively.

The coefficient $\left|D_{L}^{\gamma(a)}\right|$ is at most $10^{-9} \mathrm{GeV}^{-2}$, so it fulfils the bound. In contrast, $D_{L}^{\gamma(b)}$ and $D_{L}^{\gamma(c)}$ may separately exceed the bound in some regions of the parameter space, especially 
for large $\tan \beta$. In this section we focus on small $\tan \beta$. Incidentally, notice that monopole coefficients do not have a strong $\tan \beta$ dependence. In Fig. 5 we show the contours of the coefficient $D_{L}^{\gamma(b)}$ in the plane $\left(M_{2}, \tilde{m}_{L_{3}}\right)$ with $\tan \beta=3, \theta_{L}=\pi / 4, \tilde{m}_{L_{2}}=1 \mathrm{TeV},\left|M_{1}\right|=$ $100 \mathrm{GeV}$ and $\mu=140 \mathrm{GeV}(1 \mathrm{TeV})$ in the left (right) panel. For the range of $M_{2}$ and $\tilde{m}_{L_{3}}$ shown in the figure, $D_{L}^{\gamma(b)}$ is well above the bound for small $\mu$, whilst it is of the same order of the bound for large $\mu$. Also $D_{L}^{\gamma(c)}$ may or may not exceed the bound, depending on the range of the extra parameters $\tilde{m}_{\tilde{\tau}_{R}}, A_{\tau}, A_{\mu \tau}^{L}$. Even if $D_{L}^{\gamma(b)}$ and $D_{L}^{\gamma(c)}$ should separately exceed the bound, however, mutual cancellations could bring the total dipole contribution $D_{L}^{\gamma}$ below the bound. To illustrate this, in Fig. 6 we depict the $D_{L}^{\gamma}$ contours in the plane $\left(A_{\tau}, \tilde{m}_{\tilde{\tau}_{R}}\right)$ for

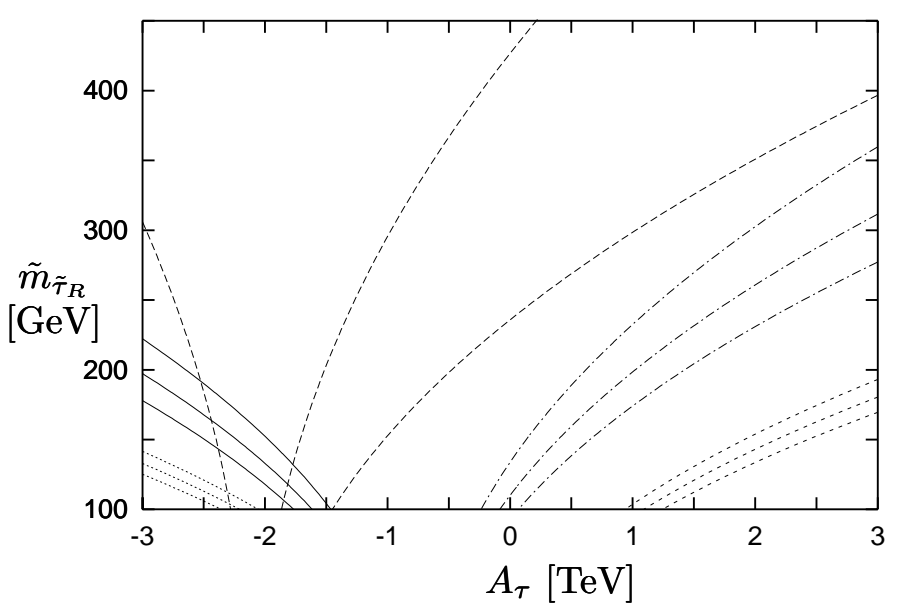

Figure 6: $D_{L}^{\gamma}$ contours for $\tan \beta=3, \tilde{m}_{L_{2}}=1 \mathrm{TeV}, \theta_{L}=\pi / 4, A_{\mu \tau}^{L}=0$ and five choices of $\left(\tilde{m}_{L_{3}}, \mu, M_{2}, M_{1}\right)$ in GeV: $(100,140,120,100)$ (dotted), $(100,140,120,-100)$ (short-dashed), $(100,250,150,100)$ (solid), (100, 250, 150,-100) (dot-dashed), (200,700,300,-100) (dashed). For each example the two external lines correspond to $\left|D_{L}^{\gamma}\right|=5 \times 10^{-9} \mathrm{GeV}^{-2}$ and the middle one to $D_{L}^{\gamma}=0$.

$\tan \beta=3, \tilde{m}_{L_{2}}=1 \mathrm{TeV}, A_{\mu \tau}^{L}=0$ and several choices of $\left(\tilde{m}_{L_{3}}, \mu, M_{1}, M_{2}\right)$. For each case we show three lines: the two external ones delimit the allowed region $\left(\left|D_{L}^{\gamma}\right| \leq 5 \times 10^{-9} \mathrm{GeV}^{-2}\right)$, the middle one corresponds to full cancellation $\left(D_{L}^{\gamma}=0\right)$. The coefficient $D_{L}^{\gamma(b)}$ is above the bound in the four cases with small $\mu$, while it is below in the example with large $\mu$, hence in the latter case the allowed region is wider. In the examples with $M_{1}>0$, large and negative $A_{\tau}$ values ${ }^{11}$ are required to make the sign of $D_{L}^{\gamma(c)}$ opposite to that of $D_{L}^{\gamma(b)}$. We also note that, in general, the role of $A_{\tau}$ would be shared by $A_{\tau}$ and $A_{\mu \tau}^{L}$ [see eq. (105)].

A remark is in order about the muon anomalous magnetic moment. There are obvious analogies between the contributions to $D_{\mu}^{\gamma}$ (i.e. to $\left.a_{\mu}^{\mathrm{MSSM}}\right)$ and those to $D^{\gamma}$, such as a similar diagrammatic origin and the dependence on common parameters (see Appendices A.6 and A.10). In the

\footnotetext{
${ }^{11}$ A situation with $\left|A_{\tau}\right| \gg \tilde{m}_{L_{3}}, \tilde{m}_{\tilde{\tau}_{R}}$ could destabilize the scalar potential and induce VEVs for slepton fields. To avoid this one can take, for instance, a sufficiently large $m_{A}$. The inequality $m_{\tau}\left|A_{\tau}+\mu \tan \beta\right|<\sqrt{2} \tilde{m}_{L_{3}} \tilde{m}_{\tilde{\tau}_{R}}$ is another requirement to avoid tachyonic sleptons (here $\theta_{L}=\pi / 4$ ). This has been verified throughout all our numerical examples. Similar considerations apply to the $(\mathrm{LFV})_{R}$ case in Section 5 , with $L \leftrightarrow R$.
} 

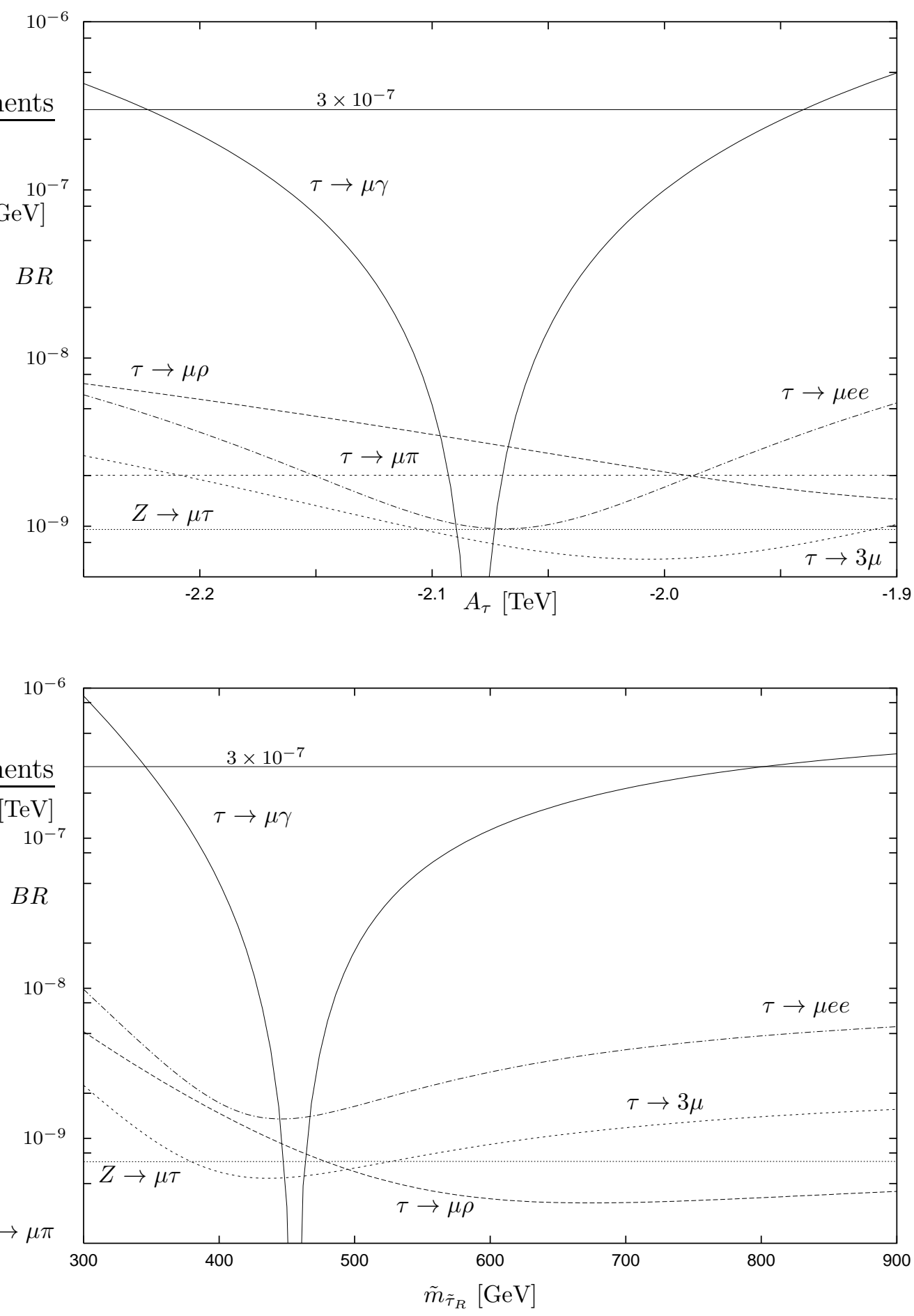

Figure 7: Branching ratios of LFV decays for $\tan \beta=3, \tilde{m}_{L_{2}}=1 \mathrm{TeV}, \tilde{m}_{L_{3}}=100 \mathrm{GeV}, \theta_{L}=\pi / 4$, $A_{\mu \tau}^{L}=0$. In the upper panel, the remaining parameters are: $\mu=120 \mathrm{GeV}, M_{2}=150 \mathrm{GeV}, M_{1}=100 \mathrm{GeV}$, $\tilde{m}_{\tilde{\tau}_{R}}=\tilde{m}_{\tilde{\mu}_{R}}=100 \mathrm{GeV}, \tilde{m}_{\tilde{e}}=\tilde{m}_{\tilde{q}}=1 \mathrm{TeV}$. In the lower panel: $\mu=800 \mathrm{GeV}, M_{2}=120 \mathrm{GeV}, M_{1}=-100 \mathrm{GeV}$, $A_{\tau}=0, \tilde{m}_{\tilde{e}}=120 \mathrm{GeV}, \tilde{m}_{\tilde{q}}=1 \mathrm{TeV}$ and $\tilde{m}_{\tilde{\mu}_{R}}=\tilde{m}_{\tilde{\tau}_{R}}$. The solid horizontal line indicates the present bound on $B R(\tau \rightarrow \mu \gamma)$. In the upper example: $B R(\tau \rightarrow \mu \eta) \sim 6 \times 10^{-10}, B R\left(\tau \rightarrow \mu \eta^{\prime}\right) \sim 5 \times 10^{-10}$. In the lower example: $B R(\tau \rightarrow \mu \pi) \sim 2 \times 10^{-12}, B R(\tau \rightarrow \mu \eta) \sim B R\left(\tau \rightarrow \mu \eta^{\prime}\right) \sim 10^{-12}$. 
case of large $(\mathrm{LFV})_{L}$, for instance, we have typically that $a_{\mu L}^{(a, b)} \sim-0.02 \times\left(D_{L}^{\gamma(a, b)} / \mathrm{GeV}^{-2}\right)$. So if $D_{L}^{\gamma(b)}$ saturates the present bound on $D^{\gamma}$, then $\left|a_{\mu L}^{(b)}\right| \lesssim 10^{-10}$, while if $D_{L}^{\gamma(b)}$ is ten times larger than the bound (which should be fulfilled through cancellations), then $\left|a_{\mu L}^{(b)}\right| \sim 10^{-9}$. This is of the order of the experimental error on $a_{\mu}$ and could either be acceptable on its own or play a role in explaining a possible discrepancy between the experimental determination of $a_{\mu}$ and the Standard Model prediction. The connection between other contributions to $a_{\mu}^{\mathrm{MSSM}}$ and $D_{L}^{\gamma}$ is less direct, since some parameters differently affect each of them. For instance, in the $(\mathrm{LFV})_{L}$ case that we are considering here, $a_{\mu}^{\mathrm{MSSM}}$ depends also on $\tilde{m}_{\tilde{\mu}_{R}}$ and $A_{\mu}$, which do not enter the $D_{L}^{\gamma}$-coefficients (at least at leading order). In particular, cancellations among different contributions to $a_{\mu}^{\mathrm{MSSM}}$ (analogous to those in $D_{L}^{\gamma}$ ) may or may not occur, depending on the choice of such parameters. All such features loosen the correlation between $a_{\mu}^{\mathrm{MSSM}}$ and $D^{\gamma}$. Since we work in an unconstrained MSSM framework, we content ourselves with verifying that the numerical examples of this Section are consistent with values of $\left|a_{\mu}^{\mathrm{MSSM}}\right|$ of order $10^{-9}$ or smaller. Similar considerations apply to the $(\mathrm{LFV})_{R}$ case discussed in Section 5 (where we shall not come back to this point).

After this digression on $a_{\mu}$, let us continue the discussion on the LFV operators. It is interesting to see how the branching ratios of the different LFV processes behave when the $D_{L}^{\gamma}$ dipole contribution varies and the monopole ones are basically fixed. In Fig. 7 we plot two such examples, where $\mu$ is either small (upper panel) or large (lower panel). The specific values of the parameters involved are given in the caption. The parameter that varies on the horizontal axis (either $A_{\tau}$ or $\tilde{m}_{\tilde{\tau}_{R}}$ ) has the only role to induce a variation of the $D_{L}^{\gamma}$ dipole contribution. The $B R \mathrm{~s}$ of $Z \rightarrow \mu \tau, \tau \rightarrow \mu \pi, \tau \rightarrow \mu \eta, \tau \rightarrow \mu \eta^{\prime}$ are constant ${ }^{12}$. In the other decays we can observe an interplay between the dipole and monopole contributions, which are comparable in magnitude in these examples. For instance, in both examples the pure monopole contribution to $B R(\tau \rightarrow \mu e e)$ amounts to about $10^{-9}$, as we can read in correspondence of the dipole cancellation $(\tau \rightarrow \mu \gamma$ 'dip'). At the points where $\tau \rightarrow \mu \gamma$ saturates the bound, the pure dipole contribution is $3 \times 10^{-9}$ [see eq. (37)] and the combined contribution can reach $5 \times 10^{-9}$. In the case of $\tau \rightarrow \mu \rho$ we can also notice a strong interference effect between the dipole and the monopole contributions. Consider, for instance, the first example. In correspondence of the $\tau \rightarrow \mu \gamma$-saturation points, $B R(\tau \rightarrow \mu \rho)$ is about $6 \times 10^{-9}$ (left point) and $1.5 \times 10^{-9}$ (right point). These numbers should be compared with the pure dipole contribution at those points, $0.8 \times 10^{-9}$ [eq. (39)], and the pure monopole one, $3 \times 10^{-9}$.

The previous examples have shown that monopole operators can play an important role. We recall that the monopole coefficients $\left(F^{Z}, F^{\mu}, F^{e}, F^{\rho}, F^{\pi}, F^{\eta}, F^{\eta^{\prime}}\right)$ receive different contributions (from $A^{Z}, C^{Z}, C^{\gamma}, B^{f}$ ), which have specific parameter dependences. Notice that all these contributions depend on $\tilde{m}_{L_{3}}$ and $M_{2}$, so latter masses should not be too large, if we want to avoid a strong suppression of monopole coefficients. The relevance of each contribution, as well as the possibility of mutual cancellations, is also influenced by other parameters, such as $\mu$ (in $A^{Z}$ ), the selectron masses (in $B^{e}$ ) or the squark masses (in $B^{u}, B^{d}, B^{s}$ ). In the first example of

\footnotetext{
${ }^{12}$ In fact, $B R(Z \rightarrow \mu \tau)$ depends on $\tilde{m}_{\tilde{\tau}_{R}}$ through the coefficients $A_{L}^{Z(b, c)}, D_{L}^{Z(b, c)}$. Similarly, $B R(\tau \rightarrow$ $\mu \pi), B R(\tau \rightarrow \mu \eta)$ and $B R\left(\tau \rightarrow \mu \eta^{\prime}\right)$ depend on $\tilde{m}_{\tilde{\tau}_{R}}$ through the coefficients $A_{L}^{Z(b, c)}, \Delta_{L}^{(c)}$. However, for small $\tan \beta$ these contributions are suppressed.
} 
Fig. 7, for instance, the large values of $\tilde{m}_{\tilde{e}}$ and $\tilde{m}_{\tilde{q}}$ imply suppressed box contributions, while small $\mu$ implies unsuppressed $A^{Z}$-contribution (which combines with those of $C^{Z}$ or $C^{\gamma}$ ). In the second example, $A^{Z}$ is suppressed by the large $\mu$ value, whereas $B^{e}$ is unsuppressed because $\tilde{m}_{\tilde{e}}$ is small. In the following we shall analyse in more detail the parameter dependence of monopole contributions, considering one process at a time.
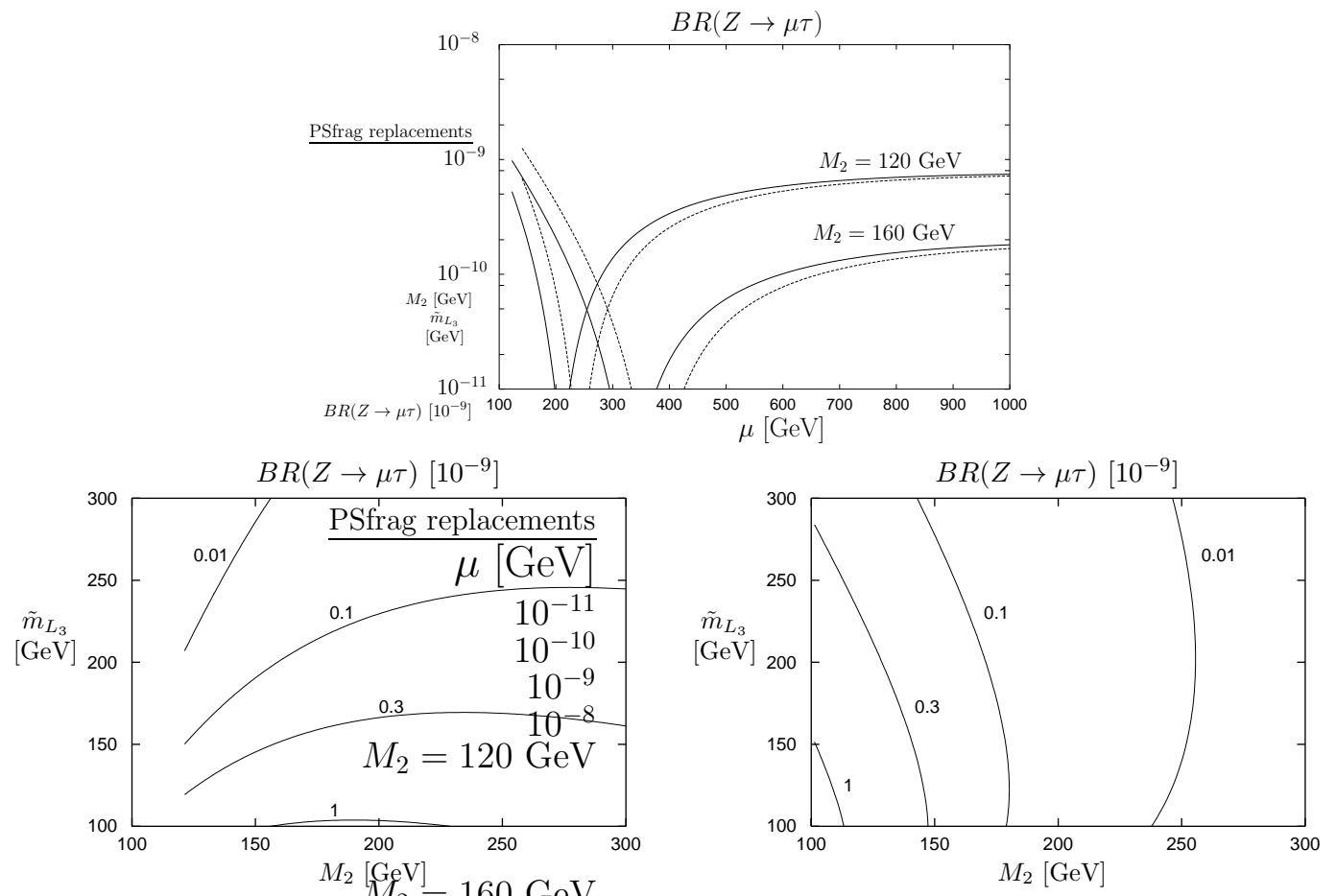

Figure 8: $B R(Z \rightarrow \mu \tau)$ for $\tan \beta=3, \tilde{m}_{L_{2}}=1 \mathrm{TeV}, \theta_{L}=\pi / 4$. In the upper panel: $\tilde{m}_{L_{3}}=100 \mathrm{GeV}, \tilde{m}_{\tilde{\tau}_{R}}=$ $100 \mathrm{GeV}, M_{2}$ as shown, $M_{1}=100(-100) \mathrm{GeV}$ for the solid (dashed) lines. In the left (right) lower panel: $\mu=120(1000) \mathrm{GeV}, M_{1}=100(-100) \mathrm{GeV}$ and $\tilde{m}_{\tilde{\tau}_{R}}=100(500) \mathrm{GeV}$.

In Fig. $8 B R(Z \rightarrow \mu \tau)$ is studied ${ }^{13}$ as a function of $\mu$ (upper panel) and in the $\left(M_{2}, \tilde{m}_{L_{3}}\right)$ plane for small $\mu$ ( left lower panel) or large $\mu$ (right lower panel). The behaviour in the upper panel shows the destructive interference of the $A^{Z}$ and $C^{Z}$ contributions. For small $\mu$ we have $\left|A_{L}^{Z}\right|>\left|C_{L}^{Z}\right|$. For large $\mu, A_{L}^{Z}$ is suppressed and $B R(Z \rightarrow \mu \tau)$ approaches an asymptotic value essentially determined by $C_{L}^{Z}$. The lower panels also show that $B R(Z \rightarrow \mu \tau)$ depends on $\left(M_{2}, \tilde{m}_{L_{3}}\right)$ in a different way for small and large $\mu$. In either case, this $B R$ can reach $\sim 10^{-9}$.

In Fig. 9 the monopole contribution to $B R(\tau \rightarrow \mu \mu \mu)$ is drawn as a function of $\mu$ (upper panel) and in the $\left(M_{2}, \tilde{m}_{L_{3}}\right)$ plane for small $\mu$ (left lower panel) or large $\mu$ (right lower panel). The monopole coefficients $F_{L}^{\mu_{L(R)}}$ receive contributions of different signs from $A_{L}^{Z}, C_{L}^{\gamma}$ and $B_{L}^{\mu_{L(R)}}$. The upper panel shows the changes in the interference pattern, since $A_{L}^{Z}$ is substantial for small $\mu$ and suppressed for large $\mu$, while $C_{L}^{\gamma}$ and $B_{L}^{\mu_{L(R)}}$ are constant. The lower panels show how the monopole-induced $B R(\tau \rightarrow \mu \mu \mu)$ depends on $\left(M_{2}, \tilde{m}_{L_{3}}\right)$ for small and large $\mu$. We can notice

\footnotetext{
${ }^{13}$ For earlier studies on $Z \rightarrow \mu \tau$ in the MSSM, see e.g. [33]. A more recent computation, with related discussion, appeared in [34].
} 


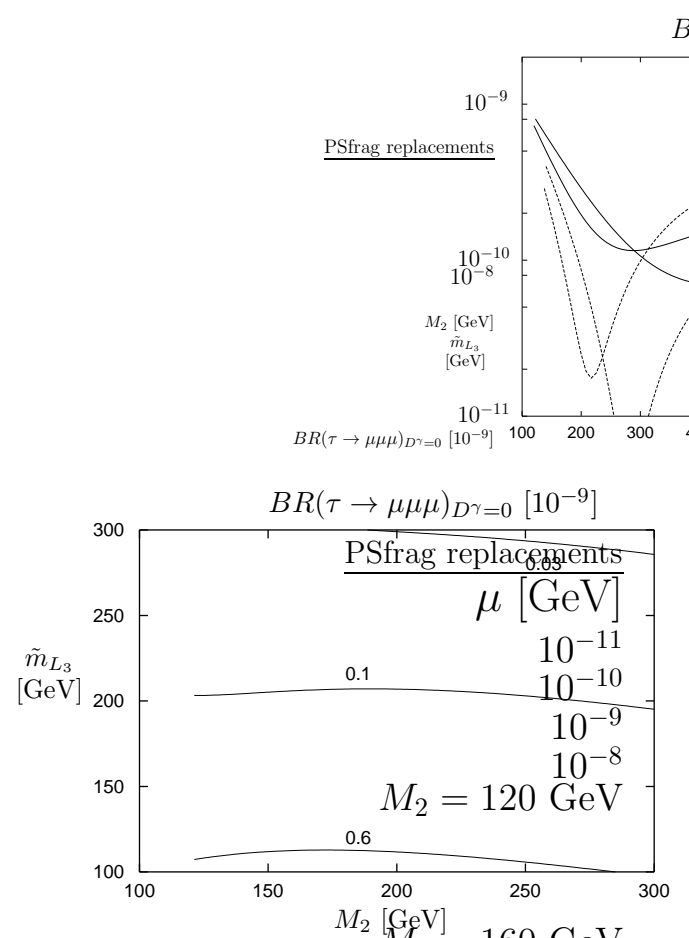

$B R(\tau \rightarrow \mu \mu \mu)_{D^{\gamma=0}}$

Figure 9: Monopole contribution to $B R(\tau \rightarrow \mu \mu \mu)$ for $\tan \beta=3, \tilde{m}_{L_{2}}=1 \mathrm{TeV}, \theta_{L}=\pi / 4$. In the upper panel: $\tilde{m}_{L_{3}}=100 \mathrm{GeV}, \tilde{m}_{\tilde{\tau}_{R}}=\tilde{m}_{\tilde{\mu}_{R}}=100 \mathrm{GeV}, M_{2}$ as shown, $M_{1}=100(-100) \mathrm{GeV}$ for the solid (dashed) lines. In the left (right) lower panel: $\mu=120(1000) \mathrm{GeV}, M_{1}=100(-100) \mathrm{GeV}$ and $\tilde{m}_{\tilde{\tau}_{R}}=100$ (500) GeV.
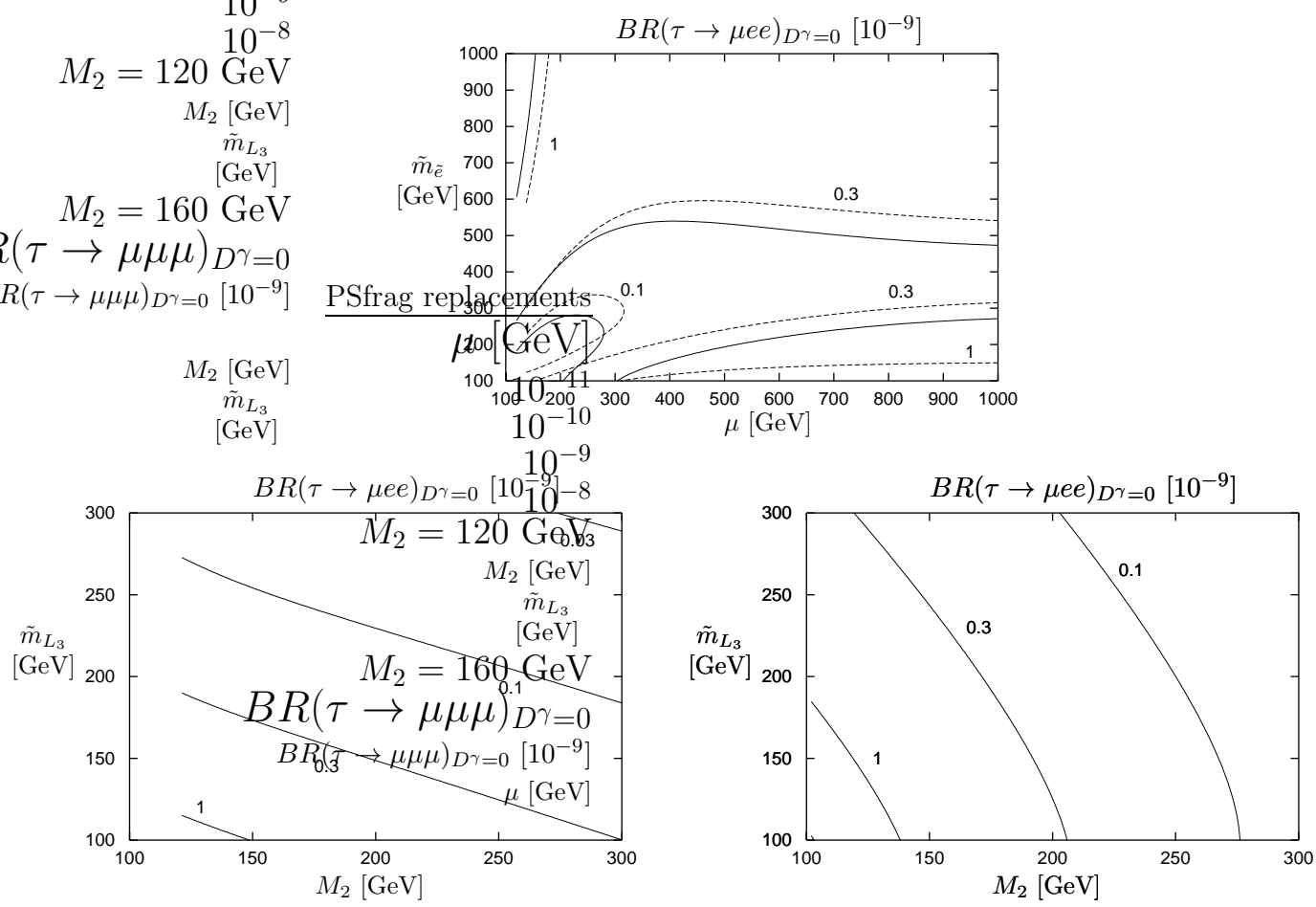

Figure 10: Monopole contribution to $B R(\tau \rightarrow \mu e e)$ for $\tan \beta=3, \tilde{m}_{L_{2}}=1 \mathrm{TeV}, \theta_{L}=\pi / 4$. In the upper panel: $\tilde{m}_{L_{3}}=100 \mathrm{GeV}, \tilde{m}_{\tilde{\tau}_{R}}=100 \mathrm{GeV}, M_{2}=120 \mathrm{GeV}, M_{1}=100(-100) \mathrm{GeV}$ for the solid (dashed) lines. In the left (right) lower panel: $\mu=120(1000) \mathrm{GeV}, M_{1}=100(-100) \mathrm{GeV}, \tilde{m}_{\tilde{e}}=1000$ (120) $\mathrm{GeV}$ and $\tilde{m}_{\tilde{\tau}_{R}}=100(500) \mathrm{GeV}$. 
that the maximal monopole contribution to $B R(\tau \rightarrow \mu \mu \mu)$ in these examples is comparable to the maximal allowed dipole contribution [eq. (38)], so the combination can be around $10^{-9}$ (see also Fig. 7).

In Fig. 10 the monopole contribution to $B R(\tau \rightarrow \mu e e)$ is plotted in the $\left(\mu, \tilde{m}_{\tilde{e}}\right)$ plane (upper panel) and in the $\left(M_{2}, \tilde{m}_{L_{3}}\right)$ plane for small $\mu$ and large $\tilde{m}_{\tilde{e}}$ (left lower panel) or large $\mu$ and small $\tilde{m}_{\tilde{e}}$ (right lower panel). The contours in the upper panel reflect the interference effects among the different contributions to $F_{L}^{e_{L(R)}}$, i.e. $A_{L}^{Z}, C_{L}^{\gamma}$ and $B_{L}^{e_{L(R)}}$. The monopole-induced $B R(\tau \rightarrow \mu e e)$ can be $\mathcal{O}\left(10^{-9}\right)$ either for small $\mu$ and large $\tilde{m}_{\tilde{e}}$ or for large $\mu$ and small $\tilde{m}_{\tilde{e}}$. In the former case $A_{L}^{Z}$ is substantial and box contributions are suppressed, in the latter case the opposite situation occurs. In both cases there is the constant $C^{\gamma}$ contribution. In other regions the $B R$ is smaller than $10^{-9}$ because of mutual cancellations. The maximal monopole contribution in these examples is comparable to the maximal allowed dipole contribution [eq. (37)], and the combination can be a few times $10^{-9}$ (see also Fig. 7).
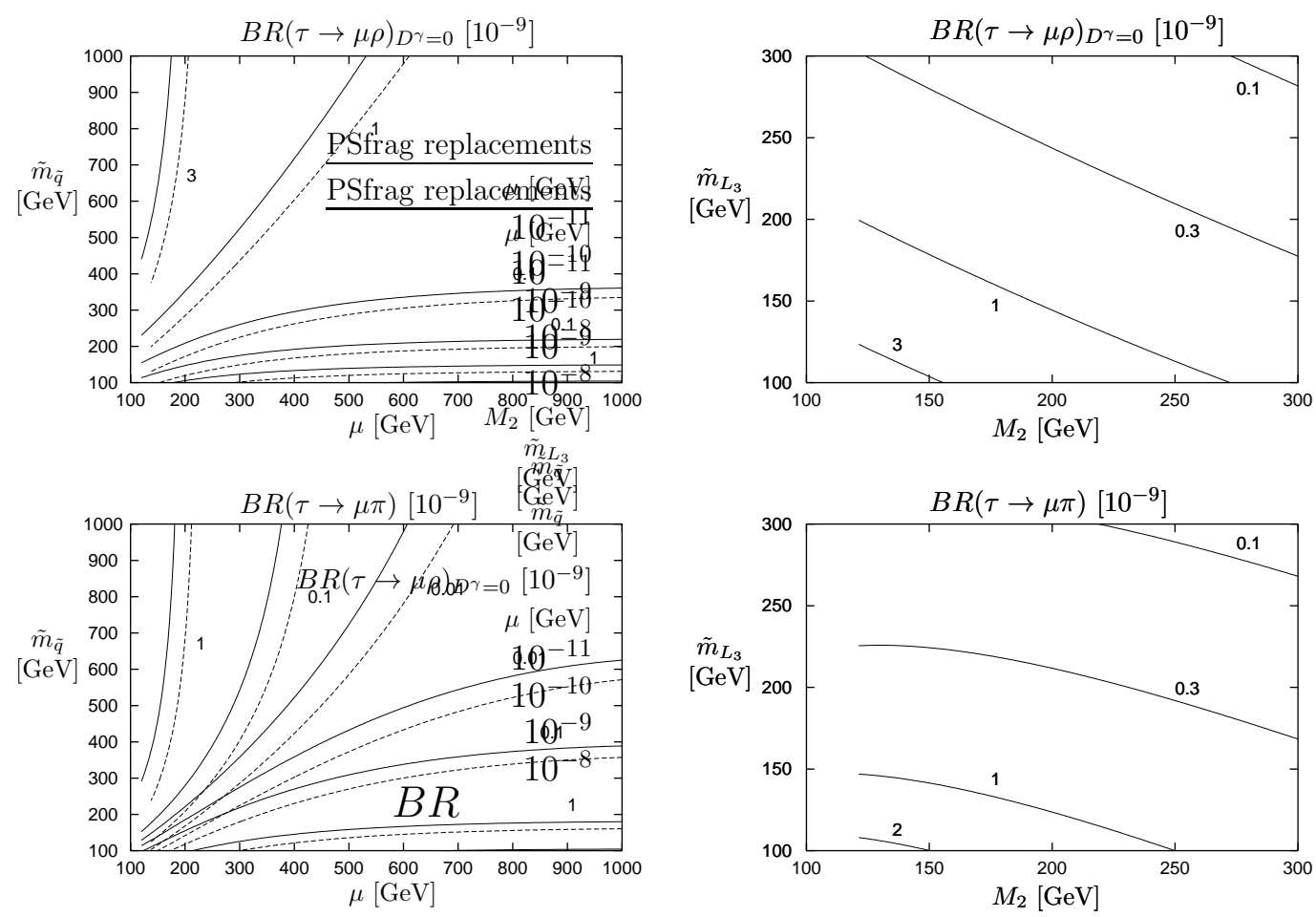

Figure 11: Monopole contribution to $B R(\tau \rightarrow \mu \rho)$ (upper panels) and $B R(\tau \rightarrow \mu \pi)$ (lower panels), for $\tan \beta=3, \tilde{m}_{L_{2}}=1 \mathrm{TeV}, \theta_{L}=\pi / 4$. In the left panels: $\tilde{m}_{L_{3}}=100 \mathrm{GeV}, \tilde{m}_{\tilde{\tau}_{R}}=100 \mathrm{GeV}, M_{2}=120 \mathrm{GeV}$, $M_{1}=100(-100) \mathrm{GeV}$ for the solid (dashed) lines. In the right panels: $\mu=120 \mathrm{GeV}, M_{1}=100 \mathrm{GeV}$, $\tilde{m}_{\tilde{q}}=1 \mathrm{TeV}$ and $\tilde{m}_{\tilde{\tau}_{R}}=100 \mathrm{GeV}$.

In Fig. 11 we depict the monopole contribution to $B R(\tau \rightarrow \mu \rho)$ (upper panels) and $B R(\tau \rightarrow$ $\mu \pi)$ (lower panels), either in the $\left(\mu, \tilde{m}_{\tilde{q}}\right)$ plane (left panels) or in the $\left(M_{2}, \tilde{m}_{L_{3}}\right)$ plane (right panels). Also for these processes cancellation effects are visible (see left panels). Indeed, box contributions interfere destructively with $A_{L}^{Z}$ and $C_{L}^{\gamma}$. The cancellation regions for $\tau \rightarrow \mu \rho$ and 
$\tau \rightarrow \mu \pi$ are somewhat different, also because $C_{L}^{\gamma}$ only contributes to $\tau \rightarrow \mu \rho$. Outside the cancellation regions the monopole-induced $B R(\tau \rightarrow \mu \rho)$ and $B R(\tau \rightarrow \mu \pi)$ can exceed $10^{-9}$. We recall that $\tau \rightarrow \mu \rho$, at variance with $\tau \rightarrow \mu \pi$, also gets a dipole contribution. Notice that eq. (26) can be written as $B R(\tau \rightarrow \mu \rho) \simeq B R_{(D=0)}^{F}+B R_{(F=0)}^{D} \pm 1.5 \sqrt{B R_{(D=0)}^{F} B R_{(F=0)}^{D}}$, where $B R_{(D=0)}^{F}\left(B R_{(F=0)}^{D}\right)$ denotes the pure monopole (dipole) contribution. For instance, if we combine a value $B R_{(D=0)}^{F} \sim 3 \times 10^{-9}$ with the maximal allowed $B R_{(F=0)}^{D} \sim 0.8 \times 10^{-9}$ [eq. (39)], we obtain $B R(\tau \rightarrow \mu \rho) \sim 6 \times 10^{-9}$ or $1.5 \times 10^{-9}$, depending on the interference sign (see also Fig. 7). We have not shown the corresponding examples for $B R(\tau \rightarrow \mu \eta)$ and $B R\left(\tau \rightarrow \mu \eta^{\prime}\right)$, since they exhibit a similar pattern to that of $B R(\tau \rightarrow \mu \pi)$, though the maximal achievable values are somewhat smaller.

\section{2 $(\mathrm{LFV})_{L}$ with large $\tan \beta$ and large masses}

Now we discuss the scenario with large $\tan \beta$. In this case we have a strong enhancement of the $D_{L}^{\gamma(b, c)}$ dipole coefficients, and Higgs- $\mu-\tau$ effective operators become relevant. Two strategies can be envisaged to deal with the former issue. In principle, the parameters can be chosen in such a way that cancellations occur (as illustrated in the previous section) and keep the total $D_{L}^{\gamma}$ dipole coefficient below the bound. However, a significant fine tuning is required, especially if $M_{2}$ and $\tilde{m}_{L_{3}}$ are light. Alternatively, the individual dipole contributions can be kept below the bound by taking large mass parameters. This is the option we pursue here. In this case, the monopole contributions are suppressed, while the Higgs ones are not (for small $m_{A}$ ), because the $\Delta$ coefficients are insensitive to the overall mass scale. Hence, at large $\tan \beta$ and large masses (except for $m_{A}$ ) the following picture emerges.

i) $Z \rightarrow \mu \tau$ is strongly suppressed (even including $D^{Z}$ contributions).

ii) The decays $\tau \rightarrow \mu e e$ and $\tau \rightarrow \mu \rho$ are generically dipole dominated, so they are correlated to $\tau \rightarrow \mu \gamma$ through (34), (36) [and the bounds (37), (39) hold].

iii) The dominant contributions to $B R(\tau \rightarrow \mu \mu \mu)$ [eq. (23)] are induced by the dipole term $D_{L}^{\gamma}$ and the Higgs-mediated term $\delta F_{R}^{\mu_{L}}$, proportional to $\Delta_{L}$ [eq. (59)]. Therefore, $\tau \rightarrow \mu \mu \mu$ is correlated to both $\tau \rightarrow \mu \gamma$ and $A \rightarrow \mu \tau[11]$.

iv) The decay $\tau \rightarrow \mu \eta$ is dominated by the Higgs-mediated terms $\delta F_{L}^{\eta, 8}, \delta F_{L}^{\eta, 0}$ [eqs. (67), (68)]. If $\tau \rightarrow \mu \mu \mu$ is mostly induced by Higgs-exchange, then these processes are correlated as in eq. (69). Higgs-exchange is also relevant for the decays $\tau \rightarrow \mu \pi$ and $\tau \rightarrow \mu \eta^{\prime}$ through the contribution $\delta F_{L}^{\pi}$ [eq. (66)] and $\delta F_{L}^{\eta^{\prime}, 8}, \delta F_{L}^{\eta^{\prime}, 0}$ [analogous to eqs. (67), (68)], respectively.

Let us focus on the processes $\tau \rightarrow \mu \gamma, \tau \rightarrow 3 \mu, A \rightarrow \mu \tau$. The behaviour of these decays is illustrated in Fig. 12 in the plane $\left(|\mu|,\left|M_{2}\right|\right)$ for $\tan \beta=40$. Two examples are shown, where $\operatorname{sign}\left(M_{1} M_{2}\right)$ is either positive (upper panel) or negative (lower panel). The relative sign between $\mu$ and $M_{2}$ is immaterial for large $\tan \beta$, while that between $M_{1}$ and $M_{2}$ matters because it determines the interference between $D_{L}^{\gamma(b)}$ and $D_{L}^{\gamma(c)}$, as well as that between $\Delta_{L}^{(b)}$ and $\Delta_{L}^{(c)}$. In the upper panel $\left[\operatorname{sign}\left(M_{1} M_{2}\right)>0\right]$ the interference is constructive for the $D_{L}^{\gamma}$ components and destructive for the $\Delta_{L}$ ones. Indeed, we can see a cancellation region for $\tau \rightarrow 3 \mu$ and 

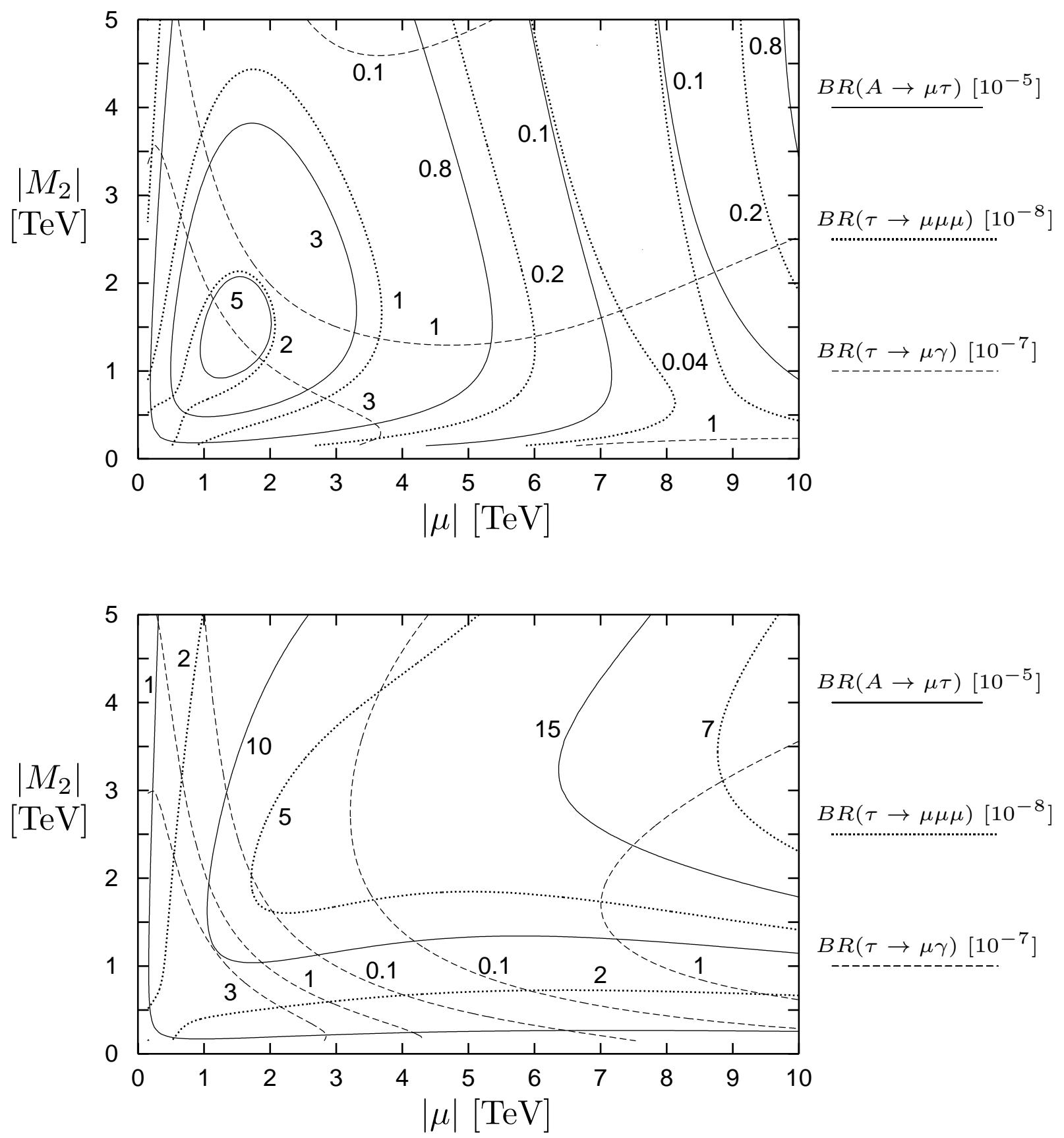

Figure 12: Branching ratios of $\tau \rightarrow \mu \gamma$ (dashed lines), $\tau \rightarrow \mu \mu \mu$ (dotted lines) and $A \rightarrow \mu \tau$ (solid lines) for $\tan \beta=40, \theta_{L}=\pi / 4, \tilde{m}_{L_{2}}=3 \mathrm{TeV}, \tilde{m}_{L_{3}}=1 \mathrm{TeV},\left|M_{1}\right|=\frac{1}{2}\left|M_{2}\right|$. In the upper (lower) panel $\operatorname{sign}\left(M_{1} M_{2}\right)$ is positive (negative) and $\tilde{m}_{\tilde{\tau}_{R}}=\tilde{m}_{\tilde{\mu}_{R}}=2.5 \mathrm{TeV}(2 \mathrm{TeV})$. We have also fixed $m_{A}=100 \mathrm{GeV}$ (which affects $\tau \rightarrow 3 \mu$ ) and $B R(A \rightarrow \tau \tau)=0.1$ (which affects $A \rightarrow \mu \tau$ ). 
$A \rightarrow \mu \tau$ [11]. In the lower panel $\left[\operatorname{sign}\left(M_{1} M_{2}\right)<0\right]$ the opposite situation occurs and we can notice a cancellation region for $\tau \rightarrow \mu \gamma$. In both examples, the contours of $\tau \rightarrow 3 \mu$ and $A \rightarrow \mu \tau$ follow a very similar pattern and are correlated according to eq. (60). This occurs in most of the parameter space shown in Fig. 12 where $\tau \rightarrow 3 \mu$ is indeed essentially dominated by the $\Delta_{L}$ contribution. Deviations occur in the regions where the $D_{L}^{\gamma}$ contribution to $\tau \rightarrow 3 \mu$ is comparable to the $\Delta_{L}$ one (or larger). Regarding the numerical values, in the first example $B R(A \rightarrow \mu \tau)$ and $B R(\tau \rightarrow 3 \mu)$ can reach $5 \times 10^{-5}$ and $2 \times 10^{-8}$, respectively. In the second example $B R(A \rightarrow \mu \tau)$ and $B R(\tau \rightarrow 3 \mu)$ can be larger than $10^{-4}$ and $5 \times 10^{-8}$, respectively. Notice that in Fig. 12 we have fixed $m_{A}=100 \mathrm{GeV}$. Since the Higgs-mediated contribution to $B R(\tau \rightarrow 3 \mu)$ scales as $1 / m_{A}^{4}$, the values in both examples would be accordingly reduced for larger $m_{A}$. Finally, we recall that $B R(\tau \rightarrow \mu \eta)$, which can be inferred by eq. (69), is generically larger ${ }^{14}$ than $B R(\tau \rightarrow 3 \mu)$ and can approach the experimental bound (6). In this case $B R(\tau \rightarrow \mu \pi), B R\left(\tau \rightarrow \mu \eta^{\prime}\right)$ can reach $10^{-9}$ [eqs. (71), (70)].

\section{$5 \quad$ Large $(\mathrm{LFV})_{R}:$ numerical analysis}

In this section we perform a numerical analysis in the case of large (LFV) ${ }_{R}$, assuming vanishing $(\mathrm{LFV})_{L}$, i.e. $\tilde{m}_{L \mu \tau}^{2}=A_{\mu \tau}^{L}=0$. All operator coefficients depend on $\tilde{m}_{R_{\alpha}}, \theta_{R}$ and the bino mass $M_{1}$. Some coefficients also depend on additional parameters. In particular, $A_{R}^{Z(a)}$ and $D_{R}^{\gamma(b)}$ depend on $\mu$ and $\beta ; D_{R}^{\gamma(c)}$ depends on $\mu, \beta, \tilde{m}_{\tilde{\tau}_{L}}, A_{\tau}, A_{\mu \tau}^{R} ; B_{R}^{f}$ depends on $\tilde{m}_{\tilde{f}}$.

The lightest eigenvalue of $\tilde{\mathcal{M}}_{R}^{2}$ is conventionally chosen to be $\tilde{m}_{R_{3}}^{2}$ (although our formulae in Appendix do not depend on such a choice). To enhance (LFV) $)_{R}$, in all our numerical examples we will take maximal mixing, $\theta_{R}=\pi / 4$ (i.e. $\tilde{m}_{R \mu \mu}^{2}=\tilde{m}_{R \tau \tau}^{2}$ in $\tilde{\mathcal{M}}_{R}^{2}$ ), and widely split eigenvalues. Again the mass parameters will be varied in such a way that charged sparticle masses be $\gtrsim 100 \mathrm{GeV}$. The parameter $M_{1}$ will be allowed to reach smaller values without being in conflict with LEP bounds on neutralino production $\left(e^{+} e^{-} \rightarrow \chi_{1}^{0} \chi_{2(3)}^{0}\right)$ and on nonstandard contributions to the invisible $Z$ width $\left(Z \rightarrow \chi_{1}^{0} \chi_{1}^{0}\right)$. We also recall that in the $(\mathrm{LFV})_{R}$ case the parameter $M_{2}$ does not play a direct role and that we do not impose a specific relation between $M_{1}$ and $M_{2}$, hence small $M_{1}$ and large $M_{2}$ can coexist. It is worthwhile to explore low $M_{1}$ values because the monopole coefficients are enhanced. For simplicity, as assumed in Section 4 , we take a common mass $\tilde{m}_{\tilde{e}}$ for selectrons and a common mass $\tilde{m}_{\tilde{q}}$ for first and second generation squarks.

\section{$5.1 \quad(\text { LFV })_{R}$ with small $\tan \beta$}

In analogy with the $(\mathrm{LFV})_{L}$ case, first we focus on the dipole operators and consider a scenario with small $\tan \beta$. The present bound (1) on $\tau \rightarrow \mu \gamma$ translates into $\left|D_{R}^{\gamma}\right| \lesssim 5 \times 10^{-9} \mathrm{GeV}^{-2}$. In Fig. 13 we show contours of $D_{R}^{\gamma(a)}$ (left panel) and $D_{R}^{\gamma(b)}$ (right panel) in the plane $\left(M_{1}, \tilde{m}_{R_{3}}\right)$ with $\theta_{R}=\pi / 4$ and $\tilde{m}_{R_{2}}=1 \mathrm{TeV}$. The $D_{R}^{\gamma(b)}$ plot refers to the choice $\tan \beta=3$ and $\mu=150 \mathrm{GeV}$. We

\footnotetext{
${ }^{14}$ As already emphasized, the value of the ratio (69) is quite sensitive to the parameters $\xi_{s}, \xi_{b}$, which depend on $\mu$, the gluino mass $M_{3}$ and third generation squark mass parameters. Incidentally, $\xi_{b}$ also affects the total
} 

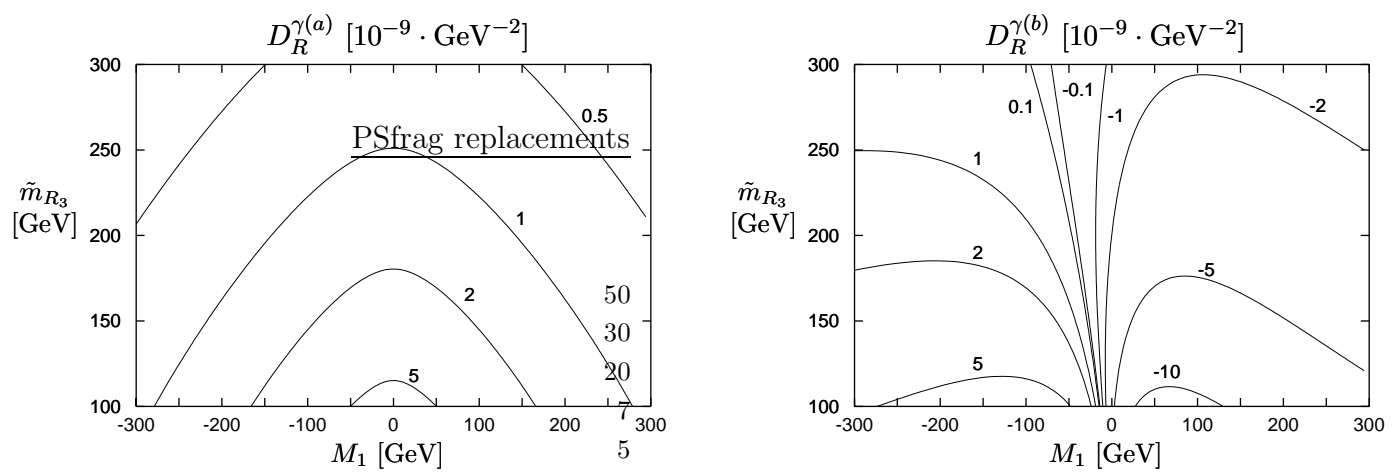

Figure 13: Contours of $D_{R}^{\gamma(a)}$ (left panel) and $D_{R}^{\gamma(b)}$ (right panel) for $\tilde{m}_{R_{2}}=1 \mathrm{TeV}$ and $\theta_{R}=\pi / 4$. To determine $D_{R}^{\gamma(b)}$ we have also fixed $\tan \beta=3$ and $\mu=150 \mathrm{GeV}$.

can see that $D_{R}^{\gamma(a)}$ and $D_{R}^{\gamma(b)}$ are comparable to or even larger than the bound in some regions of the parameter space, and can interfere with each other destructively or constructively. Also $D_{R}^{\gamma(c)}$ may or may not exceed the bound, depending on the range of the extra parameters $\tilde{m}_{\tilde{\tau}_{L}}$, $A_{\tau}, A_{\mu \tau}^{R}$. Anyway, mutual cancellations involving all three contributions can bring the total dipole coefficient $D_{R}^{\gamma}$ below the bound. This is illustrated in Fig. 14, where we plot contours

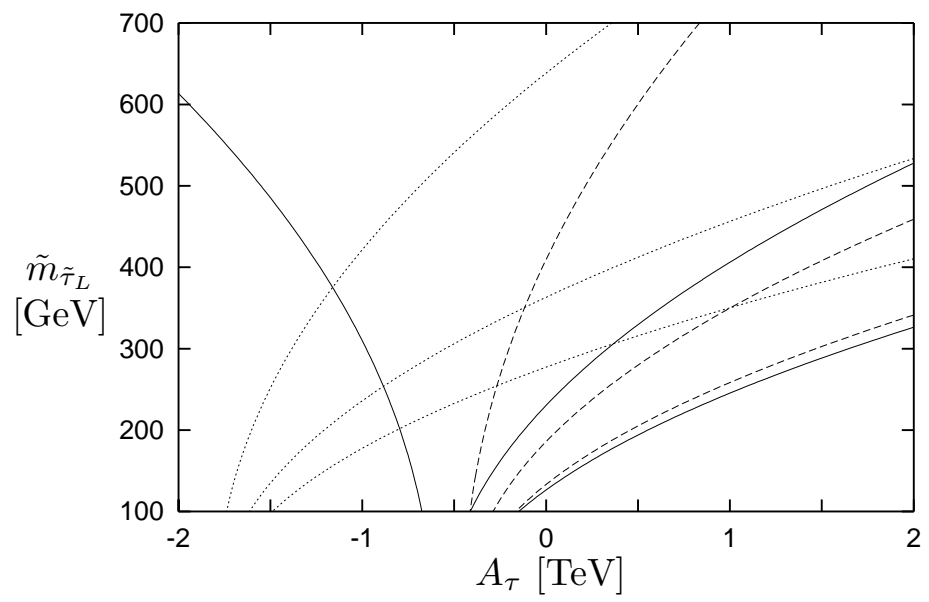

Figure 14: $D_{R}^{\gamma}$ contours for $\tan \beta=3, \theta_{R}=\pi / 4, \tilde{m}_{R_{2}}=1 \mathrm{TeV}, A_{\mu \tau}^{R}=0$ and three choices of $\left(\tilde{m}_{R_{3}}, \mu, M_{1}\right)$ in $\mathrm{GeV}:(100,150,50)$ (dashed), $(150,200,100)$ (solid), (100,600,-50) (dotted). For each example the two external lines correspond to $\left|D_{R}^{\gamma}\right|=5 \times 10^{-9} \mathrm{GeV}^{-2}$ and the middle one to $D_{R}^{\gamma}=0$.

of $D_{R}^{\gamma}\left( \pm 5 \times 10^{-9} \mathrm{GeV}^{-2}, 0\right)$ in the plane $\left(A_{\tau}, \tilde{m}_{\tilde{\tau}_{L}}\right)$ for $\tan \beta=3, \tilde{m}_{R_{2}}=1 \mathrm{TeV}, A_{\mu \tau}^{R}=0$ and three choices of $\left(\tilde{m}_{R_{3}}, \mu, M_{1}\right)$. As a general feature the allowed regions are wider than in the $(\mathrm{LFV})_{L}$ examples (see Fig. 6) because the individual dipole contributions are now smaller. In particular, it is easier to fulfil the bound even with vanishing trilinear terms.

In Fig. 15 we show the branching ratios of the different LFV processes in two examples, where $\mu$ is either small (upper panel) or large (lower panel). In each case the dipole $D_{R}^{\gamma}$ $\overline{A \text { width (through the main channel } A \rightarrow b \bar{b})}$ and hence $B R(A \rightarrow \tau \tau)$ and $B R(A \rightarrow \mu \tau)$. 

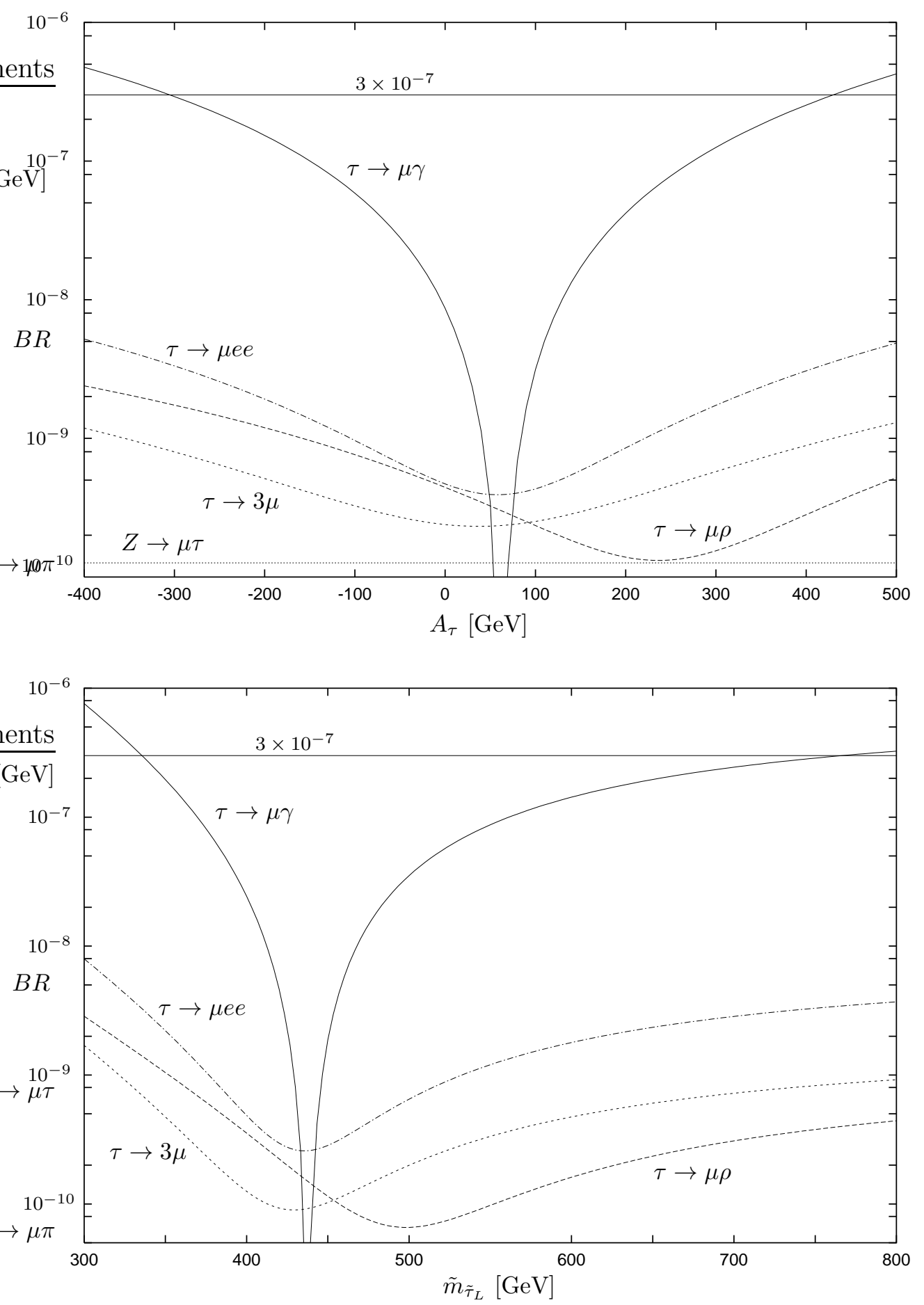

Figure 15: Branching ratios of LFV decays for $\tan \beta=3, \tilde{m}_{R_{2}}=1 \mathrm{TeV}, \tilde{m}_{R_{3}}=100 \mathrm{GeV}, \theta_{R}=\pi / 4$, $A_{\mu \tau}^{R}=0$ and $\tilde{m}_{\tilde{e}}=100 \mathrm{GeV}, \tilde{m}_{\tilde{q}}=200 \mathrm{GeV}$. In the upper panel, the remaining parameters are: $\mu=150 \mathrm{GeV}$, $M_{1}=50 \mathrm{GeV}, \tilde{m}_{\tilde{\tau}_{L}}=\tilde{m}_{\tilde{\mu}_{L}}=200 \mathrm{GeV}$. In the lower panel: $\mu=800 \mathrm{GeV}, M_{1}=-50 \mathrm{GeV}, A_{\tau}=0$ and $\tilde{m}_{\tilde{\mu}_{L}}=\tilde{m}_{\tilde{\tau}_{L}}$. The solid horizontal line indicates the present bound on $B R(\tau \rightarrow \mu \gamma)$. In the upper example, $B R(\tau \rightarrow \mu \pi) \sim 2 \times 10^{-11}, B R(\tau \rightarrow \mu \eta) \sim 2 \times 10^{-12}, B R\left(\tau \rightarrow \mu \eta^{\prime}\right) \sim 4 \times 10^{-11}$ and $B R(Z \rightarrow \mu \tau) \sim 10^{-10}$. In the lower example, $B R(\tau \rightarrow \mu \pi) \sim 3 \times 10^{-12}, B R(\tau \rightarrow \mu \eta) \sim 4 \times 10^{-12}, B R\left(\tau \rightarrow \mu \eta^{\prime}\right) \sim 10^{-11}$ and $B R(Z \rightarrow \mu \tau) \sim 2 \times 10^{-11}$. 

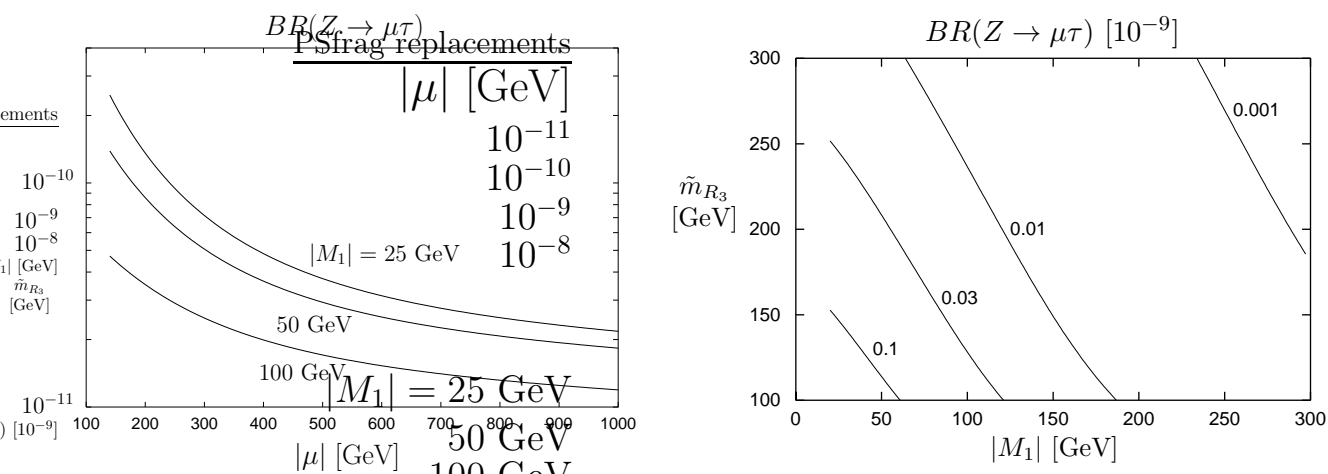

Figure 16: $B R(Z \rightarrow \mu \tau)$ for $\tan \beta=3, \tilde{m}_{R_{2}}=1 \mathrm{TeV}, \theta_{R}=\pi / 4$ and $\tilde{m}_{\tilde{\tau}_{L}}=300 \mathrm{GeV}$. In the left panel: $\tilde{m}_{R_{3}}=100 \mathrm{GeV}$ and $\left|M_{1}\right|$ as shown. In the right panel: $|\mu|=150 \mathrm{GeV}$.

contribution is varied (through either $A_{\tau}$ or $\tilde{m}_{\tilde{\tau}_{L}}$ ) and the monopole ones are essentially fixed. The behaviour of $B R(\tau \rightarrow \mu \mu \mu), B R(\tau \rightarrow \mu e e), B R(\tau \rightarrow \mu \rho)$ reflects the interplay between the dipole and monopole contributions, similarly to the $(\mathrm{LFV})_{L}$ case. Now let us examine in more detail the parameter dependence of monopole contributions, considering one process at a time.

In Fig. $16 B R(Z \rightarrow \mu \tau)$ is plotted as a function of $|\mu|$ (left panel) and in the $\left(\left|M_{1}\right|, \tilde{m}_{R_{3}}\right)$ plane for small $\mu$ (right panel). At variance with the (LFV $)_{L}$ case, the interference between the $A^{Z}$ and $C^{Z}$ contributions is now constructive. For small $\mu$ we have $\left|A_{R}^{Z}\right| \gtrsim\left|C_{R}^{Z}\right|$. For large $\mu,\left|A_{R}^{Z}\right|$ is suppressed. [The $A_{R}^{Z(c)}$ component grows with $\mu$, but it remains small $\operatorname{since} \tan \beta$ is small. A similar comment applies to $\left|D_{R}^{Z(c)}\right|$.] Notice that $B R(Z \rightarrow \mu \tau)$ does not reach $\sim 10^{-9}$, even for small mass parameters. This is in contrast with the $(\mathrm{LFV})_{L}$ case.

In Fig. 17 the monopole contribution to $B R(\tau \rightarrow \mu \mu \mu)$ is shown as a function of $|\mu|$ (left panel) and in the $\left(\left|M_{1}\right|, \tilde{m}_{R_{3}}\right)$ plane (right panel). For $\left|M_{1}\right| \gtrsim 50 \mathrm{GeV}$ we have $B R(\tau \rightarrow$ $\mu \mu \mu)_{D^{\gamma=0}} \lesssim 10^{-10}$, while values close to $10^{-9}$ can only be achieved for quite a small $\left|M_{1}\right|$. We recall the pure dipole contribution to $B R(\tau \rightarrow \mu \mu \mu)$ cannot exceed $10^{-9}$ [eq. (38)].

In Fig. 18 the monopole contribution to $B R(\tau \rightarrow \mu e e)$ is plotted in the $\left(|\mu|, \tilde{m}_{\tilde{e}}\right)$ plane (left panel) and in the $\left(\left|M_{1}\right|, \tilde{m}_{R_{3}}\right)$ plane (right panel). Values $\mathcal{O}\left(10^{-9}\right)$ can only be reached for small $\left|M_{1}\right|$. The overall $B R(\tau \rightarrow \mu e e)$ can be few times $10^{-9}$ when the maximal allowed dipole contribution is accounted (see also Fig. 15).

In Fig. 19 we show the monopole contribution to $B R(\tau \rightarrow \mu \rho)$ (solid) and $B R(\tau \rightarrow \mu \pi)$ (dashed), either in the $\left(|\mu|, \tilde{m}_{\tilde{q}}\right)$ plane (left panel) or in the $\left(\left|M_{1}\right|, \tilde{m}_{R_{3}}\right)$ plane (right panel). In the left panel cancellation effects are visible in $B R(\tau \rightarrow \mu \pi)$, and are due to a destructive interference between $A_{R}^{Z}$ and box contributions. Even outside such an interference region, however, the $\tau \rightarrow \mu \pi$ decay is strongly disfavoured, as its $B R$ can hardly reach $10^{-10}$. Regarding $\tau \rightarrow \mu \rho$, we see that the pure monopole contribution can exceed $10^{-10}$ for $\left|M_{1}\right| \lesssim 100 \mathrm{GeV}$. Suppose the latter contribution is $0.3 \times 10^{-9}$ and the pure dipole one is $0.8 \times 10^{-9}$ [maximal value, see eq. (39)]. Then we have $B R(\tau \rightarrow \mu \rho) \sim 2 \times 10^{-9}$ or $0.4 \times 10^{-9}$, depending on the interference sign (see also Fig. 15). As regards $\tau \rightarrow \mu \eta$, the $B R$ exhibits a pattern similar to that of $\tau \rightarrow \mu \pi$, but its maximal values are even smaller. In the case of $\tau \rightarrow \mu \eta^{\prime}$, the interference 

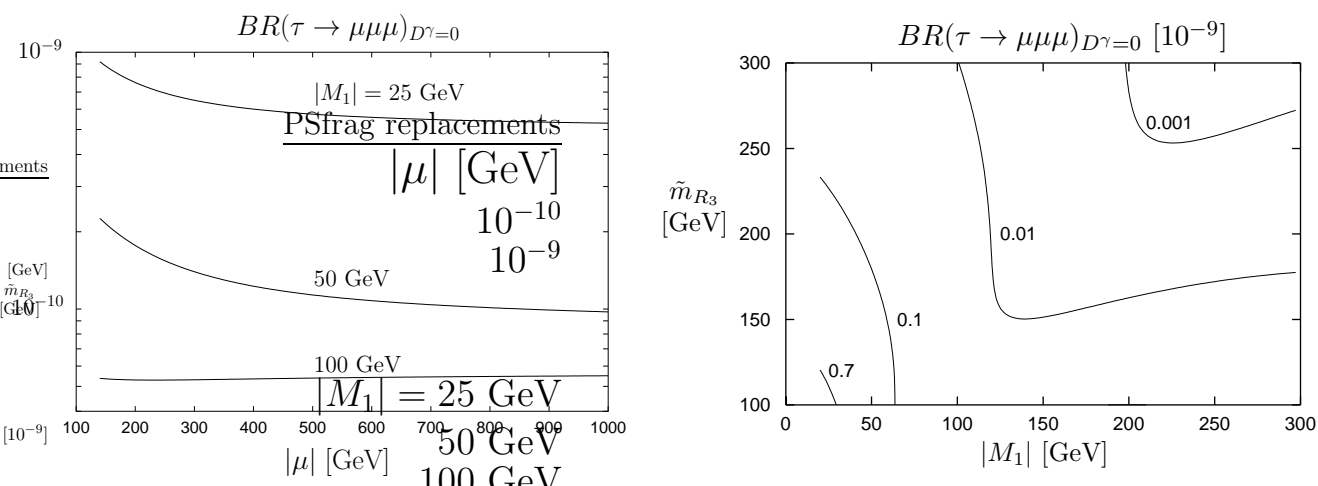

Figure 17: Monopole contribution to $B R(\tau \rightarrow \mu \mu \mu)$ for $\tan \beta=3, \tilde{m}_{R_{2}}=1 \mathrm{TeV}, \theta_{R}=\pi / 4$ and $\tilde{m}_{\tilde{\tau}_{L}}=$ $\tilde{m}_{\tilde{\mu}_{L}}=300 \mathrm{GeV}$. In the left panel: $\tilde{m}_{R_{3}}=100 \mathrm{GeV}$ and $\left|M_{1}\right|$ as shown. In the right panel: $|\mu|=150 \mathrm{GeV}$.
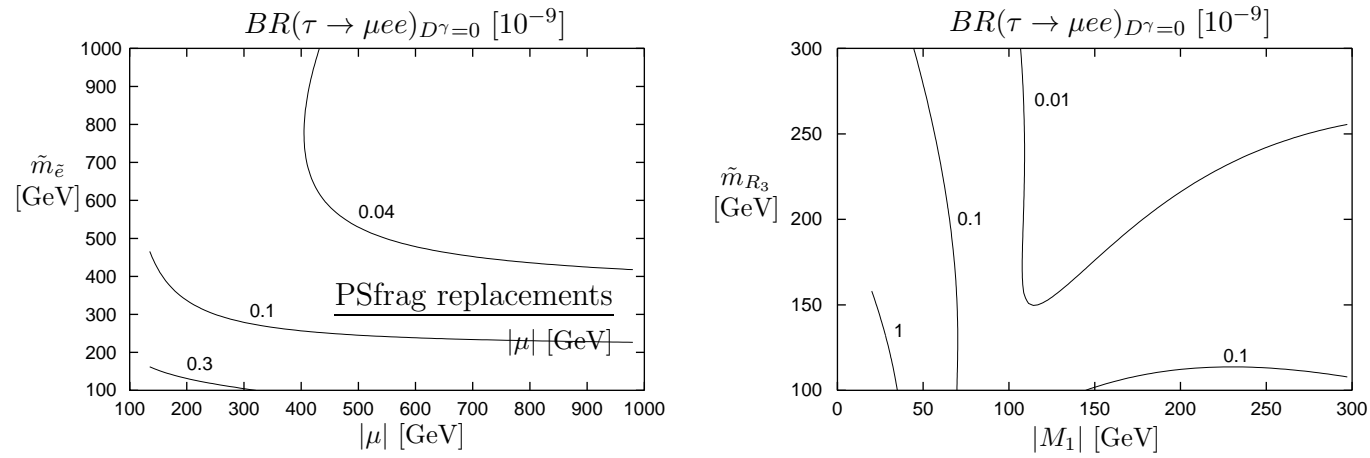

Figure 18: Monopole contribution to $B R(\tau \rightarrow \mu e e)$ for $\tan \beta=3, \tilde{m}_{R_{2}}=1 \mathrm{TeV}, \theta_{R}=\pi / 4$ and $\tilde{m}_{\tilde{\tau}_{L}}=$ $300 \mathrm{GeV}$. In the left panel: $\tilde{m}_{R_{3}}=100 \mathrm{GeV},\left|M_{1}\right|=50 \mathrm{GeV}$. In the right panel: $|\mu|=150 \mathrm{GeV}, \tilde{m}_{\tilde{e}}=100 \mathrm{GeV}$.
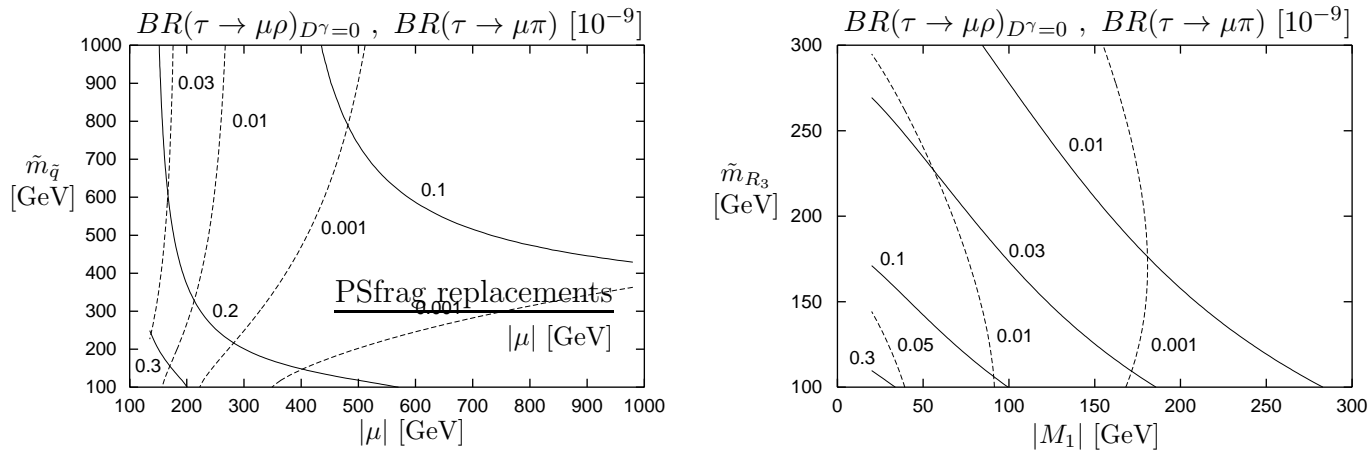

Figure 19: Monopole contribution to $B R(\tau \rightarrow \mu \rho)$ (solid lines) and $B R(\tau \rightarrow \mu \pi)$ (dashed lines), for $\tan \beta=3$, $\tilde{m}_{R_{2}}=1 \mathrm{TeV}, \theta_{R}=\pi / 4$ and $\tilde{m}_{\tilde{\tau}_{L}}=300 \mathrm{GeV}$. In the left panel: $\tilde{m}_{R_{3}}=100 \mathrm{GeV},\left|M_{1}\right|=50 \mathrm{GeV}$. In the right panel: $|\mu|=150 \mathrm{GeV}, \tilde{m}_{\tilde{q}}=500 \mathrm{GeV}$. 
between $A_{R}^{Z}$ and box contributions tends to be constructive, and the $B R$ could reach $10^{-10}$.

\section{$5.2 \quad(\text { LFV })_{R}$ with large $\tan \beta$ and large masses}

To discuss large $(\mathrm{LFV})_{R}$ in the large $\tan \beta$ limit we follow an approach similar to that adopted in Section 4.2. In this limit the Higgs- $\mu-\tau$ effective operators can play an important role. The enhanced contributions to the $D_{R}^{\gamma(b, c)}$ dipole coefficients can be kept under control either through mutual cancellations (for small mass parameters) or by taking large masses. The former option requires some fine tuning, although this is milder than in the $(\mathrm{LFV})_{L}$ case. Among monopole coefficients, $A_{R}^{Z}$ can be slightly larger for large $\tan \beta\left[A_{R}^{Z(a)}\right.$ is proportional to $\cos 2 \beta$, which gets closer to -1 , and $A_{R}^{Z(b, c)}$ can play some role]. Let us consider in more detail the second possibility, i.e. the large mass limit (with small $m_{A}$ ). In this case all monopole contributions are suppressed

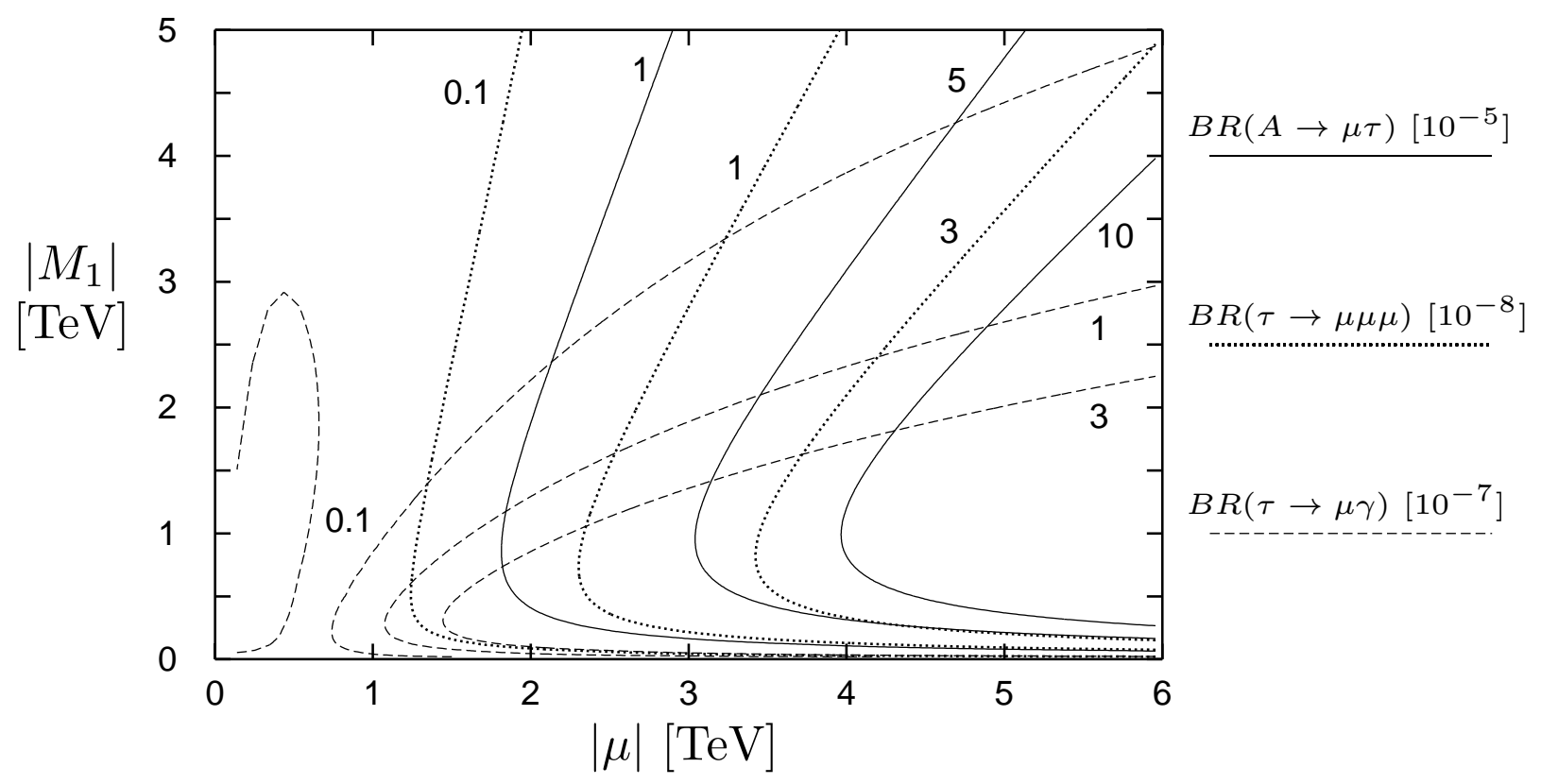

Figure 20: Branching ratios of $\tau \rightarrow \mu \gamma$ (dashed lines), $\tau \rightarrow \mu \mu \mu$ (dotted lines) and $A \rightarrow \mu \tau$ (solid lines) for $\tan \beta=40, \theta_{R}=\pi / 4, \tilde{m}_{R_{2}}=3 \mathrm{TeV}, \tilde{m}_{R_{3}}=0.7 \mathrm{TeV}$ and $\tilde{m}_{\tilde{\tau}_{L}}=0.7 \mathrm{TeV}$. We have also fixed $m_{A}=100 \mathrm{GeV}$ and $B R(A \rightarrow \tau \tau)=0.1$.

and so is $Z \rightarrow \mu \tau$. The decays $\tau \rightarrow \mu e e$ and $\tau \rightarrow \mu \rho$ are generically dipole dominated, so the relations (34), (36) [and the bounds (37), (39)] hold. The dominant contributions to $B R(\tau \rightarrow \mu \mu \mu)$ [eq. (23)] are induced by the dipole term $D_{R}^{\gamma}$ and the Higgs-mediated term $\delta F_{L}^{\mu_{R}}$, proportional to $\Delta_{R}$ [eq. (59)]. Therefore, $\tau \rightarrow \mu \mu \mu$ is correlated to both $\tau \rightarrow \mu \gamma$ and $A \rightarrow \mu \tau$ [11]. The processes $\tau \rightarrow \mu \eta, \tau \rightarrow \mu \eta^{\prime}$ and $\tau \rightarrow \mu \pi$ are essentially induced by the Higgsexchange terms $\delta F_{R}^{\eta, 8}, \delta F_{R}^{\eta, 0}, \delta F_{R}^{\eta^{\prime}, 8}, \delta F_{R}^{\eta^{\prime}, 0}$ and $\delta F_{R}^{\pi}$, so they are correlated to one another [eqs. (70), (71)], to $\tau \rightarrow 3 \mu$ [eq. (69)], and to $A \rightarrow \mu \tau$. 
In Fig. 20 we show the $B R \mathrm{~s}$ of $\tau \rightarrow \mu \gamma, \tau \rightarrow 3 \mu, A \rightarrow \mu \tau$ in the plane $\left(|\mu|,\left|M_{1}\right|\right)$ for $\tan \beta=40$. In the left side of the plot all decays are suppressed, in part because of internal cancellations both in $D_{R}^{\gamma}$ (visible in the figure) and in $\Delta_{R}$ (not shown here, see Fig. 3 in [11]). All $B R$ s increase for increasing $\mu$ because $D_{R}^{\gamma(c)}$ and $\Delta_{R}^{(c)}$ dominate. The pattern of the contours for $\tau \rightarrow \mu \gamma$ is different from that of $\tau \rightarrow 3 \mu$ and $A \rightarrow \mu \tau$, whose rates are correlated to each other in the regions where $\tau \rightarrow 3 \mu$ is dominated by the $\Delta_{R}$ contribution [see eq. (60)]. In particular, there is a region where $B R(\tau \rightarrow \mu \gamma)$ is below the present bound (1), whereas $B R(A \rightarrow \mu \tau)$ and $B R(\tau \rightarrow 3 \mu)$ can reach $10^{-4}$ and $5 \times 10^{-8}$, respectively. Also notice that we have fixed $m_{A}=100 \mathrm{GeV}: B R(\tau \rightarrow 3 \mu)$ is smaller for larger $m_{A}$, since the Higgs-mediated contribution scales as $1 / m_{A}^{4}$. The largest values of $B R(\tau \rightarrow 3 \mu)$ are correlated to values of $B R(\tau \rightarrow \mu \eta)$ close to its present bound (6), and to values of $B R(\tau \rightarrow \mu \pi)$ and $B R\left(\tau \rightarrow \mu \eta^{\prime}\right)$ of order $10^{-9}$.

\section{Conclusions}

The observation of LFV processes would be a clear evidence of physics beyond the Standard Model. In this paper we have focused our attention on LFV in the $\mu-\tau$ sector by considering several LFV decays, namely $\tau \rightarrow \mu X\left(X=\gamma, e^{+} e^{-}, \mu^{+} \mu^{-}, \rho, \pi, \eta, \eta^{\prime}\right), Z \rightarrow \mu \tau$ and Higgs boson decays into $\mu \tau$. First we have presented a model independent treatment of such decays, then we have analysed them in a general unconstrained MSSM framework, without assuming specific relations among mass parameters. In particular, we have allowed slepton mass matrices to have large $\mu-\tau$ entries (whose origin is unspecified). This possibility is not excluded by the present experimental bounds [essentially that on $B R(\tau \rightarrow \mu \gamma)$ ], even for a relatively light superparticle spectrum. It is well known, instead, that the analogous situation for $e-\mu$ flavour violation is strongly disfavoured by the stringent bound $B R(\mu \rightarrow e \gamma)<1.2 \times 10^{-11}$ [35]. We have implicitly assumed that $\mu-e$ flavour transitions are adequately suppressed by sufficiently small $e-\mu$ and $e-\tau$ entries in the slepton mass matrices. Issues such as the generation and the amount of LFV, as well as other model-dependent aspects, go beyond the scope of our paper and can only be addressed in specific frameworks (see e.g. [36, 18, 19, 37]).

We have analysed the behaviour of the various LFV processes in the MSSM parameter space in terms of the relevant operator coefficients. In particular, we have disentangled the dipole and non-dipole contributions to the LFV decays in order to better appreciate the correlations among them. We have provided general formulae for the operator coefficients in Appendix and presented a numerical analysis with several examples in Sections 4 and 5. For the sake of brevity, we can try to summarize our results by schematically distinguishing a few different scenarios. For definiteness, consider the case of large $(\mathrm{LFV})_{L}$.

1. Each of the contributions to the photon dipole operator fulfils the bound dictated by $\tau \rightarrow \mu \gamma$, so no mutual cancellations among them need to be invoked. Also suppose that MSSM mass parameters have comparable magnitudes, with the possible exception of $m_{A}$. For convenience, we distinguish the cases of small and large $\tan \beta$. i) For small $\tan \beta$, mass parameters should be larger than a few hundreds GeV to accomplish the $\tau \rightarrow \mu \gamma$ bound. This implies that monopole contributions to all branching ratios are generically smaller than $10^{-10}$. Also the Higgs-mediated contributions are small, because $\tan \beta$ is small. 
Hence $\tau \rightarrow \mu P\left(P=\pi, \eta, \eta^{\prime}\right)$ and $Z \rightarrow \mu \tau$ have $B R$ s smaller than $10^{-10}$. The processes $\tau \rightarrow \mu e e, \tau \rightarrow 3 \mu, \tau \rightarrow \mu \rho$ tend to be dipole-dominated [eqs. (34), (35), (36)], and their $B R$ s can be $\mathcal{O}\left(10^{-9}\right)$ if $B R(\tau \rightarrow \mu \gamma)$ is close to its present bound [see eqs. (37), (38), (39)]. ii) For large $\tan \beta$, the mass parameters have to be pushed towards the TeV range to accomplish the $\tau \rightarrow \mu \gamma$ bound. The monopole contributions are even more suppressed than in the previous case. On the other hand, the Higgs-mediated contributions are strongly enhanced and may become comparable to or even more important than the dipole ones (for small $m_{A}$ ). Hence the processes $\tau \rightarrow 3 \mu, \tau \rightarrow \mu \eta$ become excellent signatures, together with LFV Higgs boson decays, to which they are correlated. Indeed, one can have $B R(A \rightarrow \mu \tau) \sim 10^{-4}$ and, for small $m_{A}, B R(\tau \rightarrow 3 \mu)$ close to $10^{-7}$, with $B R(\tau \rightarrow \mu \eta)$ even larger than the latter one [and $B R(\tau \rightarrow \mu \pi), B R\left(\tau \rightarrow \mu \eta^{\prime}\right)$ possibly reaching $\left.10^{-9}\right]$. Regarding the other processes (and also $\tau \rightarrow 3 \mu, \tau \rightarrow \mu P$ for large $m_{A}$ ), the conclusions drawn in case $i$ ) hold again.

2. The dipole operator satisfies the $\tau \rightarrow \mu \gamma$ bound due to cancellations among individual contributions and/or because of hierarchies among mass parameters (e.g. with large $\mu$ ), like in the examples displayed in Figs. 6 and 7. Such cases are interesting because $\tilde{m}_{L_{3}}$ and $M_{2}$ are not forced to be large, so monopole operators could contribute to each branching ratio at the level of $10^{-9}$ (or more), unless cancellations occur among different monopole components (which indeed could happen, as we have shown). Such contributions can bring $B R(\tau \rightarrow \mu P)\left(P=\pi, \eta, \eta^{\prime}\right)$ and $B R(Z \rightarrow \mu \tau)$ to values $\mathcal{O}\left(10^{-9}\right)$, and an interesting interplay between dipole and monopole contributions can take place in the other processes. This breaks the direct correlation [eqs. (34), (35), (36)] between $\tau \rightarrow \mu \gamma$ and $\tau \rightarrow \mu e e, \tau \rightarrow 3 \mu, \tau \rightarrow \mu \rho$. For each of the latter processes, the $B R$ can be smaller or larger than its maximal $D^{\gamma}$-dominated value, and can become a few $\times 10^{-9}$. Such values can be reached even in the case of strongly suppressed dipole contributions. In this extreme case $\tau \rightarrow \mu e e, \tau \rightarrow 3 \mu, \tau \rightarrow \mu \rho$ could be as sensitive as $\tau \rightarrow \mu \gamma$ to supersymmetric LFV effects. All these comments apply to the small $\tan \beta$ case. If we insist on having small $\tilde{m}_{L_{3}}$ and $M_{2}$ with large $\tan \beta$, a strong fine-tuning is required to keep the total $D^{\gamma}$ below the bound (through cancellations). In this special situation, the Higgs-mediated contributions could push $B R(\tau \rightarrow 3 \mu)$ and $B R(\tau \rightarrow \mu \eta)$ to values $\mathcal{O}\left(10^{-7}\right)$ for small $m_{A}$, and $Z$-dipole contributions could help $B R(Z \rightarrow \mu \tau)$ to reach $10^{-9}$.

The case of large $(\mathrm{LFV})_{R}$ can be summarized along similar lines, although some differences with respect to $(\mathrm{LFV})_{L}$ are present (see Section 5 for details). For instance, it is easier to fulfil the $\tau \rightarrow \mu \gamma$ bound on the dipole operator with small mass parameters, especially at small $\tan \beta$. Monopole contributions are typically smaller than in the $(\mathrm{LFV})_{L}$ case, unless $M_{1}$ is stretched to very small values. Barring this possibility, and assuming that $\tau \rightarrow \mu \gamma$ is close to its present bound, the only processes whose $B R$ s can be $\mathcal{O}\left(10^{-9}\right)$ at small $\tan \beta$ are $\tau \rightarrow \mu e e, \tau \rightarrow 3 \mu, \tau \rightarrow \mu \rho$, mainly through dipole contributions. At large tan $\beta$, also Higgsmediated contributions can play an important role. If $\mu$ is larger than other mass parameters and $m_{A}$ is small, $B R(\tau \rightarrow 3 \mu)$ and $B R(\tau \rightarrow \mu \eta)$ can reach values $\mathcal{O}\left(10^{-7}\right)$.

As a final remark, we recall that future collider experiments will give crucial information (or constraints) on the MSSM parameters, including those related to LFV. Indeed, supersymmetric 
$\mu-\tau$ LFV effects can also show up, already at the tree level, in the decays of superparticles directly produced at LHC or leptonic colliders [38]. This important search will be complementary to that of the loop-induced LFV decays discussed in our work.

\section{Acknowledgments}

This work was partially supported by MIUR and by the European Union under the contracts HPRN-CT-2000-00148 (Across the Energy Frontier) and HPRN-CT-2000-00149 (Collider Physics).

This article is dedicated to the memory of Anna's parents.

\section{A Operator coefficients in the MSSM}

\section{A.1 MSSM Lagrangian and conventions}

We recall here the lagrangian terms relevant for our computations, and in this way also establish our conventions and notations. The gauge bosons we need are the neutral ones (photon and $Z)$. The covariant kinetic term for a generic Weyl fermion $\psi$ is $i \bar{\psi} \bar{\sigma}^{\mu} D_{\mu} \psi$, where $\psi$ can be either a matter fermion $\left(f, f^{c}\right)$ or a gaugino $\left(\tilde{B}^{0}, \tilde{W}^{0}, \tilde{W}^{ \pm}\right)$or a higgsino $\left(\tilde{H}_{1}^{0}, \tilde{H}_{2}^{0}, \tilde{H}_{1}^{-}, \tilde{H}_{2}^{+}\right)$, and the covariant derivative reads as:

$$
D_{\mu} \psi=\left(\partial_{\mu}+i e Q_{\psi} A_{\mu}+i g_{Z}\left(T_{\psi}^{3}-Q_{\psi} s_{W}^{2}\right) Z_{\mu}\right) \psi
$$

with $g_{Z}=g / c_{W}\left(c_{W}=\cos \theta_{W}, s_{W}=\sin \theta_{W}\right.$, where $\theta_{W}$ is the weak mixing angle). Electric charge $\left(Q_{\psi}\right)$ is related to weak isospin $\left(T_{\psi}^{3}\right)$ and hypercharge $\left(Y_{\psi}\right)$ through $Q_{\psi}=T_{\psi}^{3}+Y_{\psi}$. Similarly, for a generic complex scalar $\phi\left(\tilde{f}=\tilde{f}_{L}, \tilde{f}^{c}=\tilde{f}_{R}^{*}, H_{1}^{0}, H_{2}^{0}, H_{1}^{-}, H_{2}^{+}\right)$, we have $\left(D^{\mu} \phi\right)^{*}\left(D_{\mu} \phi\right)$. The gaugino and higgsino mass terms are:

$$
-\frac{1}{2} M_{1} \tilde{B}^{0} \tilde{B}^{0}-\frac{1}{2} M_{2} \tilde{W}^{0} \tilde{W}^{0}-M_{2} \tilde{W}^{+} \tilde{W}^{-}-\mu\left(\tilde{H}_{1}^{0} \tilde{H}_{2}^{0}-\tilde{H}_{1}^{-} \tilde{H}_{2}^{+}\right)+\text {h.c.. }
$$

Notice that our sign convention for the $\mu$ parameter is opposite to the one commonly used. The gaugino masses and the $\mu$ parameter will be taken real in the diagrammatic computations. The relevant fermion-sfermion-gaugino interaction terms are:

$$
\begin{aligned}
& -g^{\prime} \sqrt{2}\left(Y_{f} \tilde{f}^{*} f+Y_{f^{c}} \tilde{f}^{c *} f^{c}-\frac{1}{2} H_{1}^{0 *} \tilde{H}_{1}^{0}+\frac{1}{2} H_{2}^{0 *} \tilde{H}_{2}^{0}\right) \tilde{B}^{0} \\
& -g \sqrt{2}\left(T_{f}^{3} \tilde{f}^{*} f+\frac{1}{2} H_{1}^{0 *} \tilde{H}_{1}^{0}-\frac{1}{2} H_{2}^{0 *} \tilde{H}_{2}^{0}\right) \tilde{W}^{0} \\
& -g\left(\tilde{f}_{u}^{*} f_{d}+H_{1}^{0 *} \tilde{H}_{1}^{-}\right) \tilde{W}^{+}-g\left(\tilde{f}_{d}^{*} f_{u}+H_{2}^{0 *} \tilde{H}_{2}^{+}\right) \tilde{W}^{-}+\text {h.c. }
\end{aligned}
$$

where $f_{u, d}\left(\tilde{f}_{u, d}\right)$ are up- or down-type components of fermion (sfermion) doublets. The $h_{\tau^{-}}$ Yukawa interactions we use are:

$$
-h_{\tau} H_{1}^{0} \tau^{c} \tau-h_{\tau} \tilde{\tau}^{c} \tilde{H}_{1}^{0} \tau-h_{\tau} \tau^{c}\left(\tilde{H}_{1}^{0} \tilde{\tau}-\tilde{H}_{1}^{-} \tilde{\nu}_{\tau}\right)+\text { h.c. }
$$


Analogous expressions hold for other Yukawa couplings (in particular, $h_{\mu}$ ).

The (soft-breaking) scalar mass terms have the generic form $-\left.\tilde{m}_{f}^{2}\left|\tilde{f}^{2}-\tilde{m}_{f^{c}}^{2}\right| \tilde{f} c\right|^{2}$ (equivalently denoted as $\left.-\tilde{m}_{\tilde{f}_{L}}^{2}\left|\tilde{f}_{L}\right|^{2}-\tilde{m}_{\tilde{f}_{R}}^{2}\left|\tilde{f}_{R}\right|^{2}\right)$. We assume that such terms are flavour conserving, except for those in the smuon-stau sector, which require a more detailed description. In the left sector the relevant terms are:

$$
-\tilde{L}_{\mu}^{\dagger} \tilde{m}_{L \mu \mu}^{2} \tilde{L}_{\mu}-\tilde{L}_{\tau}^{\dagger} \tilde{m}_{L \tau \tau}^{2} \tilde{L}_{\tau}-\left(\tilde{L}_{\mu}^{\dagger} \tilde{m}_{L \mu \tau}^{2} \tilde{L}_{\tau}+\text { h.c. }\right)
$$

where $\tilde{L}_{\mu}=\left(\tilde{\nu}_{\mu}, \tilde{\mu}_{L}\right)^{T}, \tilde{L}_{\tau}=\left(\tilde{\nu}_{\tau}, \tilde{\tau}_{L}\right)^{T}$. In the right sector the relevant terms are:

$$
-\tilde{\mu}_{R}^{*} \tilde{m}_{R \mu \mu}^{2} \tilde{\mu}_{R}-\tilde{\tau}_{R}^{*} \tilde{m}_{R \tau \tau}^{2} \tilde{\tau}_{R}-\left(\tilde{\mu}_{R}^{*} \tilde{m}_{R \mu \tau}^{2} \tilde{\tau}_{R}+\text { h.c. }\right) .
$$

Finally, in the left-right sector the terms with neutral Higgs bosons are:

$$
-h_{\mu}\left(\mu H_{2}^{0 *}+A_{\mu} H_{1}^{0}\right) \tilde{\mu}_{R}^{*} \tilde{\mu}_{L}-h_{\tau}\left(\mu H_{2}^{0 *}+A_{\tau} H_{1}^{0}\right) \tilde{\tau}_{R}^{*} \tilde{\tau}_{L}-h_{\tau} A_{\mu \tau}^{L} H_{1}^{0} \tilde{\tau}_{R}^{*} \tilde{\mu}_{L}-h_{\tau} A_{\mu \tau}^{R} H_{1}^{0} \tilde{\mu}_{R}^{*} \tilde{\tau}_{L}+\text { h.c. }
$$

where $A_{\mu}, A_{\tau}, A_{\mu \tau}^{L, R}$ are mass parameters, which will be taken real in the diagrammatic computations.

The mass parameters in eqs. (78-79) are the entries of the following mass matrices:

$$
\tilde{\mathcal{M}}_{L}^{2}=\left(\begin{array}{cc}
\tilde{m}_{L \mu \mu}^{2} & \tilde{m}_{L \mu \tau}^{2} \\
\tilde{m}_{L \mu \tau}^{2} & \tilde{m}_{L \tau \tau}^{2}
\end{array}\right), \quad \tilde{\mathcal{M}}_{R}^{2}=\left(\begin{array}{cc}
\tilde{m}_{R \mu \mu}^{2} & \tilde{m}_{R \mu \tau}^{2} \\
\tilde{m}_{R \mu \tau}^{2} & \tilde{m}_{R \tau \tau}^{2}
\end{array}\right),
$$

where we have now assumed $\tilde{m}_{L \mu \tau}^{2}, \tilde{m}_{R \mu \tau}^{2}$ to be real. The flavour states $\tilde{L}_{\mu}, \tilde{L}_{\tau}$ are related to the $\tilde{\mathcal{M}}_{L}^{2}$ eigenstates $\tilde{L}_{2}=\left(\tilde{\nu}_{2}, \tilde{\ell}_{L_{2}}\right)^{T}, \tilde{L}_{3}=\left(\tilde{\nu}_{3}, \tilde{\ell}_{L_{3}}\right)^{T}$ by the relations $\tilde{L}_{\mu}=c_{L} \tilde{L}_{2}-s_{L} \tilde{L}_{3}, \tilde{L}_{\tau}=$ $s_{L} \tilde{L}_{2}+c_{L} \tilde{L}_{3}$, where $c_{L}=\underset{\sim}{\cos } \theta_{L}, s_{L}=\underset{\sim}{\sin } \theta_{L}$. Analogous relations hold for the right-handed sleptons: $\tilde{\mu}_{R}=c_{R} \tilde{\ell}_{R_{2}}-s_{R} \tilde{\ell}_{R_{3}}, \tilde{\tau}_{R}=s_{R} \tilde{\ell}_{R_{2}}+c_{R} \tilde{\ell}_{R_{3}}$, where $\tilde{\ell}_{R_{2}}, \tilde{\ell}_{R_{3}}$ are the eigenstates of $\tilde{\mathcal{M}}_{R}^{2}$ and $c_{R}=\cos \theta_{R}, s_{R}=\sin \theta_{R}$. The mixing parameters satisfy the following relations:

$$
s_{L} c_{L}=\frac{\tilde{m}_{L \mu \tau}^{2}}{\tilde{m}_{L_{2}}^{2}-\tilde{m}_{L_{3}}^{2}}, \quad s_{R} c_{R}=\frac{\tilde{m}_{R \mu \tau}^{2}}{\tilde{m}_{R_{2}}^{2}-\tilde{m}_{R_{3}}^{2}}
$$

where $\tilde{m}_{L_{\alpha}}^{2}$ and $\tilde{m}_{R_{\alpha}}^{2}(\alpha=2,3)$ are the eigenvalues of $\tilde{\mathcal{M}}_{L}^{2}$ and $\tilde{\mathcal{M}}_{R}^{2}$, respectively.

The mass and interaction terms presented above are sufficient to perform diagrammatic calculations of the operator coefficients. As anticipated in Section 3.1, electroweak breaking effects (Higgs insertions) will be treated at lowest order. In the next sections, for each coefficient we show the relevant diagrams and display the analytical results. In diagrams with gauge boson insertions in a fermionic line we use a shorthand notation, explained in Fig. 21.

\section{A.2 Loop integrals}

The results of our diagrammatic computations will be expressed in terms of the following standard loop integrals

$$
I_{N}\left(m_{1}^{2}, \ldots, m_{N}^{2}\right) \equiv \frac{i}{\pi^{2}} \int \frac{\mathrm{d}^{4} k}{\left(k^{2}-m_{1}^{2}\right) \ldots\left(k^{2}-m_{N}^{2}\right)}=(-1)^{N+1} \int_{0}^{\infty} \frac{s \mathrm{~d} s}{\left(s+m_{1}^{2}\right) \ldots\left(s+m_{N}^{2}\right)}
$$




$$
\begin{aligned}
& \tilde{W}_{\longleftarrow}^{+}\left\{\tilde{W}^{+}=\tilde{W}_{\longleftarrow}^{+}\left\{\tilde{W}^{+}+\tilde{W}_{\longleftarrow}^{+} \tilde{W}^{-}\left\{\tilde{W}^{-} \tilde{W}^{+} \quad \stackrel{\left.\tilde{W}^{-}\right\} \tilde{W}^{+}}{\longrightarrow}=\tilde{W}^{-} \tilde{W}^{+}\left\{\tilde{W}^{+}+\tilde{W}^{-}\left\{\tilde{W}^{-} \tilde{W}^{+}\right.\right.\right.\right.\right.
\end{aligned}
$$

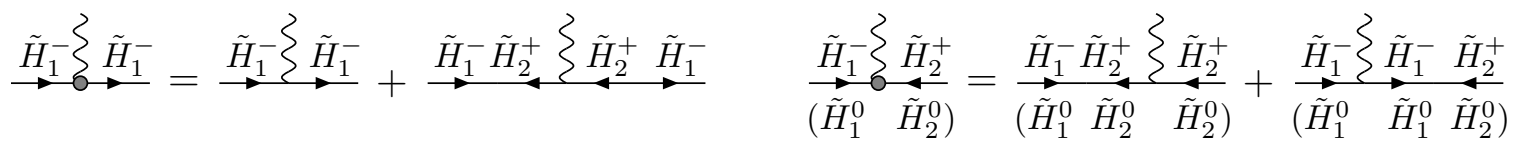

Figure 21: Shorthand notation used in some diagrams.

$$
\begin{aligned}
J_{N}\left(m_{1}^{2}, \ldots, m_{N}^{2}\right) & \equiv \frac{i}{\pi^{2}} \int \frac{k^{2} \mathrm{~d}^{4} k}{\left(k^{2}-m_{1}^{2}\right) \ldots\left(k^{2}-m_{N}^{2}\right)}=(-1)^{N} \int_{0}^{\infty} \frac{s^{2} \mathrm{~d} s}{\left(s+m_{1}^{2}\right) \ldots\left(s+m_{N}^{2}\right)} \\
K_{N}\left(m_{1}^{2}, \ldots, m_{N}^{2}\right) & \equiv \frac{i}{\pi^{2}} \int \frac{k^{4} \mathrm{~d}^{4} k}{\left(k^{2}-m_{1}^{2}\right) \ldots\left(k^{2}-m_{N}^{2}\right)}=(-1)^{N+1} \int_{0}^{\infty} \frac{s^{3} \mathrm{~d} s}{\left(s+m_{1}^{2}\right) \ldots\left(s+m_{N}^{2}\right)}
\end{aligned}
$$

It is worth recalling that the expression of a diagrammatic computation in terms of these loop integrals is not unique, since such functions are not independent (this fact is also reflected in the arbitrariness in the parametrization of diagram momenta). Several identities relating the above functions can be derived, either by simple manipulations of the integrands (i.e. the propagators) or by using obvious scaling properties (see e.g. [27]). Examples of the former kind are

$$
\begin{aligned}
I_{N}\left(m_{1}^{2}, \ldots, m_{N}^{2}\right) & =\frac{1}{m_{1}^{2}-m_{N}^{2}}\left[I_{N-1}\left(m_{1}^{2}, \ldots, m_{N-1}^{2}\right)-I_{N-1}\left(m_{2}^{2}, \ldots, m_{N}^{2}\right)\right] \\
J_{N}\left(m_{1}^{2}, \ldots, m_{N}^{2}\right) & =I_{N-1}\left(m_{1}^{2}, \ldots, m_{N-1}^{2}\right)+m_{N}^{2} I_{N}\left(m_{1}^{2}, \ldots, m_{N}^{2}\right) .
\end{aligned}
$$

An example of the latter kind is (for $N>2$ )

$$
\frac{\mathrm{d}}{\mathrm{d} \xi}\left[\xi^{N-2} I_{N}\left(\xi m_{1}^{2}, \ldots, \xi m_{N}^{2}\right)\right]_{\xi=1}=0
$$

By combining identities of both kinds, new ones can be obtained. For instance, from eqs. (87) and (88) we get

$$
\begin{aligned}
\sum_{i=1}^{N-2} J_{N+1}\left(m_{1}^{2}, \ldots, m_{i}^{2}, m_{i}^{2}, \ldots, m_{N}^{2}\right)= & -m_{N-1}^{2} I_{N+1}\left(m_{1}^{2}, \ldots, m_{N-1}^{2}, m_{N-1}^{2}, m_{N}^{2}\right) \\
& -m_{N}^{2} I_{N+1}\left(m_{1}^{2}, \ldots, m_{N-1}^{2}, m_{N}^{2}, m_{N}^{2}\right) .
\end{aligned}
$$

Specializing this equation to the case $N=4$ and $m_{3}^{2}=m_{4}^{2}$ gives a particularly useful relation

$$
J_{5}\left(m_{1}^{2}, m_{1}^{2}, m_{2}^{2}, m_{3}^{2}, m_{3}^{2}\right)+J_{5}\left(m_{1}^{2}, m_{2}^{2}, m_{2}^{2}, m_{3}^{2}, m_{3}^{2}\right)=-2 m_{3}^{2} I_{5}\left(m_{1}^{2}, m_{2}^{2}, m_{3}^{2}, m_{3}^{2}, m_{3}^{2}\right) .
$$


All such identities can be used to cast a given expression into a convenient form. One can even reduce all loop functions to a simple one ${ }^{15}$, although the latter operation may not be the best choice for numerical evaluations.

\section{A.3 Contributions to $A_{L, R}^{Z}$}
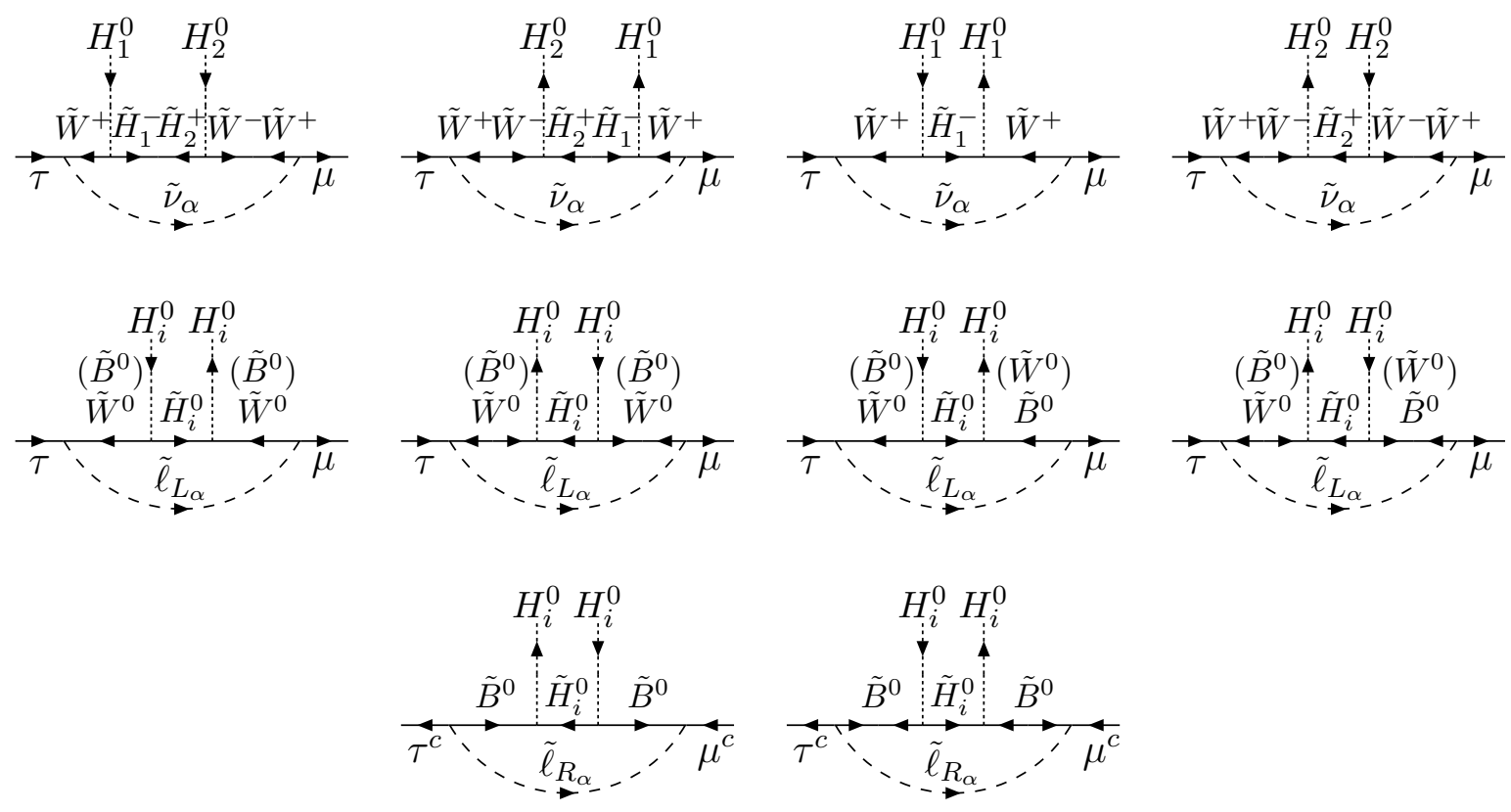

Figure 22: Diagrams that contribute to $A_{L}^{Z(a)}$ (first and second rows) and $A_{R}^{Z(a)}$ (third row).

The coefficients $A_{L, R}^{Z}$ receive contributions of three different types: $A_{L, R}^{Z}=A_{L, R}^{Z(a)}+A_{L, R}^{Z(b)}+$ $A_{L, R}^{Z(c)}$. The diagrams corresponding to $A_{L, R}^{Z(a)}$ are depicted in Fig. 22 and give:

$$
\begin{aligned}
\frac{A_{L}^{Z(a)}}{s_{L} c_{L}}= & \frac{g^{2} c_{W}^{2}}{16 \pi^{2}} \cdot \frac{1}{8}\left[-\left(2+3 c_{2 \beta}\right)\left(\mu^{2} J_{5}\left(M_{2}^{2}, M_{2}^{2}, \mu^{2}, \mu^{2}, \tilde{m}_{L_{2}}^{2}\right)+2 J_{4}\left(M_{2}^{2}, M_{2}^{2}, \mu^{2}, \tilde{m}_{L_{2}}^{2}\right)\right)\right. \\
& -\left(2-3 c_{2 \beta}\right) M_{2}^{2}\left(\mu^{2} I_{5}\left(M_{2}^{2}, M_{2}^{2}, \mu^{2}, \mu^{2}, \tilde{m}_{L_{2}}^{2}\right)-I_{4}\left(M_{2}^{2}, M_{2}^{2}, \mu^{2}, \tilde{m}_{L_{2}}^{2}\right)\right) \\
& \left.+4 s_{2 \beta} \mu M_{2} J_{5}\left(M_{2}^{2}, M_{2}^{2}, \mu^{2}, \mu^{2}, \tilde{m}_{L_{2}}^{2}\right)\right] \\
+ & \frac{g^{\prime 2} c_{W}^{2}}{16 \pi^{2}} \cdot \frac{1}{4} c_{2 \beta}\left[\mu^{2} J_{5}\left(M_{1}^{2}, M_{2}^{2}, \mu^{2}, \mu^{2}, \tilde{m}_{L_{2}}^{2}\right)+2 J_{4}\left(M_{1}^{2}, M_{2}^{2}, \mu^{2}, \tilde{m}_{L_{2}}^{2}\right)\right. \\
& \left.-M_{1} M_{2}\left(\mu^{2} I_{5}\left(M_{1}^{2}, M_{2}^{2}, \mu^{2}, \mu^{2}, \tilde{m}_{L_{2}}^{2}\right)-I_{4}\left(M_{1}^{2}, M_{2}^{2}, \mu^{2}, \tilde{m}_{L_{2}}^{2}\right)\right)\right] \\
+ & \frac{g^{\prime 2} s_{W}^{2}}{16 \pi^{2}} \cdot \frac{1}{8} c_{2 \beta}\left[-\mu^{2} J_{5}\left(M_{1}^{2}, M_{1}^{2}, \mu^{2}, \mu^{2}, \tilde{m}_{L_{2}}^{2}\right)-2 J_{4}\left(M_{1}^{2}, M_{1}^{2}, \mu^{2}, \tilde{m}_{L_{2}}^{2}\right)\right.
\end{aligned}
$$

\footnotetext{
${ }^{15}$ For example $I_{3}$, which has the explicit form $I_{3}(x, y, z)=\left(x y \log \frac{x}{y}+y z \log \frac{y}{z}+z x \log \frac{z}{x}\right) /[(x-y)(z-y)(z-x)]$.
} 


$$
\begin{aligned}
& \left.+M_{1}^{2}\left(\mu^{2} I_{5}\left(M_{1}^{2}, M_{1}^{2}, \mu^{2}, \mu^{2}, \tilde{m}_{L_{2}}^{2}\right)-I_{4}\left(M_{1}^{2}, M_{1}^{2}, \mu^{2}, \tilde{m}_{L_{2}}^{2}\right)\right)\right] \\
- & \left(L_{2} \rightarrow L_{3}\right) \\
\frac{A_{R}^{Z(a)}}{s_{R} c_{R}}= & \frac{g^{\prime 2} s_{W}^{2}}{16 \pi^{2}} \cdot \frac{1}{2} c_{2 \beta}\left[\mu^{2} J_{5}\left(M_{1}^{2}, M_{1}^{2}, \mu^{2}, \mu^{2}, \tilde{m}_{R_{2}}^{2}\right)+2 J_{4}\left(M_{1}^{2}, M_{1}^{2}, \mu^{2}, \tilde{m}_{R_{2}}^{2}\right)\right. \\
& \left.-M_{1}^{2}\left(\mu^{2} I_{5}\left(M_{1}^{2}, M_{1}^{2}, \mu^{2}, \mu^{2}, \tilde{m}_{R_{2}}^{2}\right)-I_{4}\left(M_{1}^{2}, M_{1}^{2}, \mu^{2}, \tilde{m}_{R_{2}}^{2}\right)\right)\right] \\
- & \left(R_{2} \rightarrow R_{3}\right)
\end{aligned}
$$
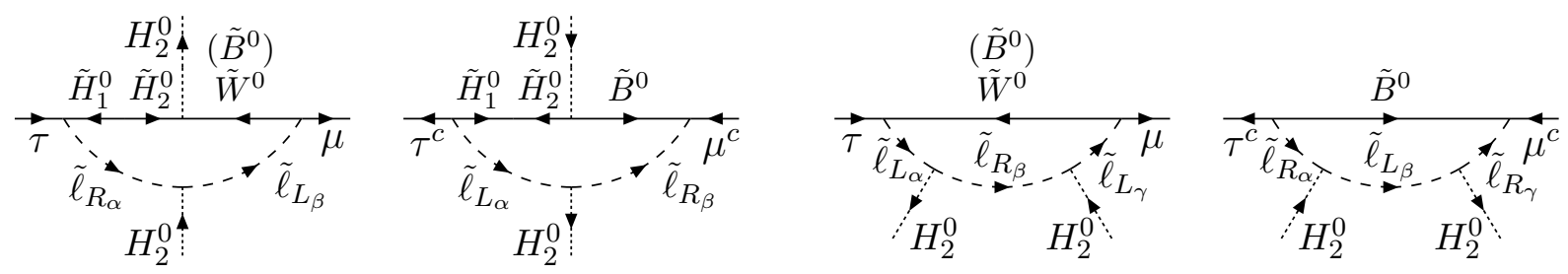

Figure 23: Diagrams that contribute to $A_{L, R}^{Z(b)}$ (left side) and $A_{L, R}^{Z(c)}$ (right side).

The diagrams corresponding to $A_{L, R}^{Z(b)}$ (Fig. 23) give:

$$
\begin{aligned}
\frac{A_{L}^{Z(b)}}{s_{L} c_{L}}= & \frac{h_{\tau}^{2} c_{W}^{2}}{16 \pi^{2}} \cdot \frac{1}{4} s_{\beta}^{2} \mu^{2}\left[s_{R}^{2}\left(J_{5}\left(M_{2}^{2}, \mu^{2}, \tilde{m}_{R_{2}}^{2}, \tilde{m}_{R_{2}}^{2}, \tilde{m}_{L_{2}}^{2}\right)+J_{5}\left(M_{2}^{2}, \mu^{2}, \mu^{2}, \tilde{m}_{R_{2}}^{2}, \tilde{m}_{L_{2}}^{2}\right)\right)\right. \\
& \left.+c_{R}^{2}\left(J_{5}\left(M_{2}^{2}, \mu^{2}, \tilde{m}_{R_{3}}^{2}, \tilde{m}_{R_{3}}^{2}, \tilde{m}_{L_{2}}^{2}\right)+J_{5}\left(M_{2}^{2}, \mu^{2}, \mu^{2}, \tilde{m}_{R_{3}}^{2}, \tilde{m}_{L_{2}}^{2}\right)\right)\right] \\
+ & \frac{h_{\tau}^{2} s_{W}^{2}}{16 \pi^{2}} \cdot \frac{1}{4} s_{\beta}^{2} \mu^{2}\left[-s_{R}^{2}\left(J_{5}\left(M_{1}^{2}, \mu^{2}, \tilde{m}_{R_{2}}^{2}, \tilde{m}_{R_{2}}^{2}, \tilde{m}_{L_{2}}^{2}\right)+J_{5}\left(M_{1}^{2}, \mu^{2}, \mu^{2}, \tilde{m}_{R_{2}}^{2}, \tilde{m}_{L_{2}}^{2}\right)\right)\right. \\
& \left.-c_{R}^{2}\left(J_{5}\left(M_{1}^{2}, \mu^{2}, \tilde{m}_{R_{3}}^{2}, \tilde{m}_{R_{3}}^{2}, \tilde{m}_{L_{2}}^{2}\right)+J_{5}\left(M_{1}^{2}, \mu^{2}, \mu^{2}, \tilde{m}_{R_{3}}^{2}, \tilde{m}_{L_{2}}^{2}\right)\right)\right] \\
\frac{A_{R}^{Z(b)}=}{s_{R} c_{R}=} & \frac{\left.h_{\tau}^{2} s_{W}^{2} \rightarrow L_{3}\right)}{16 \pi^{2}} \cdot \frac{1}{2} s_{\beta}^{2} \mu^{2}\left[-s_{L}^{2}\left(J_{5}\left(M_{1}^{2}, \mu^{2}, \tilde{m}_{L_{2}}^{2}, \tilde{m}_{L_{2}}^{2}, \tilde{m}_{R_{2}}^{2}\right)+J_{5}\left(M_{1}^{2}, \mu^{2}, \mu^{2}, \tilde{m}_{L_{2}}^{2}, \tilde{m}_{R_{2}}^{2}\right)\right)\right. \\
& \left.-c_{L}^{2}\left(J_{5}\left(M_{1}^{2}, \mu^{2}, \tilde{m}_{L_{3}}^{2}, \tilde{m}_{L_{3}}^{2}, \tilde{m}_{R_{2}}^{2}\right)+J_{5}\left(M_{1}^{2}, \mu^{2}, \mu^{2}, \tilde{m}_{L_{3}}^{2}, \tilde{m}_{R_{2}}^{2}\right)\right)\right] \\
- & \left(R_{2} \rightarrow R_{3}\right)
\end{aligned}
$$

The diagrams corresponding to $A_{L, R}^{Z(c)}$ (Fig. 23) give:

$$
\begin{aligned}
\frac{A_{L}^{Z(c)}}{s_{L} c_{L}}= & \frac{h_{\tau}^{2} c_{W}^{2}}{16 \pi^{2}} \cdot \frac{1}{4} s_{\beta}^{2} \mu^{2}\left[s _ { L } ^ { 2 } \left(s_{R}^{2} J_{5}\left(M_{2}^{2}, \tilde{m}_{R_{2}}^{2}, \tilde{m}_{R_{2}}^{2}, \tilde{m}_{L_{2}}^{2}, \tilde{m}_{L_{2}}^{2}\right)\right.\right. \\
& \left.+c_{R}^{2} J_{5}\left(M_{2}^{2}, \tilde{m}_{R_{3}}^{2}, \tilde{m}_{R_{3}}^{2}, \tilde{m}_{L_{2}}^{2}, \tilde{m}_{L_{2}}^{2}\right)\right)-c_{L}^{2}\left(s_{R}^{2} J_{5}\left(M_{2}^{2}, \tilde{m}_{R_{2}}^{2}, \tilde{m}_{R_{2}}^{2}, \tilde{m}_{L_{3}}^{2}, \tilde{m}_{L_{3}}^{2}\right)\right. \\
& \left.+c_{R}^{2} J_{5}\left(M_{2}^{2}, \tilde{m}_{R_{3}}^{2}, \tilde{m}_{R_{3}}^{2}, \tilde{m}_{L_{3}}^{2}, \tilde{m}_{L_{3}}^{2}\right)\right)-\left(s_{L}^{2}-c_{L}^{2}\right)\left(s_{R^{2}}^{2} J_{5}\left(M_{2}^{2}, \tilde{m}_{R_{2}}^{2}, \tilde{m}_{R_{2}}^{2}, \tilde{m}_{L_{2}}^{2}, \tilde{m}_{L_{3}}^{2}\right)\right. \\
& \left.\left.+c_{R}^{2} J_{5}\left(M_{2}^{2}, \tilde{m}_{R_{3}}^{2}, \tilde{m}_{R_{3}}^{2}, \tilde{m}_{L_{2}}^{2}, \tilde{m}_{L_{3}}^{2}\right)\right)\right]
\end{aligned}
$$




$$
\begin{aligned}
+ & \frac{h_{\tau}^{2} s_{W}^{2}}{16 \pi^{2}} \cdot \frac{1}{4} s_{\beta}^{2} \mu^{2}\left[s _ { L } ^ { 2 } \left(s_{R}^{2} J_{5}\left(M_{1}^{2}, \tilde{m}_{R_{2}}^{2}, \tilde{m}_{R_{2}}^{2}, \tilde{m}_{L_{2}}^{2}, \tilde{m}_{L_{2}}^{2}\right)\right.\right. \\
& \left.+c_{R}^{2} J_{5}\left(M_{1}^{2}, \tilde{m}_{R_{3}}^{2}, \tilde{m}_{R_{3}}^{2}, \tilde{m}_{L_{2}}^{2}, \tilde{m}_{L_{2}}^{2}\right)\right)-c_{L}^{2}\left(s_{R}^{2} J_{5}\left(M_{1}^{2}, \tilde{m}_{R_{2}}^{2}, \tilde{m}_{R_{2}}^{2}, \tilde{m}_{L_{3}}^{2}, \tilde{m}_{L_{3}}^{2}\right)\right. \\
& \left.+c_{R}^{2} J_{5}\left(M_{1}^{2}, \tilde{m}_{R_{3}}^{2}, \tilde{m}_{R_{3}}^{2}, \tilde{m}_{L_{3}}^{2}, \tilde{m}_{L_{3}}^{2}\right)\right)-\left(s_{L}^{2}-c_{L}^{2}\right)\left(s_{R}^{2} J_{5}\left(M_{1}^{2}, \tilde{m}_{R_{2}}^{2}, \tilde{m}_{R_{2}}^{2}, \tilde{m}_{L_{2}}^{2}, \tilde{m}_{L_{3}}^{2}\right)\right. \\
& \left.\left.+c_{R}^{2} J_{5}\left(M_{1}^{2}, \tilde{m}_{R_{3}}^{2}, \tilde{m}_{R_{3}}^{2}, \tilde{m}_{L_{2}}^{2}, \tilde{m}_{L_{3}}^{2}\right)\right)\right] \\
\frac{A_{R}^{Z(c)}}{s_{R} c_{R}}= & \frac{h_{\tau}^{2} s_{W}^{2}}{16 \pi^{2}} s_{\beta}^{2} \mu^{2}\left[-s_{R}^{2}\left(s_{L}^{2} J_{5}\left(M_{1}^{2}, \tilde{m}_{L_{2}}^{2}, \tilde{m}_{L_{2}}^{2}, \tilde{m}_{R_{2}}^{2}, \tilde{m}_{R_{2}}^{2}\right)\right.\right. \\
& \left.+c_{L}^{2} J_{5}\left(M_{1}^{2}, \tilde{m}_{L_{3}}^{2}, \tilde{m}_{L_{3}}^{2}, \tilde{m}_{R_{2}}^{2}, \tilde{m}_{R_{2}}^{2}\right)\right)+c_{R}^{2}\left(s_{L}^{2} J_{5}\left(M_{1}^{2}, \tilde{m}_{L_{2}}^{2}, \tilde{m}_{L_{2}}^{2}, \tilde{m}_{R_{3}}^{2}, \tilde{m}_{R_{3}}^{2}\right)\right. \\
& \left.+c_{L}^{2} J_{5}\left(M_{1}^{2}, \tilde{m}_{L_{3}}^{2}, \tilde{m}_{L_{3}}^{2}, \tilde{m}_{R_{3}}^{2}, \tilde{m}_{R_{3}}^{2}\right)\right)+\left(s_{R}^{2}-c_{R}^{2}\right)\left(s_{L}^{2} J_{5}\left(M_{1}^{2}, \tilde{m}_{L_{2}}^{2}, \tilde{m}_{L_{2}}^{2}, \tilde{m}_{R_{2}}^{2}, \tilde{m}_{R_{3}}^{2}\right)\right. \\
& \left.\left.+c_{L}^{2} J_{5}\left(M_{1}^{2}, \tilde{m}_{L_{3}}^{2}, \tilde{m}_{L_{3}}^{2}, \tilde{m}_{R_{2}}^{2}, \tilde{m}_{R_{3}}^{2}\right)\right)\right]
\end{aligned}
$$

\section{A.4 Contributions to $C_{L, R}^{\gamma}$}

The diagrams corresponding to $C_{L, R}^{\gamma}$ are depicted in Fig. 24 and give:

$$
\begin{aligned}
\frac{C_{L}^{\gamma}}{s_{L} c_{L}}= & \frac{g^{2}}{16 \pi^{2}} \cdot \frac{1}{12}\left[-K_{5}\left(M_{2}^{2}, \tilde{m}_{L_{2}}^{2}, \tilde{m}_{L_{2}}^{2}, \tilde{m}_{L_{2}}^{2}, \tilde{m}_{L_{2}}^{2}\right)\right. \\
& \left.-4 K_{5}\left(M_{2}^{2}, M_{2}^{2}, M_{2}^{2}, M_{2}^{2}, \tilde{m}_{L_{2}}^{2}\right)+6 M_{2}^{2} J_{5}\left(M_{2}^{2}, M_{2}^{2}, M_{2}^{2}, M_{2}^{2}, \tilde{m}_{L_{2}}^{2}\right)\right] \\
+ & \frac{g^{\prime 2}}{16 \pi^{2}} \cdot \frac{1}{12}\left[-K_{5}\left(M_{1}^{2}, \tilde{m}_{L_{2}}^{2}, \tilde{m}_{L_{2}}^{2}, \tilde{m}_{L_{2}}^{2}, \tilde{m}_{L_{2}}^{2}\right)\right]-\left(L_{2} \rightarrow L_{3}\right) \\
\frac{C_{R}^{\gamma}}{s_{R} c_{R}}= & \frac{g^{\prime 2}}{16 \pi^{2}} \cdot \frac{1}{3}\left[-K_{5}\left(M_{1}^{2}, \tilde{m}_{R_{2}}^{2}, \tilde{m}_{R_{2}}^{2}, \tilde{m}_{R_{2}}^{2}, \tilde{m}_{R_{2}}^{2}\right)\right]-\left(R_{2} \rightarrow R_{3}\right)
\end{aligned}
$$
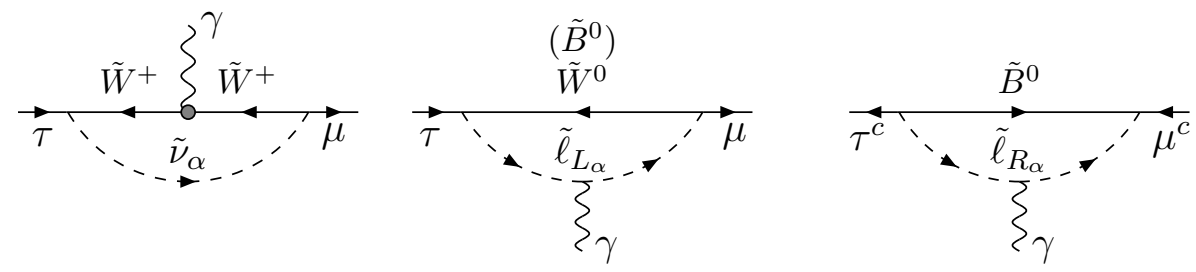

Figure 24: Diagrams that contribute to $C_{L, R}^{\gamma}$.

\section{A.5 Contributions to $C_{L, R}^{Z}$}

The diagrams corresponding to $C_{L, R}^{Z}$ are depicted in Fig. 25 and give:

$$
\frac{C_{L}^{Z}}{s_{L} c_{L}}=\frac{g^{2}}{16 \pi^{2}} \cdot \frac{1}{24}\left[\left(1+2 s_{W}^{2}\right) K_{5}\left(M_{2}^{2}, \tilde{m}_{L_{2}}^{2}, \tilde{m}_{L_{2}}^{2}, \tilde{m}_{L_{2}}^{2}, \tilde{m}_{L_{2}}^{2}\right)\right.
$$




$$
\begin{aligned}
& \left.-4 c_{W}^{2}\left(2 K_{5}\left(M_{2}^{2}, M_{2}^{2}, M_{2}^{2}, M_{2}^{2}, \tilde{m}_{L_{2}}^{2}\right)-3 M_{2}^{2} J_{5}\left(M_{2}^{2}, M_{2}^{2}, M_{2}^{2}, M_{2}^{2}, \tilde{m}_{L_{2}}^{2}\right)\right)\right] \\
+ & \frac{g^{\prime 2}}{16 \pi^{2}} \cdot \frac{1}{24}\left(-1+2 s_{W}^{2}\right) K_{5}\left(M_{1}^{2}, \tilde{m}_{L_{2}}^{2}, \tilde{m}_{L_{2}}^{2}, \tilde{m}_{L_{2}}^{2}, \tilde{m}_{L_{2}}^{2}\right)-\left(L_{2} \rightarrow L_{3}\right) \\
\frac{C_{R}^{Z}}{s_{R} c_{R}}= & \frac{g^{\prime 2}}{16 \pi^{2}} \cdot \frac{1}{3} s_{W}^{2} K_{5}\left(M_{1}^{2}, \tilde{m}_{R_{2}}^{2}, \tilde{m}_{R_{2}}^{2}, \tilde{m}_{R_{2}}^{2}, \tilde{m}_{R_{2}}^{2}\right)-\left(R_{2} \rightarrow R_{3}\right)
\end{aligned}
$$
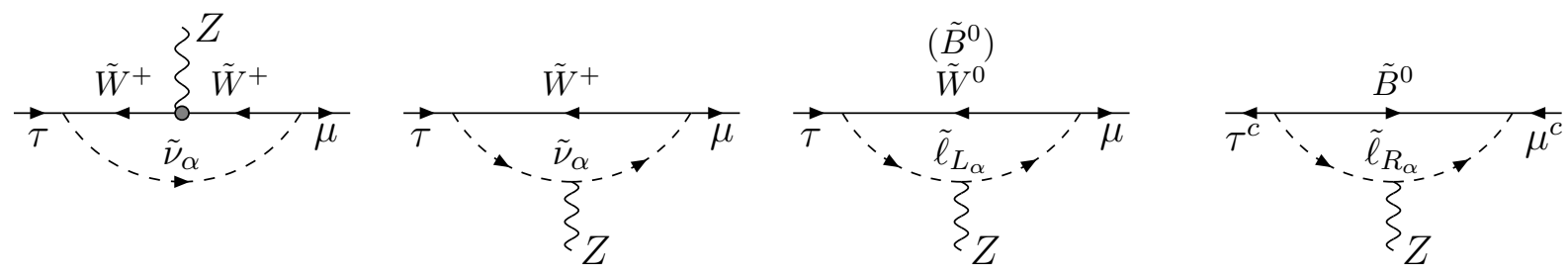

Figure 25: Diagrams that contribute to $C_{L, R}^{Z}$.

\section{A.6 Contributions to $D_{L, R}^{\gamma}$}

The coefficients $D_{L, R}^{\gamma}$ receive contributions of three different types: $D_{L, R}^{\gamma}=D_{L, R}^{\gamma(a)}+D_{L, R}^{\gamma(b)}+D_{L, R}^{\gamma(c)}$.

The diagrams corresponding to $D_{L, R}^{\gamma(a)}$ are depicted in Fig. 26 and give:

$$
\begin{aligned}
& \frac{D_{L}^{\gamma(a)}}{s_{L} c_{L}}=\frac{g^{2}}{16 \pi^{2}} \cdot \frac{1}{4}\left[\tilde{m}_{L_{2}}^{2} J_{5}\left(M_{2}^{2}, \tilde{m}_{L_{2}}^{2}, \tilde{m}_{L_{2}}^{2}, \tilde{m}_{L_{2}}^{2}, \tilde{m}_{L_{2}}^{2}\right)-2 M_{2}^{2} J_{5}\left(M_{2}^{2}, M_{2}^{2}, M_{2}^{2}, M_{2}^{2}, \tilde{m}_{L_{2}}^{2}\right)\right] \\
& +\frac{g^{\prime 2}}{16 \pi^{2}} \cdot \frac{1}{4} \tilde{m}_{L_{2}}^{2} J_{5}\left(M_{1}^{2}, \tilde{m}_{L_{2}}^{2}, \tilde{m}_{L_{2}}^{2}, \tilde{m}_{L_{2}}^{2}, \tilde{m}_{L_{2}}^{2}\right)-\left(L_{2} \rightarrow L_{3}\right) \\
& \frac{D_{R}^{\gamma(a)}}{s_{R} c_{R}}=\frac{g^{\prime 2}}{16 \pi^{2}} \tilde{m}_{R_{2}}^{2} J_{5}\left(M_{1}^{2}, \tilde{m}_{R_{2}}^{2}, \tilde{m}_{R_{2}}^{2}, \tilde{m}_{R_{2}}^{2}, \tilde{m}_{R_{2}}^{2}\right)-\left(R_{2} \rightarrow R_{3}\right)
\end{aligned}
$$
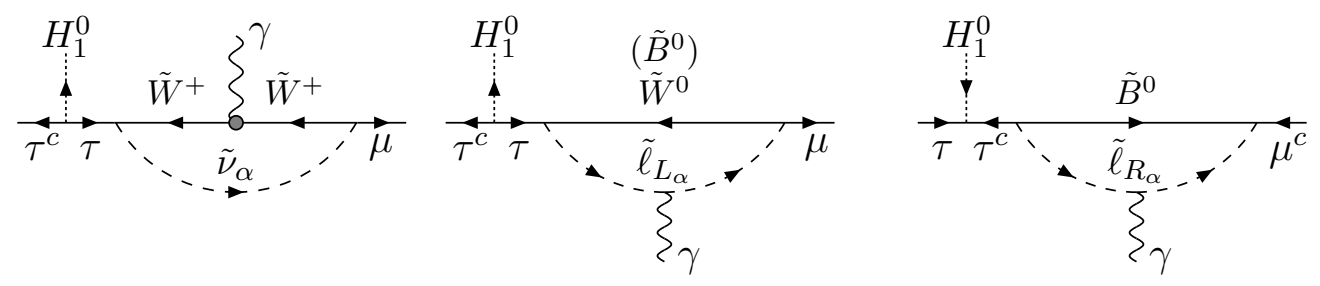

Figure 26: Diagrams that contribute to $D_{L, R}^{\gamma(a)}$.

The diagrams corresponding to $D_{L, R}^{\gamma(b)}$ are depicted in Fig. 27 and give:

$\frac{D_{L}^{\gamma(b)}}{s_{L} c_{L}}=\frac{g^{2}}{16 \pi^{2}} \cdot \frac{1}{2}\left[-\tilde{m}_{L_{2}}^{2} J_{5}\left(M_{2}^{2}, \mu^{2}, \tilde{m}_{L_{2}}^{2}, \tilde{m}_{L_{2}}^{2}, \tilde{m}_{L_{2}}^{2}\right)-2 \tilde{m}_{L_{2}}^{4} I_{5}\left(M_{2}^{2}, \mu^{2}, \tilde{m}_{L_{2}}^{2}, \tilde{m}_{L_{2}}^{2}, \tilde{m}_{L_{2}}^{2}\right)\right.$ 


$$
\begin{aligned}
& +M_{2} \mu \tan \beta\left(\tilde{m}_{L_{2}}^{2} I_{5}\left(M_{2}^{2}, \mu^{2}, \tilde{m}_{L_{2}}^{2}, \tilde{m}_{L_{2}}^{2}, \tilde{m}_{L_{2}}^{2}\right)+2 J_{5}\left(M_{2}^{2}, M_{2}^{2}, \mu^{2}, \mu^{2}, \tilde{m}_{L_{2}}^{2}\right)\right. \\
& \left.\left.+2 J_{5}\left(M_{2}^{2}, M_{2}^{2}, M_{2}^{2}, \mu^{2}, \tilde{m}_{L_{2}}^{2}\right)+2 J_{5}\left(M_{2}^{2}, \mu^{2}, \mu^{2}, \mu^{2}, \tilde{m}_{L_{2}}^{2}\right)\right)\right] \\
+ & \frac{g^{\prime 2}}{16 \pi^{2}} \frac{\tilde{m}_{L_{2}}^{2}}{2}\left[J_{5}\left(M_{1}^{2}, \mu^{2}, \tilde{m}_{L_{2}}^{2}, \tilde{m}_{L_{2}}^{2}, \tilde{m}_{L_{2}}^{2}\right)-M_{1} \mu \tan \beta I_{5}\left(M_{1}^{2}, \mu^{2}, \tilde{m}_{L_{2}}^{2}, \tilde{m}_{L_{2}}^{2}, \tilde{m}_{L_{2}}^{2}\right)\right] \\
- & \left(L_{2} \rightarrow L_{3}\right) \\
\frac{D_{R}^{\gamma(b)}}{s_{R} c_{R}}= & \frac{g^{\prime 2}}{16 \pi^{2}} \tilde{m}_{R_{2}}^{2}\left[-J_{5}\left(M_{1}^{2}, \mu^{2}, \tilde{m}_{R_{2}}^{2}, \tilde{m}_{R_{2}}^{2}, \tilde{m}_{R_{2}}^{2}\right)+M_{1} \mu \tan \beta I_{5}\left(M_{1}^{2}, \mu^{2}, \tilde{m}_{R_{2}}^{2}, \tilde{m}_{R_{2}}^{2}, \tilde{m}_{R_{2}}^{2}\right)\right] \\
- & \left(R_{2} \rightarrow R_{3}\right)
\end{aligned}
$$
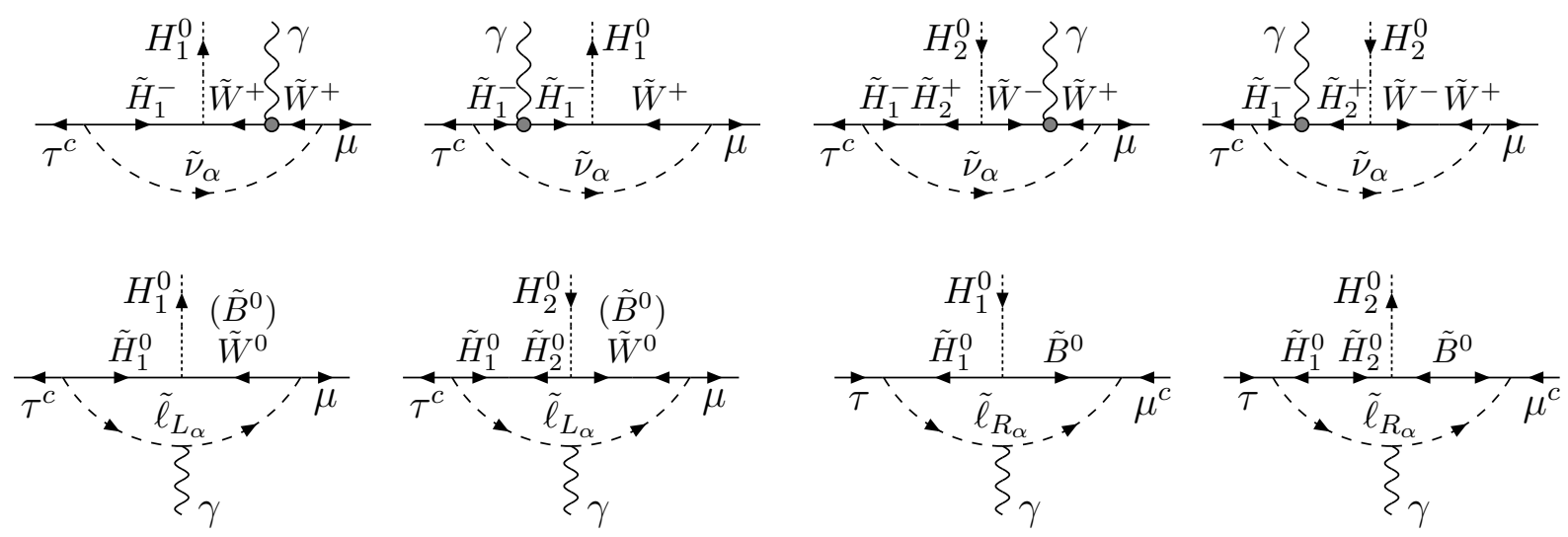

Figure 27: Diagrams that contribute to $D_{L, R}^{\gamma(b)}$.

The diagrams corresponding to $D_{L, R}^{\gamma(c)}$ are depicted in Fig. 28 and give:

$$
\begin{aligned}
D_{L}^{\gamma(c)}= & -\frac{g^{\prime 2}}{16 \pi^{2}} M_{1}^{3} \times \\
& \left\{\left[s_{L} c_{L}\left(s_{R}^{2}\left[A_{\tau}+\mu \tan \beta\right]+s_{R} c_{R} A_{\mu \tau}^{R}\right)+c_{L}^{2} s_{R}^{2} A_{\mu \tau}^{L}\right] I_{5}\left(M_{1}^{2}, M_{1}^{2}, M_{1}^{2}, \tilde{m}_{L_{2}}^{2}, \tilde{m}_{R_{2}}^{2}\right)\right. \\
- & {\left[s_{L} c_{L}\left(s_{R}^{2}\left[A_{\tau}+\mu \tan \beta\right]+s_{R} c_{R} A_{\mu \tau}^{R}\right)-s_{L}^{2} s_{R}^{2} A_{\mu \tau}^{L}\right] I_{5}\left(M_{1}^{2}, M_{1}^{2}, M_{1}^{2}, \tilde{m}_{L_{3}}^{2}, \tilde{m}_{R_{2}}^{2}\right) } \\
+ & {\left[s_{L} c_{L}\left(c_{R}^{2}\left[A_{\tau}+\mu \tan \beta\right]-s_{R} c_{R} A_{\mu \tau}^{R}\right)+c_{L}^{2} c_{R}^{2} A_{\mu \tau}^{L}\right] I_{5}\left(M_{1}^{2}, M_{1}^{2}, M_{1}^{2}, \tilde{m}_{L_{2}}^{2}, \tilde{m}_{R_{3}}^{2}\right) } \\
- & {\left.\left[s_{L} c_{L}\left(c_{R}^{2}\left[A_{\tau}+\mu \tan \beta\right]-s_{R} c_{R} A_{\mu \tau}^{R}\right)-s_{L}^{2} c_{R}^{2} A_{\mu \tau}^{L}\right] I_{5}\left(M_{1}^{2}, M_{1}^{2}, M_{1}^{2}, \tilde{m}_{L_{3}}^{2}, \tilde{m}_{R_{3}}^{2}\right)\right\} } \\
D_{R}^{\gamma(c)}= & -\frac{g^{\prime 2}}{16 \pi^{2}} M_{1}^{3} \times \\
& \left\{\left[s_{R} c_{R}\left(s_{L}^{2}\left[A_{\tau}+\mu \tan \beta\right]+s_{L} c_{L} A_{\mu \tau}^{L}\right)+c_{R}^{2} s_{L}^{2} A_{\mu \tau}^{R}\right] I_{5}\left(M_{1}^{2}, M_{1}^{2}, M_{1}^{2}, \tilde{m}_{L_{2}}^{2}, \tilde{m}_{R_{2}}^{2}\right)\right. \\
& -\left[s_{R} c_{R}\left(s_{L}^{2}\left[A_{\tau}+\mu \tan \beta\right]+s_{L} c_{L} A_{\mu \tau}^{L}\right)-s_{R}^{2} s_{L}^{2} A_{\mu \tau}^{R}\right] I_{5}\left(M_{1}^{2}, M_{1}^{2}, M_{1}^{2}, \tilde{m}_{L_{2}}^{2}, \tilde{m}_{R_{3}}^{2}\right) \\
& +\left[s_{R} c_{R}\left(c_{L}^{2}\left[A_{\tau}+\mu \tan \beta\right]-s_{L} c_{L} A_{\mu \tau}^{L}\right)+c_{R}^{2} c_{L}^{2} A_{\mu \tau}^{R}\right] I_{5}\left(M_{1}^{2}, M_{1}^{2}, M_{1}^{2}, \tilde{m}_{L_{3}}^{2}, \tilde{m}_{R_{2}}^{2}\right)
\end{aligned}
$$




$$
\left.-\left[s_{R} c_{R}\left(c_{L}^{2}\left[A_{\tau}+\mu \tan \beta\right]-s_{L} c_{L} A_{\mu \tau}^{L}\right)-s_{R}^{2} c_{L}^{2} A_{\mu \tau}^{R}\right] I_{5}\left(M_{1}^{2}, M_{1}^{2}, M_{1}^{2}, \tilde{m}_{L_{3}}^{2}, \tilde{m}_{R_{3}}^{2}\right)\right\}
$$
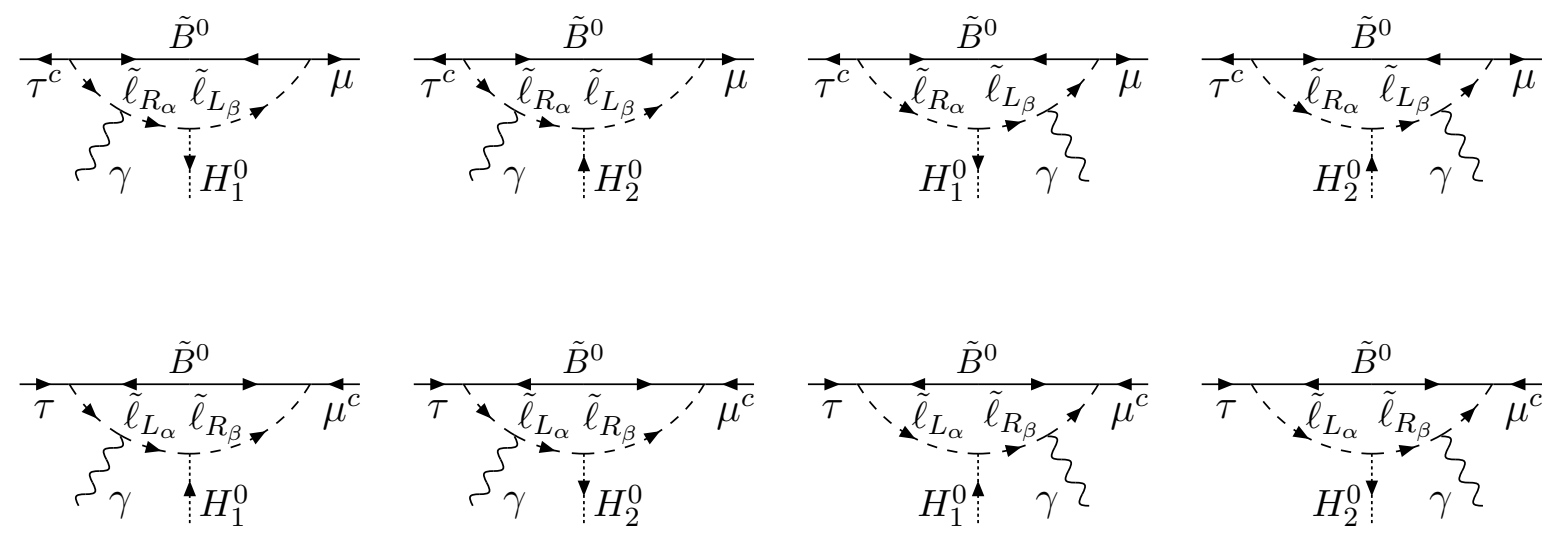

Figure 28: Diagrams that contribute to $D_{L}^{\gamma(c)}$ (first row) and $D_{R}^{\gamma(c)}$ (second row).

\section{A.7 Contributions to $D_{L, R}^{Z}$}

The leading contributions to $D_{L, R}^{Z}$ are of two types: $D_{L, R}^{Z}=D_{L, R}^{Z(b)}+D_{L, R}^{Z(c)}$. The diagrams corresponding to $D_{L, R}^{Z(a)}$ are depicted in Fig. 29 and give:

$$
\begin{aligned}
\frac{D_{L}^{Z(b)}}{s_{L} c_{L}}= & \frac{g^{2}}{16 \pi^{2}} \cdot \frac{1}{8} M_{2} \mu \tan \beta\left[-2\left(1+2 s_{W}^{2}\right) \tilde{m}_{L_{2}}^{2} I_{5}\left(M_{2}^{2}, \mu^{2}, \tilde{m}_{L_{2}}^{2}, \tilde{m}_{L_{2}}^{2}, \tilde{m}_{L_{2}}^{2}\right)\right. \\
& +2\left(1-4 s_{W}^{2}\right) J_{5}\left(M_{2}^{2}, \mu^{2}, \mu^{2}, \mu^{2}, \tilde{m}_{L_{2}}^{2}\right)+8 c_{W}^{2} J_{5}\left(M_{2}^{2}, M_{2}^{2}, M_{2}^{2}, \mu^{2}, \tilde{m}_{L_{2}}^{2}\right) \\
& \left.+\left(5-8 s_{W}^{2}\right) J_{5}\left(M_{2}^{2}, M_{2}^{2}, \mu^{2}, \mu^{2}, \tilde{m}_{L_{2}}^{2}\right)\right] \\
+ & \frac{g^{\prime 2}}{16 \pi^{2}} \cdot \frac{1}{8} M_{1} \mu \tan \beta\left[-2\left(1-2 s_{W}^{2}\right) \tilde{m}_{L_{2}}^{2} I_{5}\left(M_{1}^{2}, \mu^{2}, \tilde{m}_{L_{2}}^{2}, \tilde{m}_{L_{2}}^{2}, \tilde{m}_{L_{2}}^{2}\right)\right. \\
& \left.+2 J_{5}\left(M_{1}^{2}, \mu^{2}, \mu^{2}, \mu^{2}, \tilde{m}_{L_{2}}^{2}\right)+J_{5}\left(M_{1}^{2}, M_{1}^{2}, \mu^{2}, \mu^{2}, \tilde{m}_{L_{2}}^{2}\right)\right]-\left(L_{2} \rightarrow L_{3}\right) \\
\frac{D_{R}^{Z(b)}=}{s_{R} c_{R}}= & \frac{g^{\prime 2}}{16 \pi^{2}} \cdot \frac{1}{4} M_{1} \mu \tan \beta\left[-4 s_{W}^{2} \tilde{m}_{R_{2}}^{2} I_{5}\left(M_{1}^{2}, \mu^{2}, \tilde{m}_{R_{2}}^{2}, \tilde{m}_{R_{2}}^{2}, \tilde{m}_{R_{2}}^{2}\right)\right. \\
& \left.+2 J_{5}\left(M_{1}^{2}, \mu^{2}, \mu^{2}, \mu^{2}, \tilde{m}_{R_{2}}^{2}\right)+J_{5}\left(M_{1}^{2}, M_{1}^{2}, \mu^{2}, \mu^{2}, \tilde{m}_{R_{2}}^{2}\right)\right]-\left(R_{2} \rightarrow R_{3}\right)
\end{aligned}
$$

The diagrams corresponding to $D_{L, R}^{Z(c)}$ are depicted in Fig. 30 and give:

$$
\begin{aligned}
\frac{D_{L}^{Z(c)}}{s_{L} c_{L}}= & \frac{g^{\prime 2}}{16 \pi^{2}} \cdot \frac{1}{4} M_{1} \mu \tan \beta\left[( 1 - 2 s _ { W } ^ { 2 } ) \left(s_{R}^{2} J_{5}\left(M_{1}^{2}, M_{1}^{2}, \tilde{m}_{L_{2}}^{2}, \tilde{m}_{L_{2}}^{2}, \tilde{m}_{R_{2}}^{2}\right)\right.\right. \\
& \left.+c_{R}^{2} J_{5}\left(M_{1}^{2}, M_{1}^{2}, \tilde{m}_{L_{2}}^{2}, \tilde{m}_{L_{2}}^{2}, \tilde{m}_{R_{3}}^{2}\right)\right)-2 s_{W}^{2}\left(s_{R}^{2} J_{5}\left(M_{1}^{2}, M_{1}^{2}, \tilde{m}_{L_{2}}^{2}, \tilde{m}_{R_{2}}^{2}, \tilde{m}_{R_{2}}^{2}\right)\right.
\end{aligned}
$$



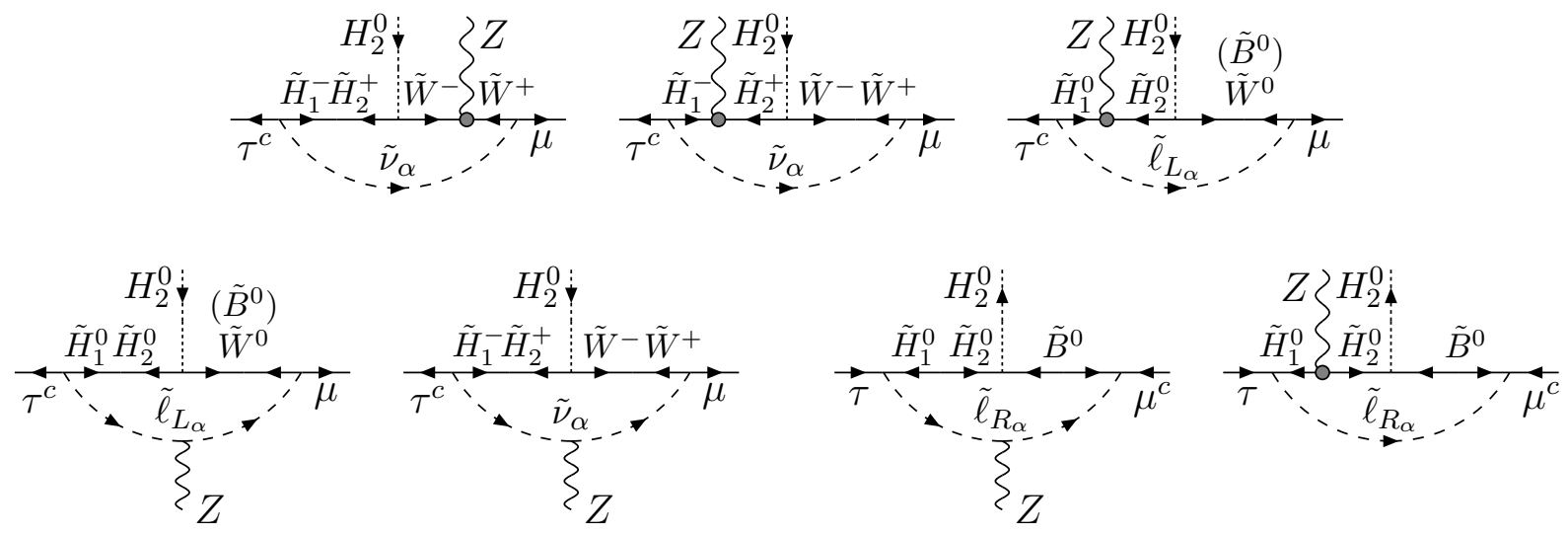

Figure 29: Diagrams that contribute to $D_{L, R}^{Z(b)}$.

$$
\begin{aligned}
& \left.\left.+c_{R}^{2} J_{5}\left(M_{1}^{2}, M_{1}^{2}, \tilde{m}_{L_{2}}^{2}, \tilde{m}_{R_{3}}^{2}, \tilde{m}_{R_{3}}^{2}\right)\right)\right]-\left(L_{2} \rightarrow L_{3}\right) \\
& \frac{D_{R}^{Z(c)}}{s_{R} c_{R}}=\frac{g^{\prime 2}}{16 \pi^{2}} \cdot \frac{1}{4} M_{1} \mu \tan \beta\left[( 1 - 2 s _ { W } ^ { 2 } ) \left(s_{L}^{2} J_{5}\left(M_{1}^{2}, M_{1}^{2}, \tilde{m}_{L_{2}}^{2}, \tilde{m}_{L_{2}}^{2}, \tilde{m}_{R_{2}}^{2}\right)\right.\right. \\
& \left.+c_{L}^{2} J_{5}\left(M_{1}^{2}, M_{1}^{2}, \tilde{m}_{L_{3}}^{2}, \tilde{m}_{L_{3}}^{2}, \tilde{m}_{R_{2}}^{2}\right)\right)-2 s_{W}^{2}\left(s_{L}^{2} J_{5}\left(M_{1}^{2}, M_{1}^{2}, \tilde{m}_{L_{2}}^{2}, \tilde{m}_{R_{2}}^{2}, \tilde{m}_{R_{2}}^{2}\right)\right. \\
& \left.\left.+c_{L}^{2} J_{5}\left(M_{1}^{2}, M_{1}^{2}, \tilde{m}_{L_{3}}^{2}, \tilde{m}_{R_{2}}^{2}, \tilde{m}_{R_{2}}^{2}\right)\right)\right]-\left(R_{2} \rightarrow R_{3}\right)
\end{aligned}
$$

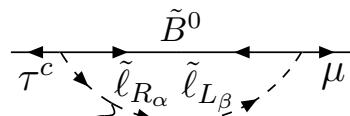

$$
\begin{aligned}
& \int_{Z} H_{2}^{0}
\end{aligned}
$$
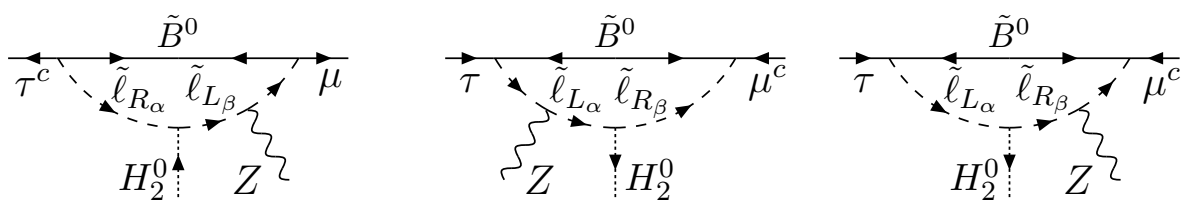

Figure 30: Diagrams that contribute to $D_{L, R}^{Z(c)}$.

\section{A.8 Contributions to $B_{L, R}^{f_{L, R}}$}

Contributions to $B_{L, R}^{f_{L, R}}$ arise from the diagrams in Fig. 31.

For $f \neq \mu, \tau$ :

$$
\begin{aligned}
\frac{B_{L}^{f_{L}}}{s_{L} c_{L}}= & \frac{g^{4}}{16 \pi^{2}} \cdot \frac{1}{16}\left[-\left(1+4 \delta_{T_{f_{L}}^{3},-\frac{1}{2}}\right) J_{4}\left(M_{2}^{2}, M_{2}^{2}, \tilde{m}_{L_{2}}^{2}, \tilde{m}_{\tilde{f}_{L}}^{2}\right)\right. \\
& \left.-2\left(1+4 \delta_{T_{f_{L}}^{3}, \frac{1}{2}}\right) M_{2}^{2} I_{4}\left(M_{2}^{2}, M_{2}^{2}, \tilde{m}_{L_{2}}^{2}, \tilde{m}_{\tilde{f}_{L}}^{2}\right)\right] \\
+ & \frac{g^{2} g^{\prime 2}}{16 \pi^{2}} \cdot \frac{1}{2}\left(-T_{f_{L}}^{3} Y_{f_{L}}\right)\left[J_{4}\left(M_{1}^{2}, M_{2}^{2}, \tilde{m}_{L_{2}}^{2}, \tilde{m}_{\tilde{f}_{L}}^{2}\right)+2 M_{1} M_{2} I_{4}\left(M_{1}^{2}, M_{2}^{2}, \tilde{m}_{L_{2}}^{2}, \tilde{m}_{\tilde{f}_{L}}^{2}\right)\right]
\end{aligned}
$$



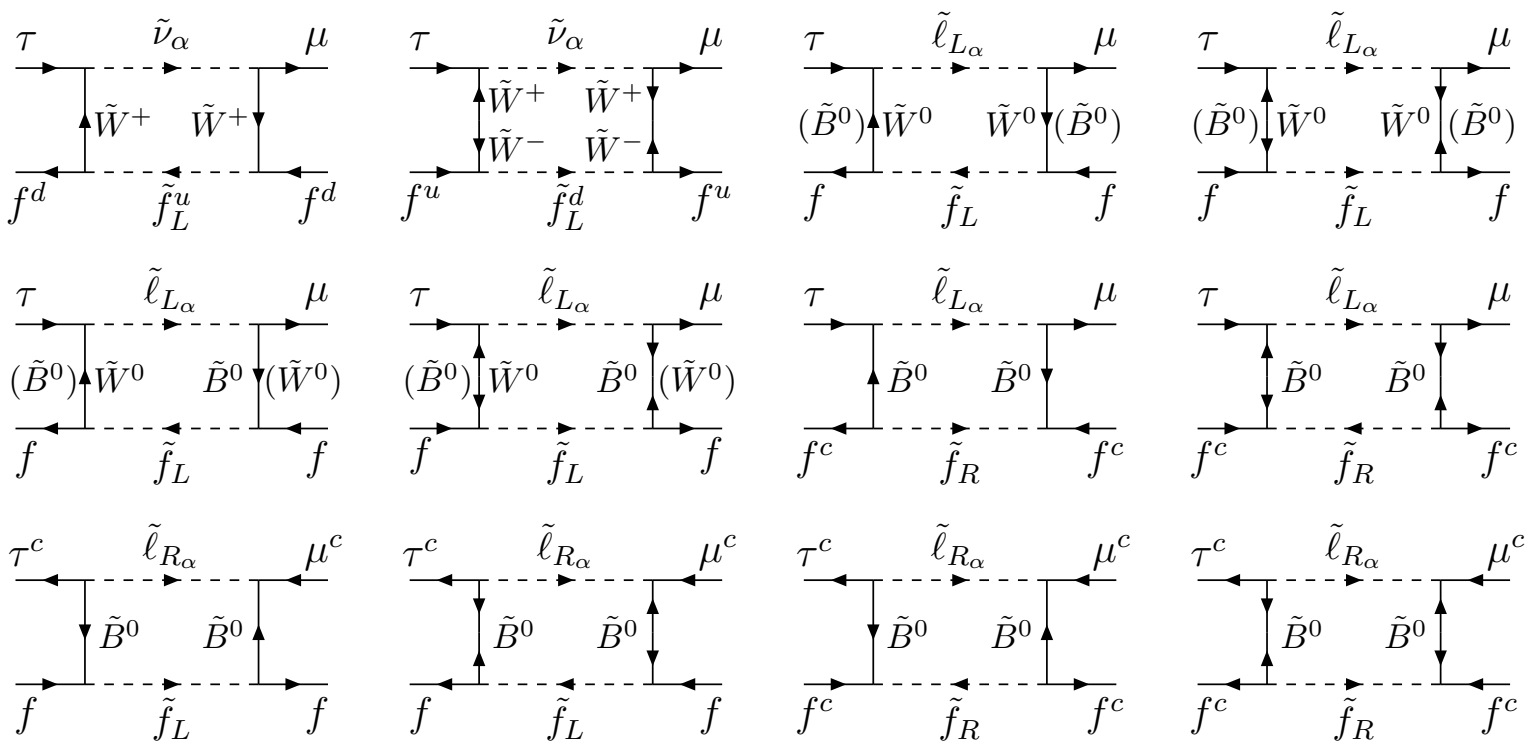

Figure 31: Diagrams that contribute to $B_{L}^{f_{L, R}}$ (first and second rows) and $B_{R}^{f_{L, R}}$ (third row). In the case of $B_{L, R}^{\mu_{L, R}}$, i.e. $f=f^{d}=\mu, f^{c}=\mu^{c}$, the following replacements are implied: $\tilde{f}_{L}^{u} \rightarrow \tilde{\nu}_{\beta}, \tilde{f}_{L} \rightarrow \tilde{\ell}_{L_{\beta}}, \tilde{f}_{R} \rightarrow \tilde{\ell}_{R_{\beta}}$.

$$
\begin{aligned}
& +\frac{g^{\prime 4}}{16 \pi^{2}} \cdot \frac{1}{4}\left(-Y_{f_{L}}^{2}\right)\left[J_{4}\left(M_{1}^{2}, M_{1}^{2}, \tilde{m}_{L_{2}}^{2}, \tilde{m}_{\tilde{f}_{L}}^{2}\right)+2 M_{1}^{2} I_{4}\left(M_{1}^{2}, M_{1}^{2}, \tilde{m}_{L_{2}}^{2}, \tilde{m}_{\tilde{f}_{L}}^{2}\right)\right] \\
& -\left(L_{2} \rightarrow L_{3}\right) \\
\frac{B_{L}^{f_{R}}}{s_{L} c_{L}} & =\frac{g^{\prime 4}}{16 \pi^{2}} \cdot \frac{1}{4} Y_{f_{R}}^{2}\left[J_{4}\left(M_{1}^{2}, M_{1}^{2}, \tilde{m}_{L_{2}}^{2}, \tilde{m}_{\tilde{f}_{R}}^{2}\right)+2 M_{1}^{2} I_{4}\left(M_{1}^{2}, M_{1}^{2}, \tilde{m}_{L_{2}}^{2}, \tilde{m}_{\tilde{f}_{R}}^{2}\right)\right] \\
& -\left(L_{2} \rightarrow L_{3}\right) \\
\frac{B_{R}^{f_{L}}}{s_{R} c_{R}} & =\frac{g^{\prime 4}}{16 \pi^{2}} Y_{f_{L}}^{2}\left[J_{4}\left(M_{1}^{2}, M_{1}^{2}, \tilde{m}_{R_{2}}^{2}, \tilde{m}_{\tilde{f}_{L}}^{2}\right)+2 M_{1}^{2} I_{4}\left(M_{1}^{2}, M_{1}^{2}, \tilde{m}_{R_{2}}^{2}, \tilde{m}_{\tilde{f}_{L}}^{2}\right)\right] \\
& -\left(R_{2} \rightarrow R_{3}\right) \\
\frac{B_{R}^{f_{R}}}{s_{R} c_{R}} & =\frac{g^{\prime 4}}{16 \pi^{2}}\left(-Y_{f_{R}}^{2}\right)\left[J_{4}\left(M_{1}^{2}, M_{1}^{2}, \tilde{m}_{R_{2}}^{2}, \tilde{m}_{\tilde{f}_{R}}^{2}\right)+2 M_{1}^{2} I_{4}\left(M_{1}^{2}, M_{1}^{2}, \tilde{m}_{R_{2}}^{2}, \tilde{m}_{\tilde{f}_{R}}^{2}\right)\right] \\
& -\left(R_{2} \rightarrow R_{3}\right)
\end{aligned}
$$

For $f=\mu$ :

$$
\begin{aligned}
\frac{B_{L}^{\mu_{L}}}{s_{L} c_{L}}= & \frac{g^{4}}{16 \pi^{2}} \cdot \frac{1}{16}\left[-c_{L}^{2}\left(5 J_{4}\left(M_{2}^{2}, M_{2}^{2}, \tilde{m}_{L_{2}}^{2}, \tilde{m}_{L_{2}}^{2}\right)+2 M_{2}^{2} I_{4}\left(M_{2}^{2}, M_{2}^{2}, \tilde{m}_{L_{2}}^{2}, \tilde{m}_{L_{2}}^{2}\right)\right)\right. \\
& +s_{L}^{2}\left(5 J_{4}\left(M_{2}^{2}, M_{2}^{2}, \tilde{m}_{L_{3}}^{2}, \tilde{m}_{L_{3}}^{2}\right)+2 M_{2}^{2} I_{4}\left(M_{2}^{2}, M_{2}^{2}, \tilde{m}_{L_{3}}^{2}, \tilde{m}_{L_{3}}^{2}\right)\right) \\
& \left.+\left(c_{L}^{2}-s_{L}^{2}\right)\left(5 J_{4}\left(M_{2}^{2}, M_{2}^{2}, \tilde{m}_{L_{2}}^{2}, \tilde{m}_{L_{3}}^{2}\right)+2 M_{2}^{2} I_{4}\left(M_{2}^{2}, M_{2}^{2}, \tilde{m}_{L_{2}}^{2}, \tilde{m}_{L_{3}}^{2}\right)\right)\right] \\
+ & \frac{g^{2} g^{\prime 2}}{16 \pi^{2}} \cdot \frac{1}{8}\left[-c_{L}^{2}\left(J_{4}\left(M_{1}^{2}, M_{2}^{2}, \tilde{m}_{L_{2}}^{2}, \tilde{m}_{L_{2}}^{2}\right)+2 M_{1} M_{2} I_{4}\left(M_{1}^{2}, M_{2}^{2}, \tilde{m}_{L_{2}}^{2}, \tilde{m}_{L_{2}}^{2}\right)\right)\right. \\
& +s_{L}^{2}\left(J_{4}\left(M_{1}^{2}, M_{2}^{2}, \tilde{m}_{L_{3}}^{2}, \tilde{m}_{L_{3}}^{2}\right)+2 M_{1} M_{2} I_{4}\left(M_{1}^{2}, M_{2}^{2}, \tilde{m}_{L_{3}}^{2}, \tilde{m}_{L_{3}}^{2}\right)\right)
\end{aligned}
$$




$$
\begin{aligned}
& \left.+\left(c_{L}^{2}-s_{L}^{2}\right)\left(J_{4}\left(M_{1}^{2}, M_{2}^{2}, \tilde{m}_{L_{2}}^{2}, \tilde{m}_{L_{3}}^{2}\right)+2 M_{1} M_{2} I_{4}\left(M_{1}^{2}, M_{2}^{2}, \tilde{m}_{L_{2}}^{2}, \tilde{m}_{L_{3}}^{2}\right)\right)\right] \\
+ & \frac{g^{\prime 4}}{16 \pi^{2}} \cdot \frac{1}{16}\left[-c_{L}^{2}\left(J_{4}\left(M_{1}^{2}, M_{1}^{2}, \tilde{m}_{L_{2}}^{2}, \tilde{m}_{L_{2}}^{2}\right)+2 M_{1}^{2} I_{4}\left(M_{1}^{2}, M_{1}^{2}, \tilde{m}_{L_{2}}^{2}, \tilde{m}_{L_{2}}^{2}\right)\right)\right. \\
& +s_{L}^{2}\left(J_{4}\left(M_{1}^{2}, M_{1}^{2}, \tilde{m}_{L_{3}}^{2}, \tilde{m}_{L_{3}}^{2}\right)+2 M_{1}^{2} I_{4}\left(M_{1}^{2}, M_{1}^{2}, \tilde{m}_{L_{3}}^{2}, \tilde{m}_{L_{3}}^{2}\right)\right) \\
& \left.+\left(c_{L}^{2}-s_{L}^{2}\right)\left(J_{4}\left(M_{1}^{2}, M_{1}^{2}, \tilde{m}_{L_{2}}^{2}, \tilde{m}_{L_{3}}^{2}\right)+2 M_{1}^{2} I_{4}\left(M_{1}^{2}, M_{1}^{2}, \tilde{m}_{L_{2}}^{2}, \tilde{m}_{L_{3}}^{2}\right)\right)\right] \\
\frac{B_{L}^{\mu_{R}}}{s_{L} c_{L}}= & \frac{g^{\prime 4}}{16 \pi^{2}} \cdot \frac{1}{4}\left[c_{R}^{2}\left(J_{4}\left(M_{1}^{2}, M_{1}^{2}, \tilde{m}_{L_{2}}^{2}, \tilde{m}_{R_{2}}^{2}\right)+2 M_{1}^{2} I_{4}\left(M_{1}^{2}, M_{1}^{2}, \tilde{m}_{L_{2}}^{2}, \tilde{m}_{R_{2}}^{2}\right)\right)\right. \\
& \left.+s_{R}^{2}\left(J_{4}\left(M_{1}^{2}, M_{1}^{2}, \tilde{m}_{L_{2}}^{2}, \tilde{m}_{R_{3}}^{2}\right)+2 M_{1}^{2} I_{4}\left(M_{1}^{2}, M_{1}^{2}, \tilde{m}_{L_{2}}^{2}, \tilde{m}_{R_{3}}^{2}\right)\right)\right] \\
\frac{B_{R}^{\mu_{L}}}{s_{R} c_{R}}= & \frac{g^{\prime 4}}{16 \pi^{2}} \cdot \frac{1}{4}\left[c_{L}^{2}\left(J_{4}\left(M_{1}^{2}, M_{1}^{2}, \tilde{m}_{L_{2}}^{2}, \tilde{m}_{R_{2}}^{2}\right)+2 M_{1}^{2} I_{4}\left(M_{1}^{2}, M_{1}^{2}, \tilde{m}_{L_{2}}^{2}, \tilde{m}_{R_{2}}^{2}\right)\right)\right. \\
& \left.+s_{L}^{2}\left(J_{4}\left(M_{1}^{2}, M_{1}^{2}, \tilde{m}_{L_{3}}^{2}, \tilde{m}_{R_{2}}^{2}\right)+2 M_{1}^{2} I_{4}\left(M_{1}^{2}, M_{1}^{2}, \tilde{m}_{L_{3}}^{2}, \tilde{m}_{R_{2}}^{2}\right)\right)\right] \\
- & \left(R_{2} \rightarrow R_{3}\right) \\
\frac{B_{R}^{\prime 4}}{s_{R} c_{R}}= & \frac{g^{\prime}}{16 \pi^{2}}\left[-c_{R}^{2}\left(J_{4}\left(M_{1}^{2}, M_{1}^{2}, \tilde{m}_{R_{2}}^{2}, \tilde{m}_{R_{2}}^{2}\right)+2 M_{1}^{2} I_{4}\left(M_{1}^{2}, M_{1}^{2}, \tilde{m}_{R_{2}}^{2}, \tilde{m}_{R_{2}}^{2}\right)\right)\right. \\
& +s_{R}^{2}\left(J_{4}\left(M_{1}^{2}, M_{1}^{2}, \tilde{m}_{R_{3}}^{2}, \tilde{m}_{R_{3}}^{2}\right)+2 M_{1}^{2} I_{4}\left(M_{1}^{2}, M_{1}^{2}, \tilde{m}_{R_{3}}^{2}, \tilde{m}_{R_{3}}^{2}\right)\right) \\
& \left.+\left(c_{R}^{2}-s_{R}^{2}\right)\left(J_{4}\left(M_{1}^{2}, M_{1}^{2}, \tilde{m}_{R_{2}}^{2}, \tilde{m}_{R_{3}}^{2}\right)+2 M_{1}^{2} I_{4}\left(M_{1}^{2}, M_{1}^{2}, \tilde{m}_{R_{2}}^{2}, \tilde{m}_{R_{3}}^{2}\right)\right)\right]
\end{aligned}
$$

\section{A.9 Contributions to $\Delta_{L, R}$}

The leading contributions to $\Delta_{L, R}$ are of two types: $\Delta_{L, R}=\Delta_{L, R}^{(b)}+\Delta_{L, R}^{(c)}$. The related diagrams are depicted in Fig. 32 and give:

$$
\begin{aligned}
\frac{\Delta_{L}^{(b)}}{s_{L} c_{L}} & =\frac{g^{2}}{16 \pi^{2}} \cdot \frac{3}{2} \mu M_{2} I_{3}\left(M_{2}^{2}, \mu^{2}, \tilde{m}_{L_{2}}^{2}\right)+\frac{g^{\prime 2}}{16 \pi^{2}} \cdot \frac{-1}{2} \mu M_{1} I_{3}\left(M_{1}^{2}, \mu^{2}, \tilde{m}_{L_{2}}^{2}\right) \\
& -\left(L_{2} \rightarrow L_{3}\right) \\
\frac{\Delta_{R}^{(b)}}{s_{R} c_{R}} & =\frac{g^{\prime 2}}{16 \pi^{2}} \mu M_{1} I_{3}\left(M_{1}^{2}, \mu^{2}, \tilde{m}_{R_{2}}^{2}\right)-\left(R_{2} \rightarrow R_{3}\right) \\
\frac{\Delta_{L}^{(c)}}{s_{L} c_{L}}= & \frac{g^{\prime 2}}{16 \pi^{2}} \mu M_{1}\left[-s_{R}^{2} I_{3}\left(M_{1}^{2}, \tilde{m}_{R_{2}}^{2}, \tilde{m}_{L_{2}}^{2}\right)-c_{R}^{2} I_{3}\left(M_{1}^{2}, \tilde{m}_{R_{3}}^{2}, \tilde{m}_{L_{2}}^{2}\right)\right]-\left(L_{2} \rightarrow L_{3}\right) \\
\frac{\Delta_{R}^{(c)}}{s_{R} c_{R}}= & \frac{g^{\prime 2}}{16 \pi^{2}} \mu M_{1}\left[-s_{L}^{2} I_{3}\left(M_{1}^{2}, \tilde{m}_{L_{2}}^{2}, \tilde{m}_{R_{2}}^{2}\right)-c_{L}^{2} I_{3}\left(M_{1}^{2}, \tilde{m}_{L_{3}}^{2}, \tilde{m}_{R_{2}}^{2}\right)\right]-\left(R_{2} \rightarrow R_{3}\right)
\end{aligned}
$$

\section{A.10 Contributions to muon $g-2$}

The MSSM contributions to the muon anomalous magnetic moment are described by diagrams analogous to those for $D^{\gamma}$ (Figs. 26, 27, 28), with the replacements $\tau\left(\tau^{c}\right) \rightarrow \mu\left(\mu^{c}\right)$. The result 


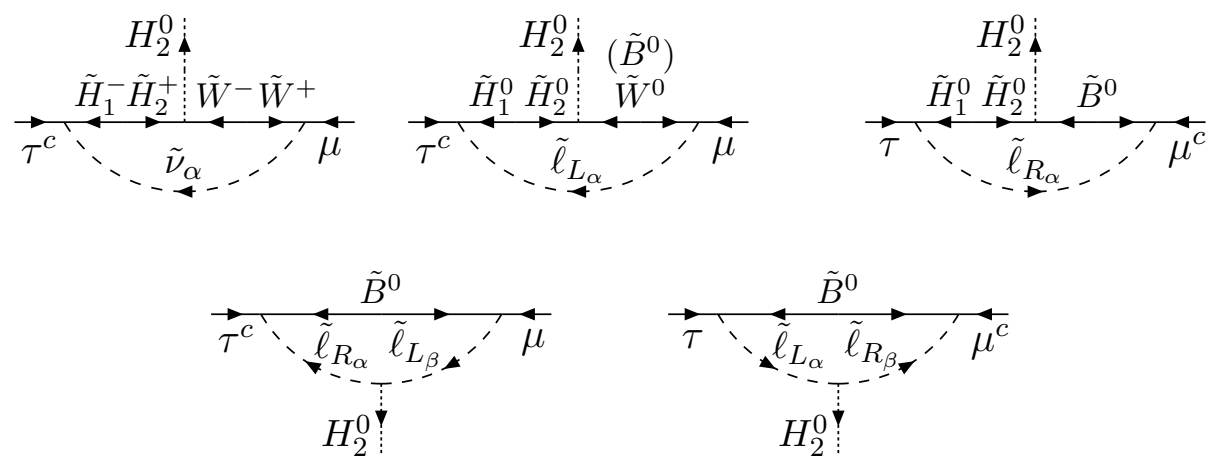

Figure 32: Diagrams that contribute to $\Delta_{L, R}^{(b)}$ (first row) and $\Delta_{L, R}^{(c)}$ (second row).

can be decomposed into five terms, i.e. $a_{\mu}^{\mathrm{MSSM}}=a_{\mu L}^{(a)}+a_{\mu R}^{(a)}+a_{\mu L}^{(b)}+a_{\mu R}^{(b)}+a_{\mu L R}^{(c)}$, given as follows:

$$
\begin{aligned}
& \frac{a_{\mu L}^{(a)}}{2 m_{\mu}^{2}}=\frac{g^{2}}{16 \pi^{2}} \cdot \frac{c_{L}^{2}}{4}\left[\tilde{m}_{L_{2}}^{2} J_{5}\left(M_{2}^{2}, \tilde{m}_{L_{2}}^{2}, \tilde{m}_{L_{2}}^{2}, \tilde{m}_{L_{2}}^{2}, \tilde{m}_{L_{2}}^{2}\right)-2 M_{2}^{2} J_{5}\left(M_{2}^{2}, M_{2}^{2}, M_{2}^{2}, M_{2}^{2}, \tilde{m}_{L_{2}}^{2}\right)\right] \\
& +\frac{g^{\prime 2}}{16 \pi^{2}} \cdot \frac{c_{L}^{2}}{4} \tilde{m}_{L_{2}}^{2} J_{5}\left(M_{1}^{2}, \tilde{m}_{L_{2}}^{2}, \tilde{m}_{L_{2}}^{2}, \tilde{m}_{L_{2}}^{2}, \tilde{m}_{L_{2}}^{2}\right)+\left(c_{L}^{2} \rightarrow s_{L}^{2}, L_{2} \rightarrow L_{3}\right) \\
& \frac{a_{\mu R}^{(a)}}{2 m_{\mu}^{2}}=\frac{g^{\prime 2}}{16 \pi^{2}} c_{R}^{2} \tilde{m}_{R_{2}}^{2} J_{5}\left(M_{1}^{2}, \tilde{m}_{R_{2}}^{2}, \tilde{m}_{R_{2}}^{2}, \tilde{m}_{R_{2}}^{2}, \tilde{m}_{R_{2}}^{2}\right)+\left(c_{R}^{2} \rightarrow s_{R}^{2}, R_{2} \rightarrow R_{3}\right) \\
& \frac{a_{\mu L}^{(b)}}{2 m_{\mu}^{2}}=\frac{g^{2}}{16 \pi^{2}} \cdot \frac{c_{L}^{2}}{2}\left[-\tilde{m}_{L_{2}}^{2} J_{5}\left(M_{2}^{2}, \mu^{2}, \tilde{m}_{L_{2}}^{2}, \tilde{m}_{L_{2}}^{2}, \tilde{m}_{L_{2}}^{2}\right)-2 \tilde{m}_{L_{2}}^{4} I_{5}\left(M_{2}^{2}, \mu^{2}, \tilde{m}_{L_{2}}^{2}, \tilde{m}_{L_{2}}^{2}, \tilde{m}_{L_{2}}^{2}\right)\right. \\
& +M_{2} \mu \tan \beta\left(\tilde{m}_{L_{2}}^{2} I_{5}\left(M_{2}^{2}, \mu^{2}, \tilde{m}_{L_{2}}^{2}, \tilde{m}_{L_{2}}^{2}, \tilde{m}_{L_{2}}^{2}\right)+2 J_{5}\left(M_{2}^{2}, M_{2}^{2}, \mu^{2}, \mu^{2}, \tilde{m}_{L_{2}}^{2}\right)\right. \\
& \left.\left.+2 J_{5}\left(M_{2}^{2}, M_{2}^{2}, M_{2}^{2}, \mu^{2}, \tilde{m}_{L_{2}}^{2}\right)+2 J_{5}\left(M_{2}^{2}, \mu^{2}, \mu^{2}, \mu^{2}, \tilde{m}_{L_{2}}^{2}\right)\right)\right] \\
& +\frac{g^{\prime 2}}{16 \pi^{2}} \frac{c_{L}^{2}}{2} \tilde{m}_{L_{2}}^{2}\left[J_{5}\left(M_{1}^{2}, \mu^{2}, \tilde{m}_{L_{2}}^{2}, \tilde{m}_{L_{2}}^{2}, \tilde{m}_{L_{2}}^{2}\right)-M_{1} \mu \tan \beta I_{5}\left(M_{1}^{2}, \mu^{2}, \tilde{m}_{L_{2}}^{2}, \tilde{m}_{L_{2}}^{2}, \tilde{m}_{L_{2}}^{2}\right)\right] \\
& +\left(c_{L}^{2} \rightarrow s_{L}^{2}, L_{2} \rightarrow L_{3}\right) \\
& \frac{a_{\mu R}^{(b)}}{2 m_{\mu}^{2}}=\frac{g^{\prime 2}}{16 \pi^{2}} c_{R}^{2} \tilde{m}_{R_{2}}^{2}\left[-J_{5}\left(M_{1}^{2}, \mu^{2}, \tilde{m}_{R_{2}}^{2}, \tilde{m}_{R_{2}}^{2}, \tilde{m}_{R_{2}}^{2}\right)+M_{1} \mu \tan \beta I_{5}\left(M_{1}^{2}, \mu^{2}, \tilde{m}_{R_{2}}^{2}, \tilde{m}_{R_{2}}^{2}, \tilde{m}_{R_{2}}^{2}\right)\right] \\
& +\left(c_{R}^{2} \rightarrow s_{R}^{2}, R_{2} \rightarrow R_{3}\right) \\
& \frac{a_{\mu L R}^{(c)}}{2 m_{\mu}^{2}}=-\frac{g^{\prime 2}}{16 \pi^{2}} M_{1}^{3} \times \\
& \left\{\left[\frac{m_{\tau}}{m_{\mu}}\left(c_{R}^{2} s_{L} c_{L} A_{\mu \tau}^{R}+s_{R} c_{R} c_{L}^{2} A_{\mu \tau}^{L}+s_{R} c_{R} s_{L} c_{L}\left[A_{\tau}+\mu \tan \beta\right]\right)\right.\right. \\
& \left.+c_{R}^{2} c_{L}^{2}\left(A_{\mu}+\mu \tan \beta\right)\right] I_{5}\left(M_{1}^{2}, M_{1}^{2}, M_{1}^{2}, \tilde{m}_{L_{2}}^{2}, \tilde{m}_{R_{2}}^{2}\right)
\end{aligned}
$$




$$
\begin{aligned}
& +\left[\frac{m_{\tau}}{m_{\mu}}\left(-c_{R}^{2} s_{L} c_{L} A_{\mu \tau}^{R}+s_{R} c_{R} s_{L}^{2} A_{\mu \tau}^{L}-s_{R} c_{R} s_{L} c_{L}\left[A_{\tau}+\mu \tan \beta\right]\right)\right. \\
& \left.+c_{R}^{2} s_{L}^{2}\left(A_{\mu}+\mu \tan \beta\right)\right] I_{5}\left(M_{1}^{2}, M_{1}^{2}, M_{1}^{2}, \tilde{m}_{L_{3}}^{2}, \tilde{m}_{R_{2}}^{2}\right) \\
& +\left[\frac{m_{\tau}}{m_{\mu}}\left(s_{R}^{2} s_{L} c_{L} A_{\mu \tau}^{R}-s_{R} c_{R} c_{L}^{2} A_{\mu \tau}^{L}-s_{R} c_{R} s_{L} c_{L}\left[A_{\tau}+\mu \tan \beta\right]\right)\right. \\
& \left.+s_{R}^{2} c_{L}^{2}\left(A_{\mu}+\mu \tan \beta\right)\right] I_{5}\left(M_{1}^{2}, M_{1}^{2}, M_{1}^{2}, \tilde{m}_{L_{2}}^{2}, \tilde{m}_{R_{3}}^{2}\right) \\
& +\left[\frac{m_{\tau}}{m_{\mu}}\left(-s_{R}^{2} s_{L} c_{L} A_{\mu \tau}^{R}-s_{R} c_{R} s_{L}^{2} A_{\mu \tau}^{L}+s_{R} c_{R} s_{L} c_{L}\left[A_{\tau}+\mu \tan \beta\right]\right)\right. \\
& \left.\left.+s_{R}^{2} s_{L}^{2}\left(A_{\mu}+\mu \tan \beta\right)\right] I_{5}\left(M_{1}^{2}, M_{1}^{2}, M_{1}^{2}, \tilde{m}_{L_{3}}^{2}, \tilde{m}_{R_{3}}^{2}\right)\right\}
\end{aligned}
$$

\section{References}

[1] J. Ellis and D. V. Nanopoulos, Phys. Lett. B 110 (1982) 44.

[2] J. S. Hagelin, S. Kelley and T. Tanaka, Nucl. Phys. B415 (1994) 293; F. Gabbiani, E. Gabrielli, A. Masiero and L. Silvestrini, Nucl. Phys. B 477 (1996) 321 [arXiv:hepph/9604387]; Y. G. Kim, P. Ko, J. S. Lee and K. Y. Lee, Phys. Rev. D 59 (1999) 055018 [arXiv:hep-ph/9811211]; I. Masina and C. A. Savoy, Nucl. Phys. B 661 (2003) 365 [arXiv:hep-ph/0211283].

[3] Y. Fukuda et al. [Super-Kamiokande Collaboration], Phys. Lett. B 433 (1998) 9 [arXiv:hepex/9803006] and Phys. Lett. B 436 (1998) 33 [arXiv:hep-ex/9805006]; J. A. Goodman [Super-Kamiokande Collaboration], Int. J. Mod. Phys. A 17 (2002) 3353; M. Ambrosio et al. [MACRO Collaboration], Phys. Lett. B 517 (2001) 59 [arXiv:hep-ex/0106049]; D. A. Petyt [SOUDAN-2 Collaboration], Nucl. Phys. Proc. Suppl. 110 (2002) 349.

[4] K. Abe et al. [Belle Collaboration], arXiv:hep-ex/0310029.

[5] Y. Yusa, H. Hayashii, T. Nagamine and A. Yamaguchi [Belle Collaboration], eConf C0209101 (2002) TU13 [Nucl. Phys. Proc. Suppl. 123 (2003) 95] [arXiv:hep-ex/0211017]; Y. Yusa et al. [Belle Collaboration], arXiv:hep-ex/0403039.

[6] B. Aubert et al. [BABAR Collaboration], Phys. Rev. Lett. 92 (2004) 121801 [arXiv:hepex/0312027].

[7] K. Hagiwara et al. [Particle Data Group Collaboration], Phys. Rev. D 66 (2002) 010001.

[8] Y. Enari et al. [Belle Collaboration], arXiv:hep-ex/0404018. 
[9] SuperKEKB, Letter of intent, available at http//Belle.kek.jp/superb.

[10] J. A. Aguilar-Saavedra et al. [ECFA/DESY LC Physics Working Group Collaboration], arXiv:hep-ph/0106315.

[11] A. Brignole and A. Rossi, Phys. Lett. B 566 (2003) 217 [arXiv:hep-ph/0304081].

[12] C. J. C. Burges and H. J. Schnitzer, Nucl. Phys. B 228 (1983) 464; W. Buchmuller and D. Wyler, Nucl. Phys. B 268 (1986) 621.

[13] S. Nussinov, R. D. Peccei and X. M. Zhang, Phys. Rev. D 63 (2001) 016003 [arXiv:hepph/0004153]; D. Delepine and F. Vissani, Phys. Lett. B 522 (2001) 95 [arXiv:hepph/0106287]; A. Flores-Tlalpa, J. M. Hernandez, G. Tavares-Velasco and J. J. Toscano, Phys. Rev. D 65 (2002) 073010 [arXiv:hep-ph/0112065].

[14] D. Black, T. Han, H. J. He and M. Sher, Phys. Rev. D 66 (2002) 053002 [arXiv:hepph/0206056]; M. Sher and I. Turan, Phys. Rev. D 69 (2004) 017302 [arXiv:hepph/0309183]; S. N. Gninenko, M. M. Kirsanov, N. V. Krasnikov and V. A. Matveev, Mod. Phys. Lett. A 17 (2002) 1407 [arXiv:hep-ph/0106302].

[15] R. Kaiser and H. Leutwyler, arXiv:hep-ph/9806336.

[16] T. Feldmann, Int. J. Mod. Phys. A 15 (2000) 159 [arXiv:hep-ph/9907491].

[17] A. Brignole and A. Rossi, Nucl. Phys. B 587 (2000) 3 [arXiv:hep-ph/0006036].

[18] L. J. Hall, V. A. Kostelecky and S. Raby, Nucl. Phys. B 267 (1986) 415.

[19] J. Hisano, T. Moroi, K. Tobe and M. Yamaguchi, Phys. Rev. D 53 (1996) 2442 [arXiv:hep-ph/9510309] and Phys. Lett. B 391 (1997) 341 [Erratum-ibid. B 397 (1997) 357] [arXiv:hep-ph/9605296]; J. Hisano, D. Nomura and T. Yanagida, Phys. Lett. B 437 (1998) 351 [arXiv:hep-ph/9711348]; J. Hisano and D. Nomura, Phys. Rev. D 59 (1999) 116005 [arXiv:hep-ph/9810479].

[20] K. S. Babu and C. Kolda, Phys. Rev. Lett. 89 (2002) 241802 [arXiv:hep-ph/0206310]; A. Dedes, J. R. Ellis and M. Raidal, Phys. Lett. B 549 (2002) 159 [arXiv:hep-ph/0209207].

[21] M. Sher, Phys. Rev. D 66 (2002) 057301 [arXiv:hep-ph/0207136].

[22] J. L. Diaz-Cruz and J. J. Toscano, Phys. Rev. D 62 (2000) 116005 [arXiv:hep-ph/9910233]; M. Sher, Phys. Lett. B 487 (2000) 151 [arXiv:hep-ph/0006159]; T. Han and D. Marfatia, Phys. Rev. Lett. 86 (2001) 1442 [arXiv:hep-ph/0008141]; U. Cotti, L. Diaz-Cruz, C. Pagliarone and E. Vataga, arXiv:hep-ph/0111236; K. A. Assamagan, A. Deandrea and P. A. Delsart, Phys. Rev. D 67 (2003) 035001 [arXiv:hep-ph/0207302]. 
[23] L. J. Hall, R. Rattazzi and U. Sarid, Phys. Rev. D 50 (1994) 7048 [arXiv:hep-ph/9306309]; R. Hempfling, Phys. Rev. D 49 (1994) 6168; M. Carena, M. Olechowski, S. Pokorski and C. E. Wagner, Nucl. Phys. B 426 (1994) 269 [arXiv:hep-ph/9402253]; D. M. Pierce, J. A. Bagger, K. T. Matchev and R. j. Zhang, Nucl. Phys. B 491 (1997) 3 [arXiv:hep$\mathrm{ph} / 9606211]$.

[24] M. Carena, S. Mrenna and C. E. Wagner, Phys. Rev. D 60 (1999) 075010 [arXiv:hepph/9808312] and Phys. Rev. D 62 (2000) 055008 [arXiv:hep-ph/9907422].

[25] D. J. Gross, S. B. Treiman and F. Wilczek, Phys. Rev. D 19 (1979) 2188; V. A. Novikov, M. A. Shifman and A. I. Vainshtein, Nucl. Phys. B 165 (1980) 55.

[26] P. Fayet, CERN-TH-2864 Contributed to Europhysics Study Conf. on Unification of Fundamental Interactons, Erice, Italy, Mar 17-24, 1980; J. A. Grifols and A. Mendez, Phys. Rev. D 26 (1982) 1809; J. R. Ellis, J. S. Hagelin and D. V. Nanopoulos, Phys. Lett. B 116 (1982) 283; R. Barbieri and L. Maiani, Phys. Lett. B 117 (1982) 203; D. A. Kosower, L. M. Krauss and N. Sakai, Phys. Lett. B 133 (1983) 305; T. C. Yuan, R. Arnowitt, A. H. Chamseddine and P. Nath, Z. Phys. C 26 (1984) 407; J. C. Romao, A. Barroso, M. C. Bento and G. C. Branco, Nucl. Phys. B 250 (1985) 295; J. L. Lopez, D. V. Nanopoulos and X. Wang, Phys. Rev. D 49 (1994) 366 [arXiv:hep-ph/9308336]; U. Chattopadhyay and P. Nath, Phys. Rev. D 53 (1996) 1648.

[27] T. Moroi, Phys. Rev. D 53 (1996) 6565 [Erratum-ibid. D 56 (1997) 4424] [arXiv:hep$\mathrm{ph} / 9512396]$.

[28] M. Graesser and S. Thomas, Phys. Rev. D 65 (2002) 075012 [arXiv:hep-ph/0104254]; J. L. Feng, K. T. Matchev and Y. Shadmi, Nucl. Phys. B 613 (2001) 366 [arXiv:hepph/0107182]; A. Romanino and A. Strumia, Nucl. Phys. B 622 (2002) 73 [arXiv:hepph/0108275]; G. C. Cho, N. Haba and J. Hisano, Phys. Lett. B 529 (2002) 117 [arXiv:hepph/0112163]; S. Baek, P. Ko and J. H. Park, Eur. Phys. J. C 24 (2002) 613 [arXiv:hep$\mathrm{ph} / 0203251]$.

[29] M. Davier, S. Eidelman, A. Hocker and Z. Zhang, Eur. Phys. J. C 31 (2003) 503 [arXiv:hepph/0308213]; K. Melnikov and A. Vainshtein, arXiv:hep-ph/0312226.

[30] S. P. Martin and J. D. Wells, Phys. Rev. D 67 (2003) 015002 [arXiv:hep-ph/0209309].

[31] LEPSUSYWG, ALEPH, DELPHI, L3 and OPAL experiments (http://lepsusy.web.cern.ch/lepsusy/Welcome.html).

[32] J. Abdallah et al. [DELPHI Collaboration], Eur. Phys. J. C 31 (2004) 421 [arXiv:hepex/0311019].

[33] M. J. S. Levine, Phys. Rev. D 36 (1987) 1329; F. Gabbiani, J. H. Kim and A. Masiero, Phys. Lett. B 214 (1988) 398.

[34] J. I. Illana and M. Masip, Phys. Rev. D 67 (2003) 035004 [arXiv:hep-ph/0207328]. 
[35] M. L. Brooks et al. [MEGA Collaboration], Phys. Rev. Lett. 83 (1999) 1521 [arXiv:hepex/9905013].

[36] F. Borzumati and A. Masiero, Phys. Rev. Lett. 57 (1986) 961.

[37] R. Barbieri and L. J. Hall, Phys. Lett. B 338 (1994) 212 [arXiv:hep-ph/9408406]; R. Barbieri, L. J. Hall and A. Strumia, Nucl. Phys. B 445 (1995) 219 [arXiv:hepph/9501334]; J. Hisano, T. Moroi, K. Tobe, M. Yamaguchi and T. Yanagida, Phys. Lett. B 357 (1995) 579 [arXiv:hep-ph/9501407]; J. L. Feng, Y. Nir and Y. Shadmi, Phys. Rev. D 61 (2000) 113005 [arXiv:hep-ph/9911370]; J. R. Ellis, M. E. Gomez, G. K. Leontaris, S. Lola and D. V. Nanopoulos, Eur. Phys. J. C 14 (2000) 319 [arXiv:hepph/9911459]; W. Buchmuller, D. Delepine and L. T. Handoko, Nucl. Phys. B 576 (2000) 445 [arXiv:hep-ph/9912317]; D. F. Carvalho, M. E. Gomez and S. Khalil, JHEP 0107 (2001) 001 [arXiv:hep-ph/0101250]; J. A. Casas and A. Ibarra, Nucl. Phys. B 618 (2001) 171 [arXiv:hep-ph/0103065]; T. Blazek and S. F. King, Phys. Lett. B 518 (2001) 109 [arXiv:hep-ph/0105005]; S. Lavignac, I. Masina and C. A. Savoy, Phys. Lett. B 520 (2001) 269 [arXiv:hep-ph/0106245]; A. Rossi, Phys. Rev. D 66 (2002) 075003 [arXiv:hepph/0207006]; A. Masiero, S. K. Vempati and O. Vives, Nucl. Phys. B 649 (2003) 189 [arXiv:hep-ph/0209303]; K. S. Babu, B. Dutta and R. N. Mohapatra, Phys. Rev. D 67 (2003) 076006 [arXiv:hep-ph/0211068]; T. Blazek and S. F. King, Nucl. Phys. B 662 (2003) 359 [arXiv:hep-ph/0211368]; A. Masiero, S. Profumo, S. K. Vempati and C. E. Yaguna, JHEP 0403 (2004) 046 [arXiv:hep-ph/0401138].

[38] J. Hisano, M. M. Nojiri, Y. Shimizu and M. Tanaka, Phys. Rev. D 60 (1999) 055008 [arXiv:hep-ph/9808410]; I. Hinchliffe and F. E. Paige, Phys. Rev. D 63 (2001) 115006 [arXiv:hep-ph/0010086]; M. Guchait, J. Kalinowski and P. Roy, Eur. Phys. J. C 21 (2001) 163 [arXiv:hep-ph/0103161]; W. Porod and W. Majerotto, Phys. Rev. D 66 (2002) 015003 [arXiv:hep-ph/0201284]; D. F. Carvalho, J. R. Ellis, M. E. Gomez, S. Lola and J. C. Romao, arXiv:hep-ph/0206148; F. Deppisch, H. Pas, A. Redelbach, R. Ruckl and Y. Shimizu, Phys. Rev. D 69 (2004) 054014 [arXiv:hep-ph/0310053]; F. Deppisch, J. Kalinowski, H. Pas, A. Redelbach and R. Ruckl, arXiv:hep-ph/0401243. 